\title{
Estimação de Modelos de Markov Ocultos Usando Aritmética Intervalar
}

\author{
Tiago de Morais Montanher \\ TESE APRESENTADA \\ $\mathrm{AO}$ \\ Instituto de Matemática e Estatística \\ DA \\ Universidade DE SÃo PAUlo \\ PARA \\ OBTENÇÃO DO TÍTULO \\ $\mathrm{DE}$ \\ DOUTOR EM CIÊNCIAS \\ Programa: Matemática Aplicada \\ Orientador: Prof. Dr. Walter F. Mascarenhas
}

Durante o desenvolvimento deste trabalho o autor recebeu auxílio financeiro da CAPES e CNPq.

O autor também recebeu auxílio do projeto 2013/10916-2 da FAPESP na forma de recursos

computacionais para a realização de experimentos

São Paulo, fevereiro de 2015 


\title{
Estimação de Modelos de Markov Ocultos Usando Aritmética Intervalar
}

\author{
Esta versão da tese contém as correções e alterações sugeridas \\ pela Comissão Julgadora durante a defesa da versão original do trabalho, \\ realizada em 24/04/2015. Uma cópia da versão original estão disponível no \\ Instituto de Matemática e EstatÃstica da Universidade de Sã£o Paulo.
}

Comissão Julgadora:

- Prof. Dr. Walter F. Mascarenhas (orientador) - IME-USP

- Prof. Dr. Julio M. Stern - IME-USP

- Profa. Dra. Chang C. Yu Dorea - UNB

- Prof. Dr. José M. Martinez - UNICAMP

- Prof. Dr. Fabio G. Cozman 


\section{Agradecimentos}

Uma tese de doutorado é um trabalho realizado por muitas mãos. Isso leva a um número grande de agradecimentos, o que aumenta a probabilidade de esquecermos algum nome importante. Em todo caso, vou tentar minimizar globalmente a quantidade de pessoas esquecidas.

Agradeço inicialmente ao meu orientador, Walter Mascarenhas. Ao longo dos anos ele buscou estimular minha curiosidade e me deu conselhos valiosos. Há vários colegas e amigos no IME que participaram ativamente deste trabalho. Dentre eles, destaco o Pedro Peixoto e o Rafael Lobato. Eles contribuíram com críticas e sugestões para os experimentos e análises realizadas. Em particular o Rafael Lobato me ajudou com os gráficos do capítulo 5.

Além deles, foi bastante importante a convivência com os colegas dos laboratórios de matemática aplicada, otimização e do grupo de pesquisa do Walter. Agradeço ao John Gardenghi, Ademar Lacerda, André Euler, Vitor, Antonio Marcos e Rafael Schimidt. Agradeço aos professores Ernesto Birgin e Paulo Silva que participaram do meu exame de qualificação e contribuíram com várias sugestões. Finalmente agradeço aos membros da banca julgadora pelas valiosas considerações e apontamentos sobre o trabalho.

Durante dois anos tive a oportunidade de trabalhar para a Petrobras. Nesse período conheci ótimos profissionais e amigos. Agradeço ao Daniel Trovó, Daniel Ferber e Daniel Alfenas, Ricardo Paixão e Thiago Silva. Além deles, tive o privilégio e prazer de trabalhar com o Fernando Marcellino e o Fernando Freire. Certamente essas pessoas são mais do que colegas de trabalho e tenho um carinho muito grande por todos.

Meus amigos do chá e shandalar tornaram esses anos de doutorado muito mais divertidos. A todos eles meu muito obrigado e a certeza de que essa é só mais uma etapa que passamos juntos. Além deles, agradeço à Giselle Bertaggia pela amizade de vários anos.

Agradeço à minha família por todo suporte e paciência. Meus pais, Sonia e Antonio que sempre me estimularam a seguir no caminho da ciência e meu irmão Danilo, que sempre traz bons questionamentos sobre a vida. Certamente eles tem muito a ver com o sucesso desse trabalho.

Finalmente, agradeço à Bianca Paoletti pelo amor e dedicação que teve comigo desde que nos conhecemos. Certamente o fim desse trabalho é só o começo de uma nova vida.

Essa conquista é de todas as pessoas que citei aqui e de outras que passaram na minha vida, contribuindo de alguma forma para que eu me tornasse um matemático melhor. A todas essas pessoas, obrigado. 


\section{Resumo}

MONTANHER, T. M. Estimação de modelos de Markov ocultos usando aritmética inter-

valar. 2015. Tese (Doutorado) - Instituto de Matemática e Estatística, Universidade de São Paulo, São Paulo, 2015.

Modelos de Markov ocultos (MMOs) são uma ferramenta importante em matemática aplicada e estatística. Eles se baseiam em dois processos estocásticos. O primeiro é uma cadeia de Markov, que não é observada diretamente. O segundo é observável e sua distribuição depende do estado na cadeia de Markov. Supomos que os processos são discretos no tempo e assumem um número finito de estados. Para extrair informações dos MMOs, é necessário estimar seus parâmetros. Diversos algoritmos locais têm sido utilizados nas últimas décadas para essa tarefa. Nosso trabalho estuda a estimação de parâmetros em modelos de Markov ocultos, do ponto de vista da otimização global. Desenvolvemos algoritmos capazes de encontrar, em uma execução bem sucedida, todos os estimadores de máxima verossimilhança globais de um modelo de Markov oculto. Para tanto, usamos aritmética intervalar. Essa aritmética permite explorar sistematicamente o espaço paramétrico, excluindo regiões que não contém soluções. O cálculo da função objetivo é feito através da recursão backward, descrita na literatura estatística. Modificamos a extensão intervalar natural dessa recursão usando programação linear. Nossa abordagem é mais eficiente e produz intervalos mais estreitos do que a implementação padrão. Experimentos mostram ganhos de 16 a 250 vezes, de acordo com a complexidade do modelo. Revisamos os algoritmos locais, tendo em vista sua aplicação em métodos globais. Comparamos os algoritmos de Baum-Welch, pontos interiores e gradientes projetados espectrais. Concluímos que o método de Baum-Welch é o mais indicado como auxiliar em otimização global. Modificamos o interval branch and bound para resolver a estimação de modelos com eficiência. Usamos as condições KKT e as simetrias do problema na construção de testes para reduzir ou excluir caixas. Implementamos procedimentos de aceleração da convergência, como o método de Newton intervalar e propagação de restrições e da função objetivo. Nosso algoritmo foi escrito em $C++$, usando programação genérica. Mostramos que nossa implementação dá resultados tão bons quanto o resolvedor global BARON, porém com mais eficiência. Em média, nosso algoritmo é capaz de resolver $50 \%$ mais problemas no mesmo período de tempo. Concluímos estudando aspectos qualitativos dos MMOs com mistura Bernoulli. Plotamos todos os máximos globais detectados em instâncias com poucas observações e apresentamos novos limitantes superiores da verossimilhança baseados na divisão de uma amostra grande em grupos menores.

Palavras-chave: Modelos de Markov Ocultos, Aritmética Intervalar, Otimização Global. 


\section{Abstract}

MONTANHER, T. M. Estimating Hidden Markov Model Parameters Using Interval Arithmetic. 2015. PhD Thesis - Instituto de Matemática e Estatística, Universidade de São Paulo, São Paulo, 2015.

Hidden Markov models(HMMs) are an important tool in statistics and applied mathematics. Our work deals with processes formed by two discrete time and finite state space stochastic processes. The first process is a Markov chain and is not directly observed. On the other hand, the second process is observable and its distribution depends on the current state of the hidden component. In order to extract conclusions from a Hidden Markov Model we must estimate the parameters that defines it. Several local algorithms has been used to handle with this task. We present a global optimization approach based on interval arithmetic to maximize the likelihood function. Interval arithmetic allow us to explore parametric space systematically, discarding regions which cannot contain global maxima. We evaluate the objective function and its derivatives by the so called backward recursion and show that is possible to obtain sharper interval extensions for such functions using linear programming. Numerical experiments shows that our approach is 16 to 250 times more efficient than standard implementations. We also study local optimization algorithms hidden Markov model estimation. We compare Baum-Welch procedure with interior points and spectral projected gradients. We conclude that Baum-Welch is the best option as a sub-algorithm in a global optimization framework. We improve the well known interval branch and bound algorithm to take advantages on the problem structure. We derive new exclusion tests, based on its KKT conditions and symmetries. We implement our approach in $\mathrm{C}++$, under generic programming paradigm. We show that our implementation is compatible with global optimization solver BARON in terms of precision. We also show that our algorithm is faster than BARON. In average, we can handle with $50 \%$ more problems within the same amount of time. We conclude studying qualitative aspects of Bernoulli hidden Markov models. We plot all global maxima found in small observations instances and show a new upper bound of the likelihood based on splitting observations in small groups.

Keywords: Hidden Markov Models, Interval Arithmetic, Global Optimization. 


\section{Sumário}

$\begin{array}{ll}\text { Lista de Abreviaturas } & \text { ix }\end{array}$

Lista de Símbolos $\quad$ xi

Lista de Figuras $\quad$ xiii

Lista de Tabelas $\quad$ xvii

1 Introdução 1

2 Aritmética Intervalar $\quad \mathbf{5}$

2.1 Conceitos fundamentais $\ldots \ldots \ldots \ldots \ldots \ldots \ldots$

2.1 .1 Operações Fundamentais . . . . . . . . . . . . . . . . . . 6

2.1 .2 Propriedade de Inclusão ～. . . . . . . . . . . . . . . . . . . . . 7

2.1 .3 Teorema do Valor Médio Intervalar . . . . . . . . . . . . . . . . . . . 8

2.2 Aspectos Computacionais . . . . . . . . . . . . . . . . . 9

2.2.1 Modo de Arredondamento e operações elementares . . . . . . . . . . . . . . . 9

2.2 .2 Divisão estendida . . . . . . . . . . . . . . . . . . . . 10

2.3 Sistemas Lineares Intervalares . . . . . . . . . . . . . . . . . . . . 11

2.3 .1 Gauss-Seidel Intervalar . . . . . . . . . . . . . . . . . . . . . 12

2.3 .2 Pré-Condicionamento . . . . . . . . . . . . . . . . . . 12

2.4 Exemplo - Otimização Global . . . . . . . . . . . . . . . . . 13

3 Modelos de Markov Ocultos 15

3.1 Modelos de Mistura . . . . . . . . . . . . . . . . . . . . . . . . . 15

3.2 Cadeias de Markov . . . . . . . . . . . . . . . . . . . . 17

3.3 Modelos de Markov Ocultos . . . . . . . . . . . . . . . . . . . . . . . . . 19

3.3 .1 Definição . . . . . . . . . . . . . . . . . . . . . . 19

3.3.2 Modelos de Markov Ocultos Intervalares . . . . . . . . . . . . . . . . . . . 19

3.4 Distribuição Invariante Intervalar ～. . . . . . . . . . . . . . . . . 20

4 Avaliando a Verossimilhança $\quad 23$

4.1 Revisão do Caso Real . . . . . . . . . . . . . . . . . . . . . . . . . 24

4.1 .1 Recursões Forward-Backward . . . . . . . . . . . . . . . 24

4.1 .2 Normalização . . . . . . . . . . . . . . . . . . . . . 25

4.1 .3 Função de Verossimilhança . . . . . . . . . . . . . . . . . . . 28 
4.2 Extensão Natural . . . . . . . . . . . . . . . . . . . . . . . . 30

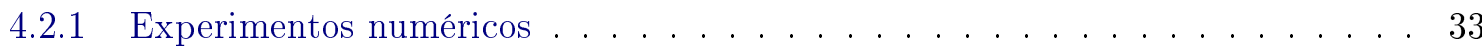

4.3 Extensão com Programação Linear . . . . . . . . . . . . . . . . . . 37

4.3 .1 Experimentos Numéricos . . . . . . . . . . . . . . . . . . . . . 39

5 Estimando Parâmetros $\quad 43$

5.1 Condições KKT . . . . . . . . . . . . . . . . . . . . . . . . . . . . . . . . 44

5.2 Estimação Local dos Parâmetros . . . . . . . . . . . . . . . . . . . . . 46

5.2 .1 Baum-Welch . . . . . . . . . . . . . . . . . . . . 47

5.2 .2 Gradientes Projetados Espectrais - SPG . . . . . . . . . . . . . . 48

5.3 Experimentos Numéricos dos Métodos Locais . . . . . . . . . . . . . . . . . . 50

5.3 .1 Análise dos Métodos . . . . . . . . . . . . . . . . . 50

5.3 .2 Detectando Máximos Globais . . . . . . . . . . . . . . . . . . . . 53

5.4 O Algoritmo de Branch and Bound Intervalar . . . . . . . . . . . . . . . 60

5.4 .1 Exclusão e Redução de Caixas . . . . . . . . . . . . . . . . . . . . . 62

5.5 Quebra de Simetria . . . . . . . . . . . . . . . . . . . . 63

5.6 Aceleração da Convergência . . . . . . . . . . . . . . . . . . . . 65

5.6 .1 Método de Newton . . . . . . . . . . . . . . . . . . . . 65

5.6 .2 Propagação da Função Objetivo . . . . . . . . . . . . . . . . . . . . . 66

5.7 Experimentos Numéricos . . . . . . . . . . . . . . . . . . . 6 67

5.8 Experimentos Numéricos . . . . . . . . . . . . . . . . . . . . . 77

6 Conclusões $\quad 85$

$\begin{array}{ll}\text { A Pseudo-Código dos Métodos Intervalares } & 87\end{array}$

$\begin{array}{ll}\text { Referências Bibliográficas } & 91\end{array}$

$\begin{array}{ll}\text { Índice Remissivo } & 95\end{array}$ 


\title{
Lista de Abreviaturas
}

\author{
MMO Modelo de Markov Oculto (Hidden Markov Model). \\ $M M O_{N, M} \quad$ Modelo de Markov Oculto com $N$ estados ocultos e $M$ símbolos \\ $\mathrm{B} \& \mathrm{~B} \quad$ Branch and Bound (Branch and Bound). \\ NaN Not a Number. \\ IEEE754 Padrão dos números de ponto flutuante.
}




\title{
Lista de Símbolos
}

\author{
$(A, \pi) \quad$ Cadeia de Markov. \\ $\theta=(A, B, \pi) \quad$ Modelo de Markov oculto. \\ $A \quad$ Matriz de transição. \\ $\pi \quad$ Vetor de distribuição inicial. \\ $B \quad$ Matriz de emissão. \\ $N \quad$ Número de estados na cadeia oculta. \\ $M \quad$ Emissões no modelo de mistura. \\ $O=\left(o_{1} \ldots o_{T}\right) \quad$ Conjunto de observações. \\ $q=\left(q_{1} \ldots q_{T}\right) \quad$ Estados ocultos associados a um conjunto de observações. \\ $A^{\prime} \quad$ Transposta da matriz $A$. \\ $A_{i} \quad$ Linha $i$ da matriz $A$. \\ $\boldsymbol{x} \quad$ Intervalo ou vetor interval. \\ $\boldsymbol{f} \quad$ Extensão intervalar da função $f$. \\ $\boldsymbol{A} \quad$ Matriz intervalar. \\ $\boldsymbol{\theta}=(\boldsymbol{A}, \boldsymbol{B}, \boldsymbol{\pi}) \quad$ Modelo de Markov oculto intervalar. \\ $L_{O}(\theta)=L(\theta) \quad$ Função de verossimilhança. \\ $\nabla($.$) \quad Operação realizada com o modo de arredondamento para baixo.$ \\ $\triangle($.) $\quad$ Operação realizada com o modo de arredondamento para cima. \\ $\mathcal{S} \quad$ Conjunto de todas as soluções do sistema linear intervalar $\boldsymbol{A x}=\boldsymbol{b}$.
}




\section{Lista de Figuras}

1.1 Representação de um modelo de Markov oculto . . . . . . . . . . . . . . . . . 1

1.2 Gráfico de $x^{3}+3 x^{2}+2$ no intervalo $[-1,2] \ldots \ldots \ldots \ldots \ldots$

2.1 Representação dos diferentes modos de arredondamento. . . . . . . . . . . . . . . 9

2.2 Solução de (2.15) obtida com a função plotlinsol do INTLAB. Em vermelho o conjunto $\mathcal{S}$ e tracejado em azul seu casco intervalar. . . . . . . . . . . . . . . 11

2.3 Exemplo do interval branch and bound - Início do Algoritmo . . . . . . . . . . . . . 13

2.4 Exemplo do interval branch and bound - Primeira iteração . . . . . . . . . . . . . . 14

2.5 Exemplo do interval branch and bound - Quarta iteração . . . . . . . . . . . . . . . 14

3.1 Realizações de um modelo de Markov oculto, com mistura normal. As componentes da mistura são $X_{1} \sim N(5,10)$ e $X_{2} \sim N(25,5)$. Na esquerda temos o componente escolhido a cada iteração, no meio sua função densidade e à direita o valor observado. 16

3.2 Comparação entre os algoritmos Gauss-Seidel intervalar e verifylss para limitar a

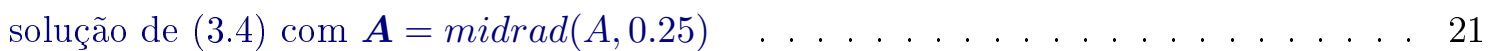

4.1 Cálculo do logaritmo realizado com a função padrão da cmath.h e com a biblioteca MPFR, especificando o modo de arredondamento para baixo. Amostras geradas aleatoriamente no intervalo $[0,1] \ldots \ldots \ldots \ldots \ldots \ldots$

4.2 Tempo necessário para a avaliação de $\mathbb{P}(O \mid \theta)$ em amostras de tamanho $T$, dadas na tabela anterior. A linha azul se refere à nossa estratégia de normalização. A linha vermelha nos dá o tempo da normalização com log . . . . . . . . . . . . . . . 28

4.3 Comparação entre nossa modificação da extensão natural e a forma padrão no cálculo de $\boldsymbol{L}(\boldsymbol{\theta}) \ldots \ldots \ldots \ldots \ldots$

4.4 Comparação entre nossa modificação da extensão natural e a forma padrão no cálculo

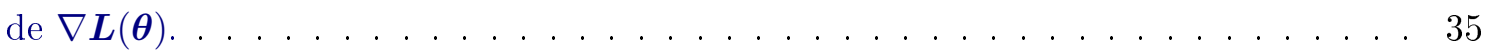

4.5 Comparação entre nossa modificação da extensão natural e a forma padrão no cálculo

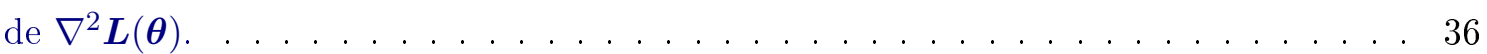

4.6 Comparação entre os limitantes superiores de $\boldsymbol{L}_{P L}(\boldsymbol{\theta})$ e $\boldsymbol{L}_{N T}(\boldsymbol{\theta})$. Os parâmetros dos modelos têm raio $0.001 \ldots \ldots \ldots$. . . . . . . . . . . . . . 40

4.7 Comparação entre os limitantes superiores de $\boldsymbol{L}_{P L}(\boldsymbol{\theta})$ e $\boldsymbol{L}_{N T}(\boldsymbol{\theta})$. Os parâmetros dos

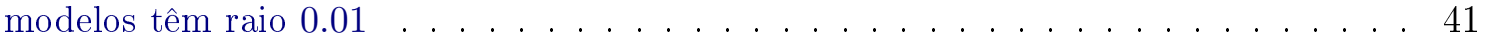

4.8 Comparação entre os limitantes superiores de $\boldsymbol{L}_{P L}(\boldsymbol{\theta})$ e $\boldsymbol{L}_{N T}(\boldsymbol{\theta})$. Os parâmetros dos

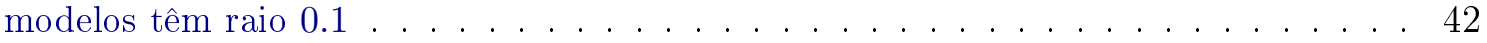


5.1 Comparação entre Baum-Welch, SPG e pontos interiores. Todas as instâncias possíveis do $M M O_{2,2}$ com $T=10, \ldots, 15$. A esquerda temos o máximo. A direita, o tempo necessário para obter a solução em segundos. Na primeira linha temos os

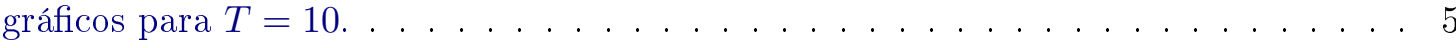

5.2 Comparação entre Baum-Welch, SPG e pontos interiores. Instâncias geradas aleatoriamente $M M O_{2,2}$ com $T=50,60, \ldots, 100$. A esquerda temos o máximo. A direita, o tempo necessário para obter a solução em segundos. Na primeira linha temos os gráficos para $T=50 \ldots \ldots \ldots \ldots \ldots \ldots \ldots \ldots \ldots$

5.3 Porcentagem de problemas em que cada método encontrou a melhor solução. Todas as instâncias possíveis do $M M O_{2,2} \operatorname{com} T=10, \ldots, 15 \ldots \ldots \ldots \ldots$. . . . . 53

5.4 Porcentagem de problemas em que cada método encontrou a melhor solução. Resultado de 100 instâncias do $M M O_{2,2}$ geradas aleatoriamente com $T=50,60, \ldots, 100$.

5.5 Dispersão das soluções obtidas com métodos locais, a partir de 50 pontos iniciais distintos. Nos dois primeiros gráficos, temos os estimadores do $M M O_{2,2}$ com amostra de tamanho 40, gerada a partir do modelo (5.39). O máximo global, obtido com nosso algoritmo e o modelo original também estão representados. O terceiro gráfico nos dá o máximo obtido por cada método local ao longo das iterações. . . . . . . . . . . . . 5

5.6 Dispersão das soluções obtidas com métodos locais, a partir de 50 pontos iniciais distintos. Nos dois primeiros gráficos, temos os estimadores do $M M_{2,2}$ com amostra de tamanho 60, gerada a partir do modelo (5.39). O máximo global, obtido com nosso algoritmo e o modelo original também estão representados. O terceiro gráfico nos dá o máximo obtido por cada método local ao longo das iterações. . . . . . . . . . . . .

5.7 Dispersão das soluções obtidas com métodos locais, a partir de 50 pontos iniciais distintos. Nos dois primeiros gráficos, temos os estimadores do $M M O_{2,2}$ com amostra de tamanho 90, gerada a partir do modelo (5.39). O máximo global, obtido com nosso algoritmo e o modelo original também estão representados. O terceiro gráfico nos dá o máximo obtido por cada método local ao longo das iterações. . . . . . . . . . . . .

5.8 Dispersão das soluções obtidas com métodos locais, a partir de 50 pontos iniciais distintos. Nos dois primeiros gráficos, temos os estimadores do $M M_{2,2}$ com amostra de tamanho 40, gerada a partir do modelo (5.40). O máximo global, obtido com nosso algoritmo e o modelo original também estão representados. O terceiro gráfico nos dá o máximo obtido por cada método local ao longo das iterações. . . . . . . . . . . . . 5

5.9 Dispersão das soluções obtidas com métodos locais, a partir de 50 pontos iniciais distintos. Nos dois primeiros gráficos, temos os estimadores do $M M O_{2,2}$ com amostra de tamanho 60, gerada a partir do modelo (5.40). O máximo global, obtido com nosso algoritmo e o modelo original também estão representados. O terceiro gráfico nos dá o máximo obtido por cada método local ao longo das iterações. . . . . . . . . . . . .

5.10 Dispersão das soluções obtidas com métodos locais, a partir de 50 pontos iniciais distintos. Nos dois primeiros gráficos, temos os estimadores do $M M O_{2,2}$ com amostra de tamanho 90, gerada a partir do modelo (5.40). O máximo global, obtido com nosso algoritmo e o modelo original também estão representados. O terceiro gráfico nos dá o máximo obtido por cada método local ao longo das iterações. . . . . . . . . . . . . 
5.11 Comparação entre BARON, HMM SOLVER com Baum-Welch e HMM SOLVER com SPG. Teste realizado com o $M M O_{2,2}$. Todas as instâncias com 5 observações. . 68

5.12 Comparação entre BARON, HMM SOLVER com Baum-Welch e HMM SOLVER com SPG. Teste realizado com o $M M O_{2,2}$. Todas as instâncias com 6 observações. . 69

5.13 Comparação entre BARON, HMM SOLVER com Baum-Welch e HMM SOLVER com SPG. Teste realizado com o $M M_{2,2}$. Todas as instâncias com 7 observações. . 70

5.14 Comparação entre BARON, HMM SOLVER com Baum-Welch e HMM SOLVER com SPG. Teste realizado com o $M M O_{2,2}$. Todas as instâncias com 8 observações. . 71

5.15 Comparação entre BARON, HMM SOLVER com Baum-Welch e HMM SOLVER com SPG. Teste realizado com o $M M_{2,2}$. Todas as instâncias com 9 observações. . 72

5.16 Comparação entre BARON, HMM SOLVER com Baum-Welch e HMM SOLVER com SPG. Teste realizado com o $M M O_{2,2}$. Todas as instâncias com 10 observações. . 73

5.17 Comparação entre BARON, HMM SOLVER com Baum-Welch e HMM SOLVER com SPG. Teste realizado com o $M M O_{2,2}$. Todas as instâncias com 11 observações. . 74

5.18 Comparação entre BARON, HMM SOLVER com Baum-Welch e HMM SOLVER com SPG. Teste realizado em modelo de Markov oculto com mistura Bernoulli. Todas as instâncias testadas. . . . . . . . . . . . . . . . . . . . 75

5.19 Dispersão dos máximos globais para o $M M O_{2,2}$ com amostras de tamanho 10 . . . 78

5.20 Dispersão dos máximos globais para o $M M O_{2,2}$ com amostras de tamanho 11 . . . . 79

5.21 Dispersão dos máximos globais para o $M M O_{2,2}$ com amostras de tamanho 12. . . . 80

5.22 Dispersão dos máximos globais para o $M M O_{2,2}$ com amostras de tamanho 13. . . . 81

5.23 Dispersão dos máximos globais para o $M M O_{2,2}$ com amostras de tamanho 14. . . . 82

5.24 Dispersão dos máximos globais para o $M M O_{2,2}$ com amostras de tamanho 15. . . . 83 


\section{Lista de Tabelas}

2.1 Comparação das notações entre aritmética real ou de ponto flutuante e intervalar. . . 7

2.2 Tempo de execução de $N$ produtos em precisão dupla(coluna 2) e $N$ trocas no modo de arredondamento(coluna 3). Teste realizado com as funções declaradas no arquivo cfenv.h em $C++\ldots \ldots \ldots \ldots \ldots \ldots \ldots$

3.1 Comparação entre os algoritmos Gauss-Seidel intervalar e verifylss para limitar a solução de (3.4). A dimensão da matriz é $N, r$ é o raio da matriz $\boldsymbol{A} \ldots \ldots \ldots$

4.1 Comparação entre as normalizações padrão e nossa alternativa. . . . . . . . . . . . 28

5.1 Limitante superior obtido com os resolvedores BARON, HMM SOLVER com BaumWelch e HMM SOLVER com spg. Lista das 25 instâncias com maior soma da norma das diferenças dentre todas as 2025 testadas. . . . . . . . . . . . . . 76 


\section{Capítulo 1}

\section{Introdução}

Modelos de Markov ocultos (MMOs) são uma ferramenta importante em estatística e matemática aplicada. Eles têm sido utilizados nos últimos sessenta anos em áreas como reconhecimento de voz, biologia e finanças. De forma geral, um modelo de Markov oculto é composto por dois processos estocásticos. O primeiro é uma cadeia de Markov, que ao longo do trabalho será discreta no tempo e com número finito de estados. O segundo processo é um modelo de mistura, cuja densidade está condicionada ao estado da cadeia de Markov. O modelo é dito oculto pois não temos acesso ao primeiro processo diretamente. Isto é, observamos apenas as saídas de um processo. Trata-se portanto, de uma generalização das cadeias de Markov descritas em Norris (1998) e dos modelos de mistura, apresentados no Capítulo 1 de Zucchini e MacDonald (2009). A figura abaixo apresenta um exemplo de modelo de Markov oculto.

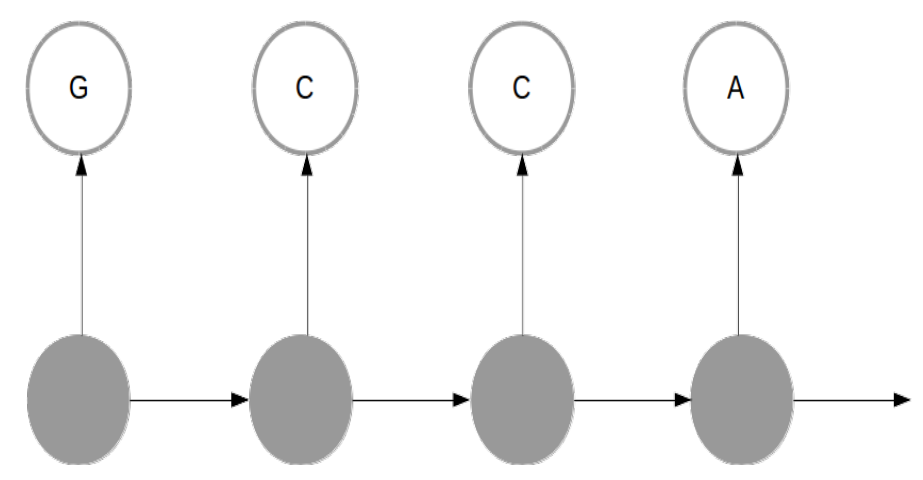

Figura 1.1: Representação de um modelo de Markov oculto

Os pontos em cinza representam os estados ocultos, associados à cadeia de Markov, enquanto os brancos são as saídas do modelo de mistura. A cada passo transitamos do estado $q_{n}$ para $q_{n+1}$ e realizamos uma observação. Um modelo que poderia ter gerado a figura é

$$
\pi^{\prime}=\left(\begin{array}{cc}
S_{1} & S_{2} \\
\hline \frac{1}{2} & \frac{3}{2}
\end{array}\right) \quad A=\left(\begin{array}{cc|cccc}
S_{1} & S_{2} \\
\hline \frac{1}{4} & \frac{3}{4} \\
\frac{1}{2} & \frac{1}{2}
\end{array}\right) \quad B=\left(\begin{array}{c|cccc}
A & A & C & G & T \\
\hline S_{1} & 0 & \frac{1}{3} & \frac{1}{3} & \frac{1}{3} \\
S_{2} & \frac{1}{2} & \frac{1}{4} & 0 & \frac{1}{4}
\end{array}\right) .
$$

O par $(A, \pi)$ define a cadeia de Marvok. O vetor $\pi$ é a distribuição inicial e $A$ a matriz de transição entre os estados. Cada linha de $B$ é uma distribuição no espaço $\{A, C, G, T\}$. A escolha de qual distribuição será usada a cada momento depende do estado oculto. Seja $o_{n}$ a $n$-ésima observação e $q_{n}$ o estado oculto correspondente. No exemplo acima temos:

- $q_{1}=S_{1}$, pois a probabilidade de observarmos $G$ a partir de $S_{2}$ é zero.

- O segundo simbolo foi emitido por $S_{1}$ com probabilidade 0.25 . 
- Para calcular $\mathbb{P}\left(o_{5}=T \mid q_{4}=S_{1}\right)$, utilizamos o teorema da probabilidade total

$$
\begin{aligned}
\mathbb{P}\left(o_{5}=T\right) & =\mathbb{P}\left(o_{5}=T, q_{5}=S_{1} \mid q_{4}=S_{1}\right)+\mathbb{P}\left(o_{5}=T, q_{5}=S_{2} \mid q_{4}=S_{1}\right) \\
& =\mathbb{P}\left(o_{5}, q_{5}=S_{1}\right) \mathbb{P}\left(q_{4}=S_{1}\right)+\mathbb{P}\left(o_{5}, q_{5}=S_{2}\right) \mathbb{P}\left(q_{4}=S_{1}\right) \approx 0.271
\end{aligned}
$$

Devido à simplicidade e flexibilidade dos MMOs, suas aplicações abrangem um grande espectro da matemática. Os primeiros trabalhos da área, de Baum e Petrie (1966) e Baum e Eagon (1967), discutem aplicações em ecologia. Atualmente, MMOs são usados em filogenética Pachter e Sturmfels (2007), genômica Burge e Karlin (1997) e Kulp et al. (1996), medicina Rani e Devi (2010) e epidemiologia Nunes et al. (2012).

Em sismologia, MMOs são interessantes pois os modelos de séries temporais consideram apenas ruídos Gaussianos. Dessa forma, eles não conseguem descrever adequadamente o comportamento de eventos sísmicos. Votsi et al. (2013) e Zucchini e MacDonald (2009) usam modelos ocultos com mistura de Poisson para descrever corretamente essas situações.

$\mathrm{Na}$ área de finanças, MMOs com mistura normal recebem o nome de switching Markov models. Destacamos o trabalho de Bhar e Hamori (2004), que se dedica à aplicação desses modelos em diversas situações econômicas.

Em engenharia, Rabiner (1990) e Levinson et al. (1983) aplicam modelos de Markov ocultos ao problema de reconhecimento de voz. Esta têm sido a aplicação mais importante desses modelos nos últimos anos.

Essas referências ilustram a capacidade dos MMOs em lidar com situações diversas. Não faremos uma revisão bibliográfica dos aspectos práticos e teóricos dos MMOs, porque a literatura da área é vasta. A busca pelo verbete hidden Markov model no zbmath, nos dá 1319 em dezembro de 2014. Desses, 258 foram publicados nos últimos 4 anos.

Ao longo da tese, estamos interessados nos aspectos computacionais dos modelos de Markov ocultos. Nesse sentido Rabiner (1990) e Levinson et al. (1983) destacam três problemas:

1. Dado um modelo de Markov oculto parametrizado por $\theta=(A, B, \pi)$, como no exemplo acima, e um conjunto de observações $O=o_{1}, \ldots, o_{T}$, calcular $\mathbb{P}(O \mid \theta)$,

2. Determinar a seqüência de estados ocultos $Q=q_{1}, \ldots, q_{T}$ que maximiza $\mathbb{P}(O, Q \mid \theta)$ e

3. Encontrar $\theta$ que maximiza $\mathbb{P}(\theta \mid O)$.

O problema 3 é o mais desafiador e há apenas algoritmos locais conhecidos para ele. Apesar dos artigos de Rabiner e Levinson serem da década de 80, esse problema ainda está aberto do ponto de vista da otimização global. Dentre as abordagens locais para resolvê-lo, destacamos Zucchini e MacDonald (2009), Cappé et al. (2005), Cappe e Moulines (2005), Bulla et al. (2010) e Barbu et al. (2012). Algoritmos genéticos tais como estudados em Aupetit et al. (2007) são uma solução parcial. Eles não garantem encontrar o máximo global de $\mathbb{P}(\theta \mid O)$.

A aritmética intervalar permite uma abordagem rigorosa do problema 3. Ela é adequada pois garante o cálculo rigoroso de funções. Por exemplo, suponha que desejamos somar dois valores, que não conhecemos exatamente, mas que pertencem aos intervalos [1.99, 2.01] e [2.99, 3.01]. Esta é uma situação comum quando operamos com aritmética de ponto flutuante. Nesses casos, arredondamos os valores segundo algum critério, e obtemos uma aproximação da soma. Em Moore (1979), o autor sugere que operemos com intervalos. De forma intuitiva temos

$$
[1.99,2.01]+[2.99,3.01]=[4.98,5.02] .
$$

Esse resultado dever ser interpretado da seguinte forma. Se somarmos um valor qualquer no intervalo $[1.99,2.01]$ a outro em $[2.99,3.01]$, o resultado estará contido em [4.98, 5.02].

Para entender porque a aritmética intervalar é adequada para otimização global, considere outro exemplo. Desejamos determinar todas as raízes de $f(x)=x^{3}+3 x^{2}+2$ no intervalo [-1,2]. O gráfico 1.2 foi construído utilizando as duas aritméticas, de ponto flutuante e intervalar. 


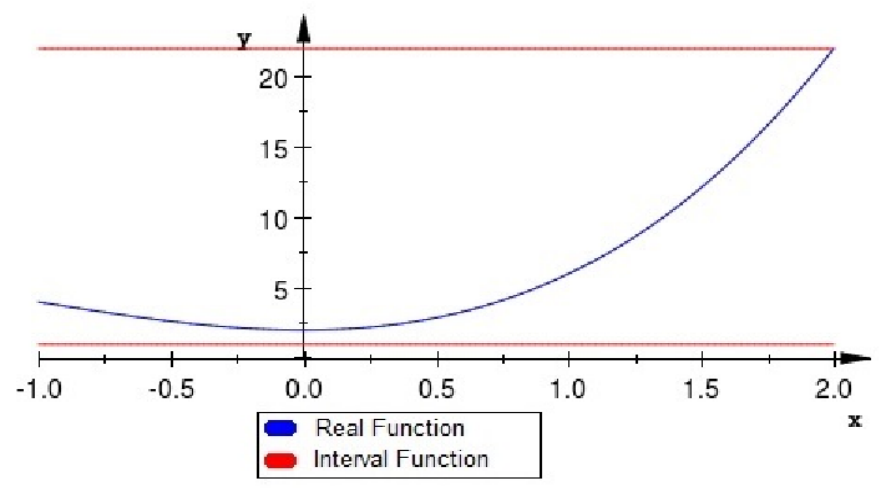

Figura 1.2: Gráfico de $x^{3}+3 x^{2}+2$ no intervalo $[-1,2]$.

Em aritmética de ponto flutuante ou real, a avaliação da função nos dá apenas informação local sobre $f$. Por exemplo, $f(1)=6$ nos diz que 1 não é raiz do problema, mas pouco além disso. Por outro lado, com aritmética intervalar obtemos como resultado o intervalo entre as linhas vermelhas. Algebricamente temos

$$
f(x)=x^{2} *(x+3)+2=[-1,2]^{2} *([-1,2]+3)+2=[2,22] .
$$

Concluímos então que não há raízes de $f$ em [-1,2]. Hansen e Walster (2004), Moore (1979), Kearfott (1996) e Neumaier (1990) exploram essa ideia na construção do interval branch and bound. Este é um dos algoritmos mais usados em otimização global.

Agora que introduzimos o problema e apresentamos uma ferramenta adequada para abordá-lo, enunciamos os objetivos da tese.

I Resolver o problema 3 do ponto de vista da otimização global.

II Resolver o problema 1 no caso intervalar. Descrever um algoritmo que, dado um modelo intervalar e um conjunto de observações, seja capaz de avaliar a probabilidade intervalar com eficiência e menor sobrestimação possível.

III Estudar mecanismos de aceleração da convergência do nosso algoritmo.

IV Comparar nossa implementação com o melhor resolvedor de problemas genéricos disponível.

V Realizar experimentos numéricos. Resolver problemas pequenos e estudar algumas propriedades de modelos de Markov ocultos com poucos parâmetros.

VI Estudar algoritmos locais do problema 3, tendo em vista sua aplicação no caso global.

Os objetivos I, III e IV são claros. Para justificar II, observamos que a qualidade do algoritmo global depende da avaliação de funções, com a menor sobrestimação possível. Por exemplo, na figura 2 não há sobrestimação do limitante superior, mas há no inferior. Se o excesso fizesse com que a linha vermelha fosse abaixo do zero, não poderíamos concluir a inexistência de raízes. Quanto mais próxima a imagem da função de sua estimativa intervalar, melhor será o algoritmo de otimização. Como o cálculo da função objetivo está intimamente ligado ao problema 1, vamos estuda-lo.

Com otimização global podemos realizar análises qualitativas em MMOs com poucos parâmetros. O objetivo $V$ é estudar a distribuição dos maximizadores globais. Construímos também as bacias de atração do algoritmo de Baum-Welch e gradiente projetado espectral, para alguns problemas selecionados. Com isso queremos estimar a probabilidade de métodos locais encontrarem o máximo global. 
Finalmente, destacamos que algoritmos locais são importantes em otimização global. A cada iteração eles nos dão limitantes inferiores para o máximo global. É importante analisar esses algoritmos em termos de eficiência e da qualidade das soluções.

A principal contribuição deste trabalho é a abordagem global para o problema 3. Nesse sentido, destacamos nossa modificação do interval branch and bound. Ela é capaz de determinar, em uma execução bem sucedida, todos os maximizadores globais de $\mathbb{P}(O \mid \theta)$. Além disso, ela é três vezes mais eficiente do que o resolvedor global mais usado atualmente, o BARON. Nosso algoritmo foi implementado em $C++11$ e está disponível em

$$
\text { www.ime.usp.br/ montanhe/hmm_solver.zip }
$$

Nosso trabalho não é o primeiro a usar aritmética intervalar em problemas estatísticos. Outras abordagens incluem o filtro de Kalman, descrito por Algahtani (2011) e Ahn et al. (2012), o algoritmo EM, dado por Wright e Kennedy (2000) e a solução global de regressões não lineares, proposta por Žilinskas e Žilinskas (2010). Por outro lado, até onde sabemos esta é a primeira contribuição que usa aritmética intervalar para estimar parâmetros em modelos de Markov ocultos.

O texto está dividido da seguinte forma. No capítulo 2, apresentamos a aritmética intervalar. O capítulo 3 introduz os modelos de Markov ocultos e sua principais propriedades. Apresentamos sua forma intervalar e discutimos o cálculo da distribuição estacionária.

No capítulo 4, estudamos o cálculo de $\mathbb{P}(O \mid \theta)$ e de suas derivadas nos casos real e intervalar. Mostramos como a programação linear leva a extensões intervalares estreitas e eficientes. Comparamos ainda nossa abordagem com a padrão.

O capítulo 5 é o mais importante da tese. Nele abordamos os tópicos a seguir.

- Derivamos condições necessárias para que $\theta^{*}$ seja um maximizador global.

- Analisamos os resolvedores locais, visando seu uso no algoritmo global.

- Descrevemos interval branch and bound e os procedimentos de aceleração da convergência.

- Comparamos os resultados de nossa implementação com o BARON Sahinidis (2014), que é resolvedor global mais usado atualmente. 


\section{Capítulo 2}

\section{Aritmética Intervalar}

Neste capítulo apresentamos a aritmética intervalar. Começamos com alguns comentários históricos, que ajudam a entender suas motivações e alcance. Os primeiros trabalhos na área são de Sunaga (1958) e Moore (1962). Eles têm em comum a necessidade de estimar erros cometidos em longas seqüências de cálculos.

Em meados do século passado, a construção dos primeiros computadores levou a uma revolução na computação científica. Problemas que antes não podiam ser resolvidos, passaram a ser tratados em tempo razoável. Essa revolução trouxe consigo a necessidade de avaliar, da forma mais simples possível, erros de representação e arredondamento nos cálculos. A aritmética intervalar é uma resposta a essa necessidade. Por isso, as primeiras publicações na área foram voltadas à determinação automática de erros.

$\mathrm{Na}$ década de 60, a aritmética intervalar encontrou aplicações além de seu escopo inicial. Moore (1966) apresenta condições suficientes para existência e unicidade de raízes em sistemas não lineares. Essas condições são mais fáceis de testar do que aquelas do teorema de Kantorovich. Para uma comparação entre os dois métodos, veja Rall (1980).

Atualmente, vários resultados em matemática aplicada são obtidos através de análise intervalar. Os casos mais famosos são a demonstração da conjectura de Kepler, feita por Hales et al. (2009) e a prova da existência de atratores de Lorenz, descrita em Tucker (2002). Mascarenhas (1997) e Mascarenhas (2014) usam aritmética intervalar na demonstração de resultados em otimização.

Outra aplicação importante é a construção de algoritmos para otimização global. Esse problema é equivalente a determinar todas as raízes de um sistema não linear. O primeiro algoritmo nesse sentido é chamado de Moore-Skelboe, ou interval branch and bound e é descrito em Moore (1977). Ele implementa uma árvore de busca, onde cada nó é uma pequena parte do domínio. A aritmética intervalar garante que nós contendo máximos globais, não serão descartados.

Embora o algoritmo de Moore-Skelboe seja rigoroso, a busca na árvore pode ser bastante custosa. Vários autores estudaram formas de melhorar seu desempenho. Dentre eles, destacamos Hansen e Sengupta (1981), Pedamallu et al. (2008), Martinez et al. (2004) e Csendes (2004). Neste trabalho especializamos o interval branch and bound para estimar parâmetros em modelos de Markov ocultos.

Este capítulo estabelece a notação que usamos, além de mostrar os principais resultados da aritmética. Nossa abordagem segue de perto os trabalhos de Kearfott (1996) e Hansen e Walster (2004). A notação segue o padrão estabelecido em Kearfott et al. (2010).

Na seção 2.1 apresentamos os resultados fundamentais da área. Em 2.2 discutimos a implementação da aritmética intervalar. A seção 2.3 dedica-se à solução de sistemas lineares intervalares, a partir de uma adaptação do algoritmo de Gauss-Seidel. Concluímos o capítulo com um exemplo que mostra como o interval branch and bound funciona. 


\subsection{Conceitos fundamentais}

Começamos com um exemplo que ilustra a necessidade da aritmética intervalar. Considere um computador que implementa o padrão IEEE754, descrito em 754 (1985). Assuma que $\mathbb{M}(F)$ é o conjunto de números representáveis na máquina, em função do tipo de ponto flutuante. Geralmente $F$ é float ou double.

Sabemos que $\frac{1}{3} \notin \mathbb{M}(F)$, independente de $F$ ser float ou double. Ao realizar cálculos com essa quantidade, consideramos uma aproximação $\frac{1}{3} \approx \tilde{x} \in \mathbb{M}(f)$. Conforme os cálculos evoluem, acumulamos erros que podem ou não ser significativos.

A aritmética intervalar contempla automaticamente erros de representação e arredondamento. Ao invés de usarmos aproximações de valores reais, construímos intervalos que os contém. Por exemplo, $[0.25,0.5]$ tem seus extremos em $\mathbb{M}(F)$ e contém $\frac{1}{3}$. Suponha que desejamos calcular $\frac{1}{3} * b$, onde $b \in[0.75,1]$. Para avaliar automaticamente o erro da operação, procuramos um intervalo $X$ que satisfaça

- $\frac{b}{3} \in X$.

- $X$ depende apenas dos intervalos $[0.25,0.5]$ e $[0.75,1]$, não de $\frac{1}{3}$ e $b$.

- Os extremos de $X$ pertencem à $\mathbb{M}(F)$.

Se $X$ satisfaz as três condições, tomamos seu ponto médio como aproximação do produto. $\mathrm{O}$ raio do intervalo será um limitante rigoroso do erro. Na prática, a construção do intervalo requer um procedimento em dois passos

1. Definir operações que garantam as duas primeiras exigências.

2. Arredondar corretamente os extremos para garantir o último requisito.

Mostramos como calcular $X$ para as quatro operações elementares. Nesta seção lidamos apenas com intervalos reais. Na próxima seção, discutimos a implementação dessas operações.

\subsubsection{Operações Fundamentais}

A aritmética intervalar clássica considera apenas intervalos fechados. Eles são denotados por $\boldsymbol{x}=[\underline{x}, \bar{x}]$, onde $-\infty<\underline{x} \leq \bar{x}<\infty$. O conjunto dos intervalos reais é dado por $\mathbb{I R}$. Uma caixa é um vetor de intervalos. Em geral não distinguimos escalares e caixas, grafando ambos por $\boldsymbol{x}$. Quando houver possibilidade de dúvida, denotamos as caixas por $\overrightarrow{\boldsymbol{x}}$.

Funções intervalares são aquelas cujo domínio e contra-domínio são $\mathbb{R}$ ou $\mathbb{R}^{n}$. Elas também são representadas em negrito, $\boldsymbol{f}: \mathbb{R}^{n} \rightarrow \mathbb{I}$ ou $\boldsymbol{F}: \mathbb{R}^{n} \rightarrow \mathbb{R}^{m}$. Há funções importantes que levam $\mathbb{I} \mathbb{R}$ em $\mathbb{R}$. O mínimo e o máximo de um intervalo $\boldsymbol{x}$ são dados por $\inf (\boldsymbol{x})$ e $\sup (\boldsymbol{x})$. Além delas, temos o ponto médio, raio, comprimento e a norma de um intervalo, dados respectivamente por

$$
\begin{aligned}
\operatorname{mid}(\boldsymbol{x}) & =\frac{\inf (\boldsymbol{x})+\sup (\boldsymbol{x})}{2} \\
\operatorname{rad}(\boldsymbol{x}) & =\frac{\sup (\boldsymbol{x})-\inf (\boldsymbol{x})}{2} \\
w(\boldsymbol{x}) & =\sup (\boldsymbol{x})-\inf (\boldsymbol{x}) \\
\|\boldsymbol{x}\| & =\max (|\inf (\boldsymbol{x})|,|\sup (\boldsymbol{x})|) .
\end{aligned}
$$

A generalização de inf, sup mid e rad para caixas ou matrizes intervalares é imediata. Se $\overrightarrow{\boldsymbol{v}}$ e $\boldsymbol{M}$ são um vetor e uma matriz intervalares então

$$
\begin{aligned}
w(\overrightarrow{\boldsymbol{v}}) & =\max \left(w\left(\boldsymbol{v}_{\mathbf{1}}\right), \ldots, w\left(\boldsymbol{v}_{\boldsymbol{n}}\right)\right) \\
\|\overrightarrow{\boldsymbol{v}}\| & =\max \left(\left\|\boldsymbol{v}_{\mathbf{1}}\right\|, \ldots,\left\|\boldsymbol{v}_{\boldsymbol{n}}\right\|\right) \\
\|M\| & =\max _{i} \sum_{j}\left\|\boldsymbol{m}_{\boldsymbol{i j}}\right\|
\end{aligned}
$$


A tabela abaixo resume a notação adotada, comparando as aritméticas real e intervalar.

\begin{tabular}{|c|c|c|}
\hline Escalar & Real & Intervalar \\
Vetor & $x$ ou $\vec{x}$ & $\boldsymbol{x}$ ou $\overrightarrow{\boldsymbol{x}}$ \\
Matriz & $M$ & $\boldsymbol{M}$ \\
Função & $f$ ou $F$ & $\boldsymbol{f}$ ou $\boldsymbol{F}$ \\
\hline
\end{tabular}

Tabela 2.1: Comparação das notações entre aritmética real ou de ponto flutuante e intervalar.

As operações fundamentais entre intervalos são definidas a seguir. Sejam $\boldsymbol{x}=[\underline{x}, \bar{x}]$ e $\boldsymbol{y}=[\underline{y}, \bar{y}]$, então

$$
\boldsymbol{x} \otimes \boldsymbol{y}=\{x \otimes y \mid x \in \boldsymbol{x}, y \in \boldsymbol{y}\} \text { para } \otimes \in\{+,-, *, /\} .
$$

Algebricamente temos

$$
\begin{aligned}
\boldsymbol{x}+\boldsymbol{y}= & {[\inf (x)+\inf (y), \sup (x)+\sup (y)], } \\
\boldsymbol{x}-\boldsymbol{y}= & {[\inf (x)-\sup (y), \sup (x)-\inf (y)], } \\
\boldsymbol{x} * \boldsymbol{y}= & {[\min \{\inf (x) \inf (y), \inf (x) \sup (y), \sup (x) \inf (y), \sup (x) \sup (y)\}, \sup (x) \sup (y)\}], \sup (x) \inf (y), \sup (x), \quad 0 \notin \boldsymbol{y}, } \\
& \max \{\inf (x) \inf (y), \inf (x) \sup (y) \\
\frac{1}{\boldsymbol{y}}= & {\left[\frac{1}{\sup (y)}, \frac{1}{\inf (y)}\right] \quad 0 \quad 0 \notin \boldsymbol{y} . } \\
\frac{\boldsymbol{x}}{\boldsymbol{y}}= & \boldsymbol{x} * \frac{1}{\boldsymbol{y}} \quad 0 .
\end{aligned}
$$

Todo ponto $x \in \mathbb{R}$ está identificado com o intervalo degenerado $\boldsymbol{x}=[x, x]$. Dessa forma, as quatro operações fundamentais ficam bem definidas entre intervalos e valores reais.

Proposição 1 Sejam $\boldsymbol{a}, \boldsymbol{b}$ e $\boldsymbol{c}$ intervalos em $\mathbb{I R}$, então

$$
\begin{aligned}
\boldsymbol{a}+(\boldsymbol{b}+\boldsymbol{c}) & =(\boldsymbol{a}+\boldsymbol{b})+\boldsymbol{c}, \\
\boldsymbol{a}+\boldsymbol{b} & =\boldsymbol{b}+\boldsymbol{a}, \\
\boldsymbol{a}(\boldsymbol{b c}) & =(\boldsymbol{a b}) \boldsymbol{c}, \\
\boldsymbol{a b} & =\boldsymbol{b} \boldsymbol{a}, \\
\boldsymbol{a}+\boldsymbol{b} & =\boldsymbol{b}+\boldsymbol{c} \Rightarrow \boldsymbol{a}=\boldsymbol{c}, \\
\boldsymbol{a}(\boldsymbol{b}+\boldsymbol{c}) & \subseteq \boldsymbol{a b}+\boldsymbol{a c} .
\end{aligned}
$$

Além disso, $\mathbf{0}=[0,0]$ e $\mathbf{1}=[1,1]$ são os únicos intervalos que satisfazem

$$
\begin{aligned}
\mathbf{0}+\boldsymbol{a} & =\boldsymbol{a}, \\
\mathbf{0} \boldsymbol{a} & =0, \\
\mathbf{1} \boldsymbol{a} & =\boldsymbol{a} \quad \forall \boldsymbol{a} \in \mathbb{R} \mathbb{R} .
\end{aligned}
$$

A proposição segue imediatamente da definição de intervalo e das operações (2.2) - (2.6). A maior diferença entre as aritméticas real e intervalar é a propriedade distributiva. Para intervalos, vale apenas a sub distributividade Segue daí que para dois intervalos $\boldsymbol{a}$ e $\boldsymbol{b}, \boldsymbol{a}(\boldsymbol{b}-\boldsymbol{b}) \neq \mathbf{0}$ a menos que um deles seja degenerado. Além disso $\boldsymbol{a}-\boldsymbol{a}=[\underline{a}-\bar{a}, \bar{a}-\underline{a}]$ e não $\mathbf{0}$, a menos que $\boldsymbol{a}$ seja degenerado.

\subsubsection{Propriedade de Inclusão}

A principal finalidade da aritmética intervalar é obter limitantes inferiores e superiores do contradomínio de funções reais. Nesse sentido, a propriedade de inclusão, a variação de uma função e 
a extensão intervalar são conceitos importantes.

Definição 1 Seja $\boldsymbol{f}: \mathbb{R} \rightarrow \mathbb{R}$ uma função intervalar. Diremos que $\boldsymbol{f}$ tem propriedade de inclusão se

$$
\boldsymbol{y} \subseteq \boldsymbol{x} \Rightarrow \boldsymbol{f}(\boldsymbol{y}) \subseteq \boldsymbol{f}(\boldsymbol{x}) .
$$

Na prática, a maioria das funções intervalares tem propriedade de inclusão. Um contra-exemplo é dado em Moore et al. (2009). Se $\boldsymbol{f}(\boldsymbol{x})=\operatorname{mid}(\boldsymbol{x})+\frac{1}{2} \boldsymbol{x}$ então $\boldsymbol{y}=[2,3] \subseteq \boldsymbol{x}=[1,3]$ e

$$
f(\boldsymbol{y})=[2.5,3.5] \nsubseteq[3.5,4]=f(\boldsymbol{x}) .
$$

Definição 2 Seja $f: \mathbb{R}^{n} \rightarrow \mathbb{R}$ contínua. A função de conjuntos $\overline{\boldsymbol{f}}: \mathbb{R}^{n} \rightarrow \mathbb{I}$ dada por

$$
\overline{\boldsymbol{f}}(\boldsymbol{x}):=\{y \in \mathbb{R} \text { tal que existe } x \in \boldsymbol{x} \text { com } y=f(x)\}
$$

é chamada variação de $f$.

Definição 3 A função $f: \mathbb{R}^{n} \rightarrow \mathbb{I} \mathbb{R}$ é extensão intervalar de $f$ se para quaisquer $x_{1}, \ldots, x_{n} \in \mathbb{R}^{n}$

$$
\boldsymbol{f}\left(x_{1}, \ldots, x_{n}\right)=f\left(x_{1}, \ldots, x_{n}\right) .
$$

Para qualquer $x \in \mathbb{R}^{n}$, a avaliação de $\boldsymbol{f}$ na caixa degenerada $\boldsymbol{x}$ resulta no intervalo degenerado $[f(x), f(x)]$. Com essas definições, podemos enunciar o teorema fundamental da aritmética intervalar, demonstrado em Moore et al. (2009).

Teorema 1 (Teorema Fundamental da aritmética intervalar - TFAI) Se $\boldsymbol{f}$ tem propriedade de inclusão e é extensão intervalar de $f$ então $\overline{\boldsymbol{f}}(\boldsymbol{x}) \subseteq \boldsymbol{f}(\boldsymbol{x})$.

Ao avaliarmos (2.2) - (2.6) em intervalos degenerados $\boldsymbol{x}=[x, x]$ e $\boldsymbol{y}=[y, y]$, o resultado coincidirá com a avaliação real. Além disso, por (2.1), as operações elementares tem propriedade de inclusão. Dessa forma, o TFAI vale para as operações fundamentais. A próxima proposição garante que o resultado continua válido para composição de funções.

Proposição 2 Se $\boldsymbol{f}: \mathbb{R}^{m} \rightarrow \mathbb{I}$ e $\boldsymbol{g}: \mathbb{I}^{n} \rightarrow \mathbb{I}^{m}$ são funções intervalares nas condições do TFAI, então $\overline{\boldsymbol{f} \circ \boldsymbol{g}}(\boldsymbol{x}) \subseteq \boldsymbol{f} \circ \boldsymbol{g}(\boldsymbol{x})$.

Demonstração - Por hipótese, $\overline{\boldsymbol{g}}(\boldsymbol{x}) \subseteq \boldsymbol{g}(\boldsymbol{x})$. Assim, ao avaliarmos $\boldsymbol{f} \circ \boldsymbol{g}$ estamos avaliando todos os pontos da variação de $g$. Como $\boldsymbol{f}$ também satisfaz as condições do teorema, segue o resultado. $\square$

Definição 4 Seja $f: \mathbb{R}^{n} \rightarrow \mathbb{R}^{m}$ composta por operações elementares e funções padrão. A função intervalar $\boldsymbol{f}: \mathbb{R}^{n} \rightarrow \mathbb{I}^{m}$ obtida substituindo-se as operaçôes reais por intervalares, é dita extensão natural de $f$.

\subsubsection{Teorema do Valor Médio Intervalar}

O principal resultado da seção é teorema do valor médio intervalar. Ele generaliza para caixas a proposição que no caso real vale apenas para funções unidimensionais.

Teorema 2 (Teorema do Valor Médio Intervalar) Sejam $\boldsymbol{x} \in \mathbb{R}^{n}$, x e y pontos em $\boldsymbol{x}$ e $f$ : $\mathbb{R}^{n} \rightarrow \mathbb{R}^{n}$ diferenciável. Se $\boldsymbol{f}$ e $\boldsymbol{J}$ são extensões intervalares de $f$ e de sua matriz jacobiana, então

$$
f(y) \in \boldsymbol{f}\left(\boldsymbol{x}^{I}\right)+\boldsymbol{J}(\boldsymbol{x})(y-x)
$$

onde $\boldsymbol{x}^{I}$ é a caixa degenerada $\boldsymbol{x}^{I}=[x, x]$. 
Demonstração - Seja $i=1, \ldots n$ e considere a função $h_{i}(t)=f_{i}(x+t(y-x))$. Segue do teorema do valor médio real que

$$
h_{i}(1)=h_{i}(0)+h_{i}^{\prime}\left(\varepsilon_{i}\right)(1-0), \text { para algum } \varepsilon_{i} \in[0,1] .
$$

Se chamamos de $J_{i}$ o vetor gradiente de $f_{i}$ então a igualdade acima nos dá

$$
h_{i}(1)=h_{i}(0)+J_{i}\left(x+\varepsilon_{i}(y-x)\right)(y-x), \text { para algum } \varepsilon_{i} \in[0,1] .
$$

Note que $h_{i}(1)=f_{i}(y)$ e $h_{i}(0)=f_{i}(x)$. Como $x$ e $y$ pertencem a $\boldsymbol{x}$ e esse é um conjunto convexo, temos que $x+\varepsilon_{i}(y-x) \in \boldsymbol{x}$. Além disso, como $\boldsymbol{f}$ e $\boldsymbol{J}$ são extensões intervalares de $f$ e $J$ temos que

$$
f_{i}(y) \in \boldsymbol{f}_{i}(x)+\boldsymbol{J}_{i}(\boldsymbol{x})(y-x) .
$$

Agrupando as expressões em forma matricial segue o resultado.

\section{$2.2 \quad$ Aspectos Computacionais}

Na seção anterior apresentamos a aritmética intervalar com extremos em $\mathbb{R}$. Agora, discutimos os detalhes de implementação dessa aritmética.

\subsubsection{Modo de Arredondamento e operações elementares}

Considere o conjunto de números de ponto flutuante $\mathbb{M}(F)$. Na prática, a aritmética intervalar tem os extremos dos intervalos em $\mathbb{M}(F)$, não em $\mathbb{R}$. Precisamos então definir regras para que a propriedade de inclusão se preserve a cada operação. Nesse ponto, o modo de arredondamento desempenha papel central. Ele determina como será a aproximação de valores não representáveis por números de ponto flutuante.

Suponha que o resultado de uma operação é $r \notin \mathbb{M}(F)$, conforme ilustrado na figura abaixo.

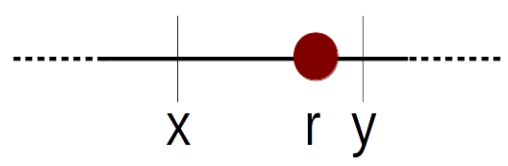

Figura 2.1: Representação dos diferentes modos de arredondamento.

Os pontos $x, y \in \mathbb{M}(F)$ são os mais próximos de $r$. A política de arredondamento diz para qual valor $r$ será aproximado. As três formas mais comuns de arredondamento são

- para o valor mais próximo.

- para cima, que denotamos por $\Delta($.$) .$

- para baixo, onde escrevemos $\nabla($.$) .$

Se o modo de arredondamento está para o mais próximo, ou para cima então $r$ será aproximado por $y$. Se estamos preocupados em determinar um intervalo que contenha $r$, então devemos realizar a operação com o modo de arredondamento para baixo.

Mudar o modo de arredondamento a cada operação é a principal exigência das implementações de aritmética intervalar. Em geral essa não é uma restrição grave, pois o IEEE754 permite realizar as quatro operações especificando a direção de arredondamento. Por outro lado, essa é uma operação custosa que deve ser evitada. Veja a tabela abaixo. 


\begin{tabular}{|c|c|c|c|}
\hline $\mathrm{N}$ & double(s) & Round(s) & Round / double \\
\hline $1.0 \mathrm{e} 4$ & 0.0189 & 0.2064 & 10.9319 \\
\hline $5.0 \mathrm{e} 4$ & 0.0802 & 1.0015 & 12.4998 \\
\hline $1.0 \mathrm{e} 5$ & 0.1500 & 1.9861 & 13.2484 \\
\hline $5.0 \mathrm{e} 5$ & 0.7664 & 10.1770 & 13.2795 \\
\hline $1.0 \mathrm{e} 6$ & 1.4947 & 20.0194 & 13.3942 \\
\hline $5.0 \mathrm{e} 6$ & 7.4582 & 100.5616 & 13.4835 \\
\hline $1.0 \mathrm{e} 7$ & 15.1403 & 201.7591 & 13.3260 \\
\hline
\end{tabular}

Tabela 2.2: Tempo de execução de $N$ produtos em precisão dupla(coluna 2) e $N$ trocas no modo de arredondamento(coluna 3). Teste realizado com as funções declaradas no arquivo cfenv.h em $C++$.

A tabela nos diz que mudar o modo de arredondamento é, em média, 13 vezes mais custoso que o produto em precisão dupla. $\mathrm{O}$ experimento foi realizado em $\mathrm{C}++11$, com o compilador icc ++14.0 .2$ e opções de compilação -O3, -frounding-math e -NDEBUG.

A cada iteração realizamos $N$ multiplicações com valores aleatórios do tipo double, escolhidos no intervalo $[0,1]$. Comparamos o resultado com $N$ trocas no modo de arredondamento. Para evitar otimizações indevidas do compilador, a cada troca no modo de arredondamento realizamos a soma de dois valores aleatórios. O tempo das somas é subtraído do resultado.

Agora podemos definir as operações intervalares básicas em termos computacionais. A partir de $(2.2)$ - (2.6) e das funções $\nabla()$ e $\Delta()$ discutidas acima temos

$$
\begin{aligned}
\boldsymbol{x}+\boldsymbol{y}= & {[\nabla(\inf (x)+\inf (y)), \Delta(\sup (x)+\sup (y))] } \\
\boldsymbol{x}-\boldsymbol{y}= & {[\nabla(\inf (x)-\sup (y)), \Delta(\sup (x)-\inf (y))] } \\
\boldsymbol{x} * \boldsymbol{y}= & {[\nabla(\min \{\inf (x) \inf (y), \inf (x) \sup (y), \sup (x) \inf (y), \sup (x) \sup (y)\}),} \\
& \Delta(\max \{\inf (x) \inf (y), \inf (x) \sup (y), \sup (x) \inf (y), \sup (x) \sup (y)\})] \\
\frac{1}{\boldsymbol{y}}= & {\left[\nabla\left(\frac{1}{\sup (y)}\right), \Delta\left(\frac{1}{\inf (y)}\right)\right] \quad 0 \notin \boldsymbol{y} } \\
\frac{\boldsymbol{x}}{\boldsymbol{y}}= & \boldsymbol{x} * \frac{1}{\boldsymbol{y}} \quad 0 \notin \boldsymbol{y} .
\end{aligned}
$$

A implementação dessas operações garante o cálculo intervalar rigoroso. Por outro lado, esse rigor exige um número significativo de trocas no modo de arredondamento.

A extensão intervalar de funções monótonas requer a avaliação dos extremos do intervalo. Geralmente isso é feito usando a MPFR, descrita em Fousse et al. (2007). Essa operação tem custo maior ainda, pois é feita em software. A extensão de funções não monótonas é mais complicada e foge ao escopo da tese. O leitor interessado pode consultar Rump (2001).

\subsubsection{Divisão estendida}

A divisão tradicional exige que o denominador $\boldsymbol{y}$ não contenha zero. No entanto, há situações em que é útil considerar que $0 \in \boldsymbol{y}$. É o caso do método de Gauss-Seidel intervalar, discutido adiante. Apresentamos a divisão estendida proposta por Ratz (1996). Ela geralmente é usada em otimização global, veja por exemplo o GLOBSOL Kearfott (1996).

A divisão estendida exige a generalização do conceito de intervalo para lidar com $\pm \infty$. Como nossa preocupação é mais computacional do que teórica, nos restringimos aos problemas de implementação que a nova divisão demanda. Vamos supor que os intervalos tem seus extremos em $\mathbb{M}(F)$, em um computador que implementa o IEEE754. Nesse padrão, os símbolos $-\infty, \infty$ e $N a N$ fazem parte de $\mathbb{M}(F)$.

Definição 5 Sejam $\boldsymbol{a}=\left[a_{1}, a_{2}\right]$ e $\boldsymbol{b}=\left[b_{1}, b_{2}\right]$ com extremos em $\mathbb{M}(F)-\{-\infty, \infty, N a N\}$, então 


$$
\frac{\boldsymbol{a}}{\boldsymbol{b}}= \begin{cases}{\left[\nabla\left(\frac{a_{2}}{b_{1}}\right), \infty\right]} & \text { se } a_{2}<0 \text { e } b_{1}<b_{2}=0 \\ {\left[-\infty, \Delta\left(\frac{a_{2}}{b_{2}}\right)\right] \cup\left[\nabla\left(\frac{a_{2}}{b_{1}}\right), \infty\right]} & \text { se } a_{2}<0 \text { e } b_{1}<0<b_{2} \\ {\left[-\infty, \Delta\left(\frac{a_{2}}{b_{2}}\right)\right]} & \text { se } a_{2}<0 \text { e } 0=b_{1}<b_{2} \\ {[-\infty, \infty]} & \text { se } a_{1}<0<a_{2} \text { e } b_{1}<0<b_{2} \\ {\left[-\infty, \Delta\left(\frac{a_{1}}{b_{1}}\right)\right]} & \text { se } a_{1}>0 \text { e } b_{1}<b_{2}=0 \\ {\left[-\infty, \Delta\left(\frac{a_{1}}{b_{1}}\right)\right] \cup\left[\nabla\left(\frac{a_{1}}{b_{2}}\right), \infty\right]} & \text { se } a_{1}>0 \text { e } b_{1}<0<b_{2} \\ {\left[\nabla\left(\frac{a_{1}}{b_{2}}\right), \infty\right]} & \text { se } a_{1}>0 \text { e } 0=b_{1}<b_{2} \\ \mathrm{NaN} & \text { se } 0 \notin \boldsymbol{a} \text { e } \boldsymbol{b}=[0,0]\end{cases}
$$

O resultado da divisão intervalar estendida pode ser um ou dois intervalos disjuntos. Essa divisão mantém o rigor da aritmética intervalar. Por exemplo, se $\boldsymbol{a}=[2,3]$ e $\boldsymbol{b}=[-1,2]$, então o resultado da divisão são os intervalos $\boldsymbol{r}_{1}=[-\infty,-2]$ e $\boldsymbol{r}_{2}=[1, \infty]$. Não há $p \in(-2,1)$ tal que $\frac{a}{b}=p$ para $a \in \boldsymbol{a}$ e $b \in \boldsymbol{b}$.

\subsection{Sistemas Lineares Intervalares}

Considere o sistema linear $\boldsymbol{A} \boldsymbol{x}=\boldsymbol{b}$, onde $\boldsymbol{A} \in \mathbb{I}^{n x n}$ e $\boldsymbol{b}, \boldsymbol{x} \in \mathbb{I}^{n}$. A solução desse problema é o conjunto

$$
\mathcal{S}:=\left\{x \in \mathbb{R}^{n} \text { tal que existem } A \in \boldsymbol{A} \text { e } b \in \boldsymbol{b} \operatorname{com} A x=b\right\} .
$$

Diremos que uma matriz intervalar $\boldsymbol{A}$ é regular se não contém matrizes singulares. Ao contrário do caso real, determinar $\mathcal{S}$ é um problema $N P$-Completo, mesmo para matrizes regulares. Considere o sistema abaixo, extraído de Kearfott (1996).

$$
\left(\begin{array}{cc}
{[2,4]} & {[-2,1]} \\
{[-1,2]} & {[2,4]}
\end{array}\right) \boldsymbol{x}=\left(\begin{array}{c}
{[-2,2]} \\
{[-2,2]}
\end{array}\right)
$$

Apesar da matriz $\boldsymbol{A}$ ser regular, o conjunto $\mathcal{S}$ é complicado. Veja a Figura 2.2.

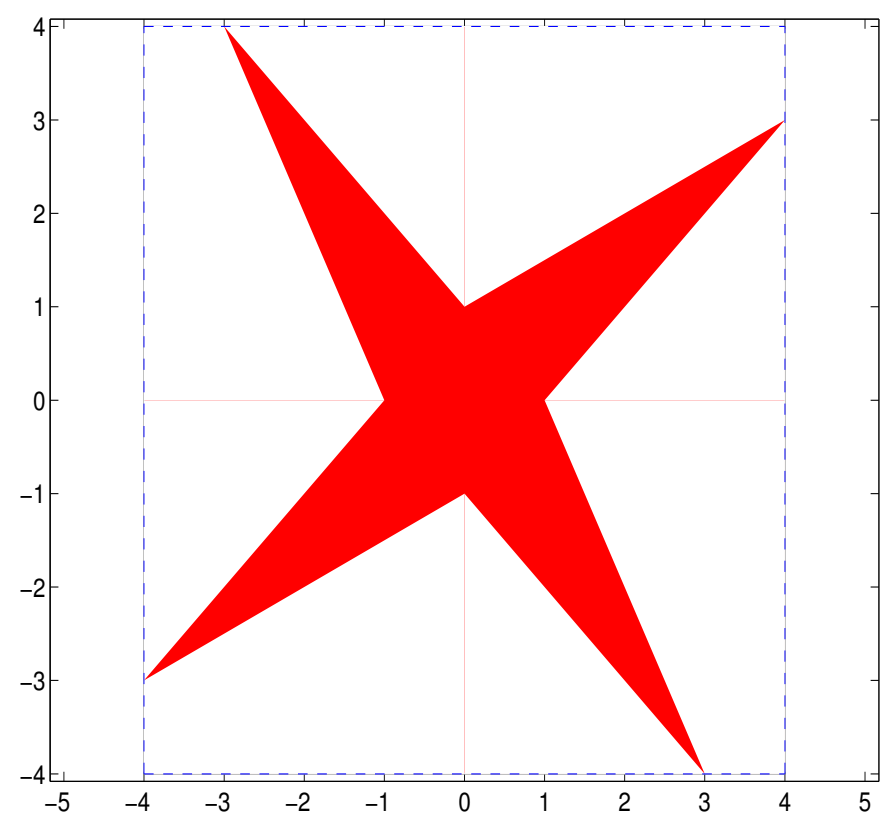

Figura 2.2: Solução de (2.15) obtida com a função plotlinsol do INTLAB. Em vermelho o conjunto $\mathcal{S} e$ tracejado em azul seu casco intervalar. 
Definição 6 (Casco Intervalar) O casco intervalar $\boldsymbol{h}$ de um conjunto $\mathcal{S}$ é a intersecção de todas as caixas que o contém.

Determinar $\boldsymbol{h}$ também é um problema NP-Completo, segundo Hansen e Walster (2004). Apresentamos o método de Gauss-Seidel intervalar. Ele determina uma caixa $\boldsymbol{x} \supseteq \boldsymbol{h} \supseteq \mathcal{S}$. Outras alternativas para esse problema, são a eliminação Gaussiana intervalar e o operador de Krawczyk, descritos em Kearfott (1996).

\subsubsection{Gauss-Seidel Intervalar}

Considere o sistema linear intervalar

$$
\boldsymbol{A}(\boldsymbol{y}-\operatorname{mid}(\boldsymbol{x}))=\boldsymbol{b}
$$

Aqui, $\boldsymbol{x}$ é a caixa inicial onde procuramos a aproximação do casco intervalar de $\mathcal{S}$. Esse é o sistema que aparece no método de Newton intervalar e por isso vamos estudar essa forma modificada. $\mathrm{O}$ método de Gauss-Seidel intervalar é dado por

$$
\boldsymbol{x}_{i}^{\prime}=\boldsymbol{x}_{i} \cap \boldsymbol{y}_{i}, \quad i=1 \ldots n,
$$

onde

$$
\boldsymbol{y}_{i}=\operatorname{mid}\left(\boldsymbol{x}_{i}\right)+\frac{\boldsymbol{r}_{i}}{\boldsymbol{a}_{i i}}
$$

$\mathrm{e}$

$$
\boldsymbol{r}_{i}=\boldsymbol{b}_{\boldsymbol{i}}-\sum_{\substack{j=1 \\ j \neq i}}^{n} \boldsymbol{a}_{i j}\left(\boldsymbol{x}_{j}-\operatorname{mid}\left(\boldsymbol{x}_{j}\right)\right) .
$$

O intervalo $\boldsymbol{a}_{i i}$ pode conter zero. Por isso, dividimos o algoritmo em dois passos. O primeiro passo é o de Gauss-Seidel não estendido. Nele aplicamos (2.17)- (2.19) às linhas $i$ onde $0 \notin \boldsymbol{a}_{i i}$.

Nesse estágio garantimos que $\boldsymbol{y}_{i}$ é um intervalo, pois não há divisão por intervalos que contenham zero. Segue daí que $\boldsymbol{x}_{i}^{\prime}$ pode ser um intervalo ou o conjunto vazio. No primeiro caso temos uma nova estimativa para $\boldsymbol{h}$ na direção $i$. Essa nova estimativa é no mínimo tão boa quanto a anterior. Caso $\boldsymbol{x}_{i}^{\prime}$ seja vazio, o TFAI garante que $\boldsymbol{x}$ não contém soluções do sistema.

O segundo passo é o de Gauss-Seidel estendido. Ele consiste em aplicar (2.17) - (2.19) às linhas $i$ onde $0 \in \boldsymbol{a}_{i i}$. Para tanto recorremos à divisão estendida (2.14). O resultado da operação pode ser o conjunto vazio, um intervalo ou dois.

Nos dois primeiros casos caímos nas mesmas situações do parágrafo anterior. Caso a divisão estendida resulte em duas caixas na coordenada $i$, guardamos essa informação e o diâmetro do intervalo que separa os resultados. Ao contrário do primeiro estágio, neste iteramos apenas uma vez sobre o conjunto de índices. Ao final, procuramos entre os índices com dois intervalos, aquele com maior diâmetro. Se $k$ é esse índice, então chamamos de $\boldsymbol{x}_{k}^{\prime}$ e $\boldsymbol{x}_{k}^{\prime \prime}$ os intervalos resultantes do segundo passo de Gauss-Seidel. Concluímos o algoritmo devolvendo duas caixas $\boldsymbol{x}^{1}$ e $\boldsymbol{x}^{2}$. Essas caixas são tais que $\boldsymbol{x}_{k}^{1}=\boldsymbol{x}_{k}^{\prime}, \boldsymbol{x}_{k}^{2}=\boldsymbol{x}_{k}^{\prime \prime}$ e $\boldsymbol{x}_{i}^{1}=\boldsymbol{x}_{i}^{2}$ para $i \neq k$.

Apresentamos no apêndice A o pseudo-código dos dois passos do método de Gauss-Seidel intervalar.

\subsubsection{Pré-Condicionamento}

Seja $M$ uma matriz quadrada de tamanho $n$. O raio espectral de $M$ é dado por $\rho(M)=$ $\max \left\{\left|\lambda_{1}\right|, \ldots,\left|\lambda_{n}\right|\right\}$, onde $\lambda_{i}$ são seus auto-valores. O raio espectral de uma matriz intervalar $\boldsymbol{M}$ é dado por

$$
\rho(\boldsymbol{M})=\max _{M \in \boldsymbol{M}} \rho(M) .
$$

Segundo Hansen e Walster (2004) o método de Gauss-Seidel intervalar é convergente se $\rho(|\boldsymbol{M}|)<1$, onde $|\boldsymbol{M}|$ é a matriz cujas entradas são $\left\|\boldsymbol{m}_{\boldsymbol{i} \boldsymbol{j}}\right\|$. 
Considere o sistema modificado

$$
B \boldsymbol{A}(\boldsymbol{y}-\operatorname{mid}(\boldsymbol{x}))=B \boldsymbol{b},
$$

onde $B=\operatorname{mid}(\boldsymbol{A})^{-1}$. Segundo Hansen e Walster (2004) e Kearfott (1996), esse sistema em geral é melhor condicionado do que o original. Em nossa implementação do método de Gauss-Seidel intervalar, adotamos esse pré-condicionamento. Usamos a biblioteca de álgebra linear Armadillo Sanderson (2010) para calcular $\operatorname{mid}(\boldsymbol{A})^{-1}$.

\subsection{Exemplo - Otimização Global}

Concluímos o capítulo mostrando os primeiros passos do interval branch and bound, aplicado a um problema de otimização irrestrito. Considere a função $f: \mathbb{R}^{2} \rightarrow \mathbb{R}$, dada por

$$
f\left(x_{1}, x_{2}\right)=\left(x_{1}^{2}+x_{2}^{2}-1\right)^{2}+\left(x_{1}^{2}-x_{2}^{2}\right) .
$$

Queremos resolver $\min _{x \in \boldsymbol{x}} f(x)$ na caixa $\boldsymbol{x}=([0,5],[0,5])$. Esse problema tem um única solução, o ponto $x^{*}=(0,1.2247)$. Veja a figura abaixo.

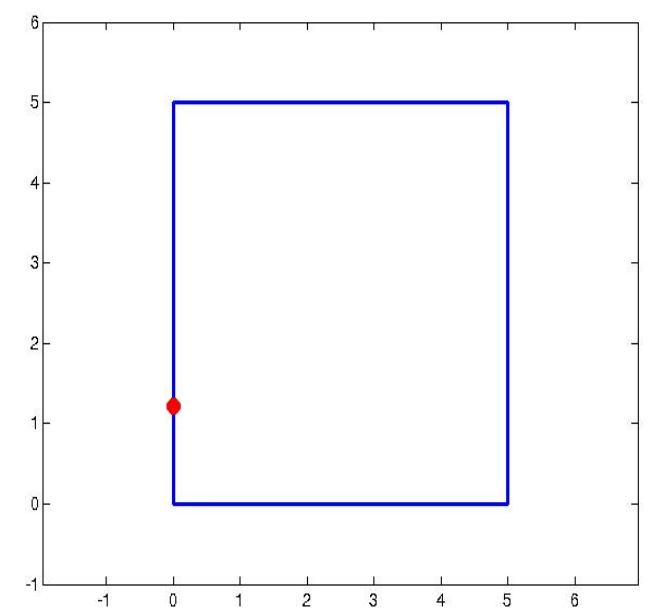

Figura 2.3: Exemplo do interval branch and bound - Inicio do Algoritmo

No início do algoritmo de Moore-Skelboe, avaliamos a função em $\boldsymbol{x}$ com aritmética intervalar

$$
\boldsymbol{f}(\boldsymbol{x})=[-250,2426]
$$

Como o cálculo é rigoroso, garantimos que $l b=-250$ é um limitante inferior do mínimo global. Para obter um limitante superior, basta avaliar $\boldsymbol{f}(\operatorname{mid}(\boldsymbol{x}))$, que nos dá $U b=132.5$.

Como a distância entre os limitantes é grande, dividimos $\boldsymbol{x}$ em duas para obter

$$
\begin{array}{lll}
\boldsymbol{x}_{\mathbf{1}}=([0,2.5],[0,5]) & \mathrm{com} & \boldsymbol{f}\left(\boldsymbol{x}_{\mathbf{1}}\right)=[-25,921.3126] \mathrm{e} \\
\boldsymbol{x}_{\mathbf{2}}=([2.5,5],[0,5]) & \mathrm{com} & \boldsymbol{f}\left(\boldsymbol{x}_{\mathbf{2}}\right)=[8.8,2426] .
\end{array}
$$

Uma vez que a caixa com menor limitante inferior é $\boldsymbol{x}_{1}$, ela será a próxima a ser processada. Guardamos a caixa $\boldsymbol{x}_{2}$ na lista de processamento junto com a informação de seu limitante inferior. A cada iteração escolhemos a caixa com menor limitante inferior para ser processada. 


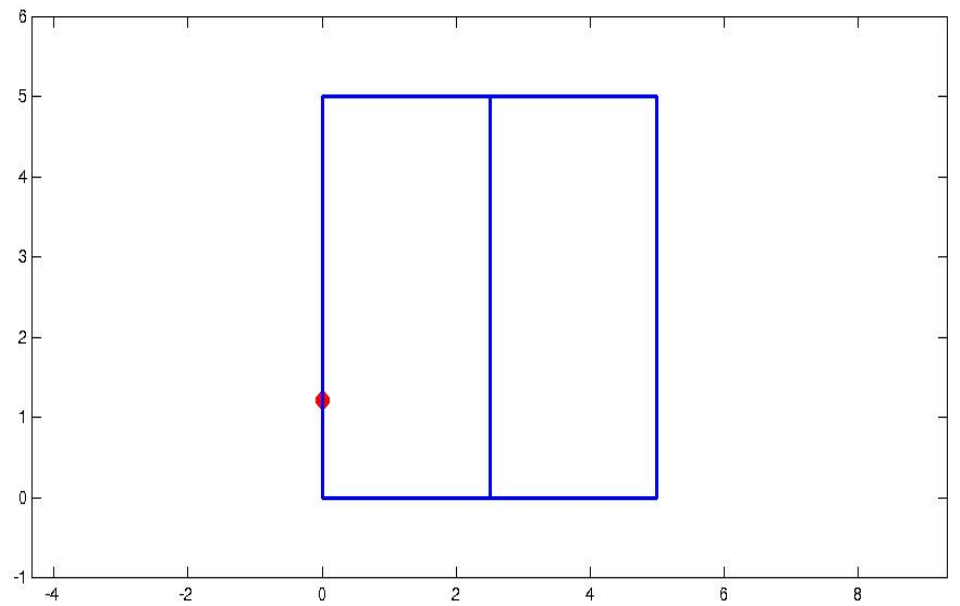

Figura 2.4: Exemplo do interval branch and bound - Primeira iteração

Caso alguma caixa satisfaça $\sup (\boldsymbol{x})-l b<\epsilon_{F}$ onde $\epsilon_{F}$ então a guardamos como solução. $\mathrm{O}$ algoritmo continua enquanto houver caixas para processar. É fácil mostrar que ele termina em um número finito de passos. A figura abaixo ilustra como excluímos caixas da lista de processamento.

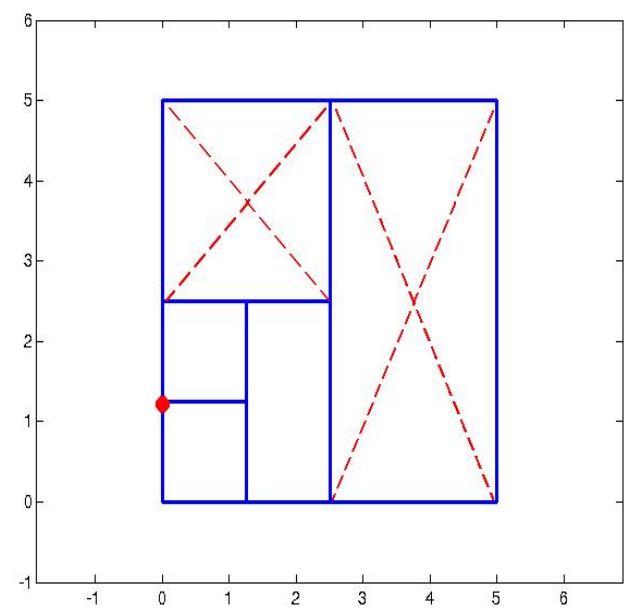

Figura 2.5: Exemplo do interval branch and bound - Quarta iteração

Neste caso, a caixa que estamos processando é $\boldsymbol{x}=([0,1.25],[0,1.25])$, que nos dá $\boldsymbol{f}(\boldsymbol{x})=$ $[-1.5626,6.0782]$. Observe que

$$
\sup (\boldsymbol{f}(\boldsymbol{x}))<\inf \left(\boldsymbol{f}\left(\boldsymbol{x}_{\mathbf{2}}\right) .\right.
$$

Sendo assim, $\boldsymbol{x}_{\mathbf{2}}$ não contém mínimos globais de $f$, e podemos excluí-la da busca. 


\section{Capítulo 3}

\section{Modelos de Markov Ocultos}

Conforme indicamos na introdução, um modelo de Markov oculto (MMO) é composto por dois processos estocásticos. O primeiro é uma cadeia de Markov, que não observamos diretamente. $\mathrm{O}$ segundo é um modelo de mistura observável, cuja distribuição depende do estado oculto. Neste capítulo apresentamos esses dois processos.

Modelos de Markov ocultos evoluíram ao longo do tempo para englobar um número maior de aplicações e aspectos teóricos. Os primeiros trabalhos, de Baum e Petrie (1966) e Baum e Eagon (1967) consideram modelos discretos no tempo, onde a cadeia de Markov e a mistura assumem um número finito de estados. Recentemente, Cappé et al. (2005) define os MMOs de forma bastante abrangente. Ele permite processos contínuos no tempo ou que assumem valores em espaços mais gerais.

Os resultados de nosso trabalho valem para modelos mais simples, como os definidos por Baum e seus colaboradores. Neste capítulo apresentamos os casos de interesse e definimos os MMOs intervalares. Nossos objetivos são

1. Revisar as duas componentes dos MMOs,

2. Introduzir modelos de Markov ocultos intervalares,

3. Limitar a caixa da distribuição estacionária em cadeias de Markov intervalares.

Quando a distribuição estacionária de uma cadeia de Markov é única, ela pode ser obtida pela solução de um sistema linear. Estendemos esse resultado para o caso intervalar e, a partir daí, comparamos a qualidade dos limitantes obtidos pelo método de Gauss-Seidel, descrito no capítulo anterior, com a função verifylss, descrita em Rump (1999).

O capítulo está dividido da seguinte forma. Na seção 3.1 apresentamos os modelos de mistura independente. Para mais detalhes sobre essa seção, consulte Zucchini e MacDonald (2009), Bulla et al. (2010) e Barbu et al. (2012). Na seção 3.2 introduzimos as cadeias de Markov. A principal referência dessa seção é o capítulo 15 de Feller (1968). A seção 3.3 define os modelos de Markov ocultos e sua versão intervalar. Concluímos o capítulo abordando a questão da distribuição estacionária em cadeias de Markov intervalares.

\subsection{Modelos de Mistura}

Considere o experimento onde temos duas variáveis aleatórias, $X_{1} \sim N(5,10)$ e $X_{2} \sim N(25,5)$. A cada momento, escolhemos uma delas para realizar uma observação. A escolha de qual variável será observada depende do lançamento de uma moeda $C \sim$ Bernoulli(0.25). A figura adaptada de Zucchini e MacDonald (2009) ilustra o experimento. 


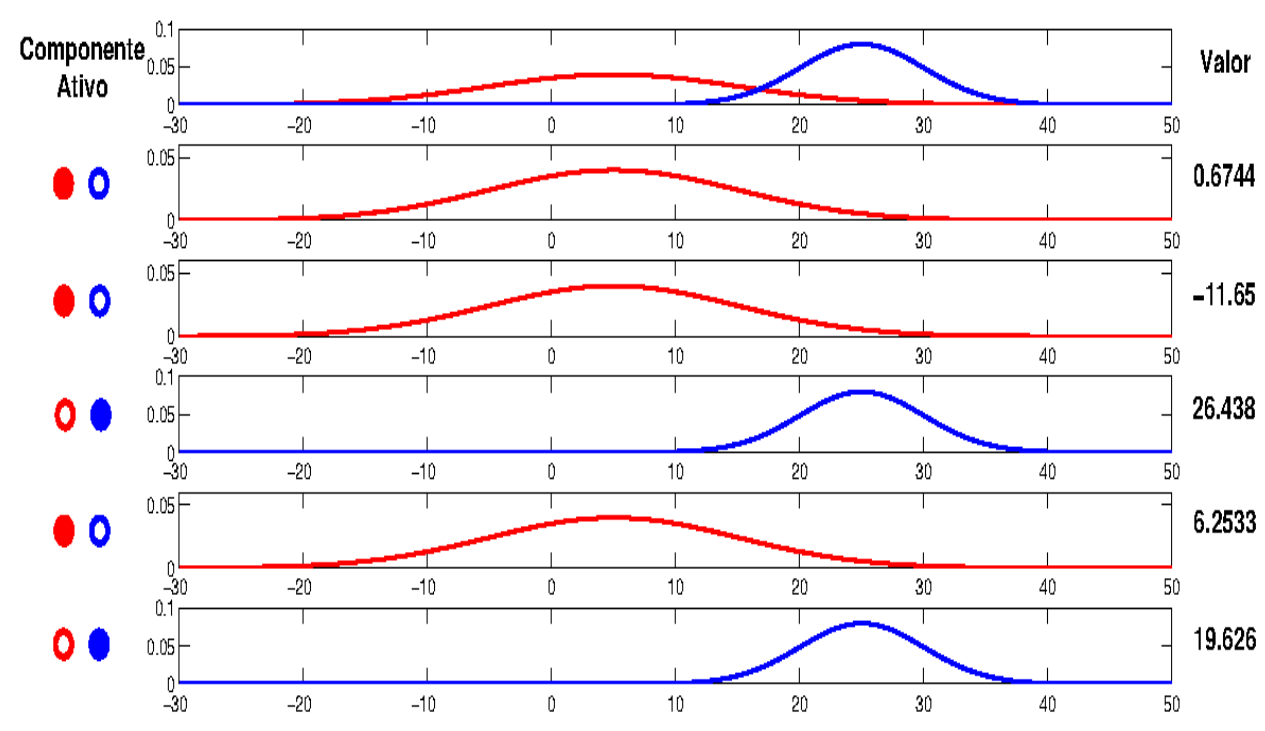

Figura 3.1: Realizações de um modelo de Markov oculto, com mistura normal. As componentes da mistura são $X_{1} \sim N(5,10)$ e $X_{2} \sim N(25,5)$. Na esquerda temos o componente escolhido a cada iteração, no meio sua função densidade e à direita o valor observado.

A cada momento observamos apenas a coluna valor e não sabemos se ele foi gerado por $X_{1}$ ou $X_{2}$. Ou seja, não conhecemos o resultado do lançamento da moeda. É claro que podemos generalizar o experimento. É possível tomar um número qualquer de variáveis aleatórias, com distribuições distinta.

Definição 7 Sejam $X_{1}, \ldots, X_{N}$ variáveis aleatórias tais que $X_{i} \sim \operatorname{Dist}\left(\theta_{i}\right)$. Considere ainda que $p\left(x ; \theta_{i}\right)$ é a função densidade ou de probabilidade de $X_{i}$. A distribuição p é uma mistura independente das $N$ variáveis se

$$
\begin{aligned}
p(x) & =\sum_{i=1}^{N} \lambda_{i} p\left(x ; \theta_{i}\right) \\
\sum_{i=1}^{N} \lambda_{i} & =1 \\
\lambda_{i} & \geq 0 .
\end{aligned}
$$

Dado um conjunto de observações $x_{1}, \ldots, x_{T}$, a estimação dos parâmetros $\lambda_{1}, \ldots, \lambda_{N}, \theta_{1}, \ldots, \theta_{N}$ segundo o princípio da verossimilhança se dá pela maximização da função

$$
L\left(\lambda_{1}, \theta_{1}, \ldots, \lambda_{N}, \theta_{N}, x_{1}, \ldots, x_{T}\right)=\prod_{i=1}^{T} \sum_{j=0}^{N} \lambda_{j} p_{j}\left(x_{i}, \theta_{j}\right) .
$$

As variáveis de decisão são $\lambda_{j}$ e $\theta_{i}$. Dessa forma, temos o problema de otimização a seguir

$$
\begin{aligned}
\max z & =L\left(\lambda_{1}, \theta_{1}, \ldots, \lambda_{N}, \theta_{N}, x_{1}, \ldots, x_{T}\right) \\
\theta_{i} & \in \Theta_{i} \\
\sum_{i=1}^{N} \lambda_{i} & =1 . \\
\lambda_{i} & \geq 0 .
\end{aligned}
$$


onde $\Theta_{i}$ é o espaço paramétrico do vetor de parâmetros $\theta_{i}$. Para evitar problemas de underflow, diversos autores sugerem a otimização de $\log (L()$.$) . Sendo assim, a função acima se torna o somatório$

$$
\log \left(L\left(\lambda_{1}, \theta_{1}, \ldots, \lambda_{N}, \theta_{N}, x_{1}, \ldots, x_{T}\right)\right)=\sum_{i=1}^{T} \log \left(\sum_{j=0}^{N} \lambda_{j} p_{j}\left(x_{i}, \theta_{j}\right)\right) .
$$

Este é um problema de otimização não convexo para a maioria das distribuições usadas em estatística. Como tal, ele pode ter vários máximos locais e não há solução analítica conhecida.

Para resolver problemas dessa forma, usamos algoritmos como o EM Dempster et al. (1977) ou métodos tradicionais de otimização, tais como os descritos em Nocedal e Wright (2006) e Floudas (2000).

Algoritmos locais, como o IPOPT Wächter e Biegler (2006) e o ALGENCAN Andreani et al. (2007) e Andreani et al. (2008), tendem a dar bons resultados, no entanto eles não garantem encontrar máximos globais. Se esse é o objetivo, consulte o código comercial BARON, descrito por Sahinidis (2014) ou o INTSOLVER, implementado durante nosso trabalho de mestrado.

Nesse trabalho, estamos interessados em modelos com mistura finita. Nesse caso, cada $X_{i}$ assume valores no conjunto $1, \ldots, M$. A distribuição das variáveis é dada pela matriz

$$
B=\left(\begin{array}{ccc}
b_{11} & \ldots & b_{1 M} \\
\vdots & \vdots & \vdots \\
b_{N 1} & \ldots & b_{N M}
\end{array}\right)
$$

Por questões históricas, $B$ é chamada matriz de emissão, veja Rabiner (1990). As linhas de $B$ são distribuições, isto é, elas satisfazem

$$
\sum_{j=1}^{M} b_{i j}=1 \quad i=1, \ldots, N .
$$

Os modelos de mistura que apresentamos são independentes. A escolha do modelo que irá gerar a próxima observação não depende de resultados anteriores. Os modelos de Markov ocultos generalizam esse caso. Eles expressam situações de dependência nos lançamentos da moeda. A forma mais simples de introduzir dependência é através das cadeias de Markov. Estudamos esses processos na próxima seção.

\subsection{Cadeias de Markov}

Considere o processo estocástico $\left(q_{n}\right)_{n \in \mathbb{N}} \operatorname{com}$ estados $1, \ldots, N$ e $N<\infty$. Diremos que $q_{n}$ tem propriedade de Markov se

$$
\mathbb{P}\left(q_{n}=j \mid q_{t-1}=i, \ldots\right)=\mathbb{P}\left(q_{t}=j \mid q_{t-1}=i\right)=a_{i j} .
$$

Claramente podemos agrupar os elementos $a_{i j}$ na matriz $A$, que será chamada de transição. Toda linha de $A$ é uma distribuição sobre $1, \ldots, N$. Isto é,

$$
\sum_{j=1}^{N} a_{i j}=1 \quad \forall i=1, \ldots, N .
$$

Uma cadeia de Markov é o par $(A, \pi)$, onde $A$ é a matriz de transição e $\pi$, o vetor de distribuição inicial. Nossa definição é bastante limitada. Ela não contempla processos como o passeio aleatório irrestrito, que tem um número infinito de estados, ou aqueles onde a transição ocorre em momentos aleatórios, como os processos de salto. Para uma apresentação detalhada e geral das cadeias de Markov, consulte Norris (1998). 
Definição 8 Chamamos de $p_{i j}^{n}$, a probabilidade de atingir o estado $j$ a partir de $i$ em n passos.

O cálculo de $p_{i j}^{n}$ se dá pela soma das probabilidades dos caminhos $\left(i, i_{1}, \ldots, i_{n-1}, j\right)$. Em particular, $p_{i j}^{1}=a_{i j} \mathrm{e}$

$$
p_{i j}^{2}=\sum_{k} a_{i k} a_{k j}
$$

Aplicando indução em $n$ temos

$$
p_{i j}^{n+1}=\sum_{k} a_{i k} a_{k j}^{n} .
$$

Definição 9 O estado $i$ tem período $t>1$ se $p_{i i}^{n}=0$, a menos que $n$ seja múltiplo de $t$ e $t$ seja o maior inteiro com essa propriedade.

Definição 10 A probabilidade de um caminho começando em $i$, atingir $j$ pela primeira vez no $n$-ésimo passo, é dada por $f_{i j}^{n}$.

Assim, $f_{i j}:=\sum_{n=1}^{\infty} f_{i j}^{n}$ nos dá a probabilidade do estado $i$ alcançar $j$ em algum momento. O estado $j$ é persistente se $f_{j j}=1$. Um estado que é aperiódico e persistente será chamado de ergódico. Uma cadeia onde todos os estados são ergódicos também recebe este nome.

Definição 11 A distribuição estacionária da matriz de transição A é um vetor $\delta$ com entradas não negativas e tal que

$$
\begin{aligned}
A^{\prime} \delta & =\delta \\
\sum_{i=1}^{N} \delta_{i} & =1 .
\end{aligned}
$$

Por exemplo, a matriz

$$
A=\left(\begin{array}{ccc}
\frac{1}{3} & \frac{1}{3} & \frac{1}{3} \\
\frac{2}{3} & 0 & \frac{1}{3} \\
\frac{1}{2} & \frac{1}{2} & 0
\end{array}\right)
$$

tem como distribuição estacionária o vetor $\delta^{T}=\frac{1}{32}(15,9,8)$.

Uma cadeia de Markov é irredutível se há um caminho ligando dois estados quaisquer. Feller (1968) mostra o resultado a seguir

Teorema 3 Uma cadeia irredutivel onde todos os estados são ergódicos admite uma e somente uma distribuição estacionária.

Há várias formas de calcular a distribuição estacionária da uma cadeia de Markov. Em nosso trabalho, usamos um resultado demonstrado em Zucchini e MacDonald (2009).

Teorema 4 Seja $(A, \pi)$ uma cadeia de Markov com $N$ estados. O vetor não negativo $\delta$ é distribuição estacionária de $A$ se e somente se

$$
\left(I-A^{T}+U\right) \delta=1
$$

onde I é a matriz identidade de dimensão $N, 1$ é o vetor unitário e $U=1^{\prime} 1$.

No final do capítulo, usamos esse teorema para limitar a distribuição estacionária de uma matriz intervalar $\boldsymbol{A}$.

Ao longo do texto, assumimos que as cadeias são ergódicas, a menos de menção contrárias. Rabiner (1990) nos dá exemplo de cadeias não ergódicas com aplicações ao reconhecimento de voz. É o caso das cadeias left-right, cuja matriz de transição é da forma

$$
A=\left(\begin{array}{cccc}
a_{11} & a_{12} & \ldots & a_{1 N} \\
0 & a_{22} & \ldots & a_{2 N} \\
\vdots & \vdots & \vdots & \vdots \\
0 & 0 & \ldots & 1
\end{array}\right)
$$


Cadeias com estrutura especial permitem eliminar variáveis de decisão durante o interval branch and bound. Essa redução de variáveis torna o algoritmo mais eficiente. Nossa implementação permite lidar com cadeias ergódicas ou estruturadas. No último caso, basta fixar o valor do parâmetro antes de começar a otimização.

\subsection{Modelos de Markov Ocultos}

Todo MMO é composto por uma cadeia de Markov e um modelo de mistura. Na seção anterior, dissemos que este trabalho considera apenas cadeias de Markov discretas no tempo e com número finito de estados. Agora, formalizamos o conceito de modelo de Markov oculto e definimos sua forma intervalar.

\subsubsection{Definição}

Sejam $(A, \pi)$ uma cadeia de Markov com $N$ estados e $X_{1}, \ldots, X_{N}$, variáveis aleatórias sobre o conjunto $\{1, \ldots, M\}$. A probabilidade de observarmos o simbolo $j$ com a variável $X_{i}$ é $\mathbb{P}\left(X_{i}=j\right)=$ $b_{i j}$. Agrupando essas probabilidade temos a matriz de emissão $B$.

Definição 12 Um modelo de Markov oculto é a tripla $\theta=(A, B, \pi)$. O MMO cuja matriz de transição tem $N$ estados e a mistura assume $M$ símbolos é chamado de $M M O_{N, M}$.

O resultado de $T$ realizações de um modelo de Markov oculto é o par $(o, q)$. Aqui, $o=\left(o_{1}, \ldots, o_{T}\right)$ é o conjunto de símbolos observados e $q=\left(q_{1}, \ldots, q_{T}\right)$ são os estados da cadeia de Markov, que não observamos.

Um caso particular importante é o $M M O_{2,2}$ que chamamos de modelos de Markov ocultos com mistura de Bernoulli. Eles são parametrizados por

$$
A=\left(\begin{array}{ll}
a_{11} & a_{12} \\
a_{21} & a_{22}
\end{array}\right) \quad B=\left(\begin{array}{ll}
b_{11} & b_{12} \\
b_{21} & b_{22}
\end{array}\right) \quad \pi=\left(\begin{array}{c}
\pi_{1} \\
\pi_{2}
\end{array}\right) .
$$

Lembrando que as linhas da matriz de emissão são distribuições, temos $b_{11}+b_{12}=1$ e $b_{21}+b_{22}=1$. Dessa forma, escrevemos $b_{11}=1-b_{12}$ e $b_{22}=1-b_{21}$. Essa representação permite visualizar propriedades desses modelos através de gráficos com eixos $b_{11}$ e $b_{22}$. Por essa razão, a maior parte dos experimentos que realizamos nos próximos capítulos consideram o $M M O_{2,2}$.

\subsubsection{Modelos de Markov Ocultos Intervalares}

Em um modelo de Markov oculto intervalar, as probabilidades da cadeia de Markov ou do modelo de mistura não são conhecidos exatamente. Sabemos apenas que elas pertencem a determinados intervalos. Segundo as notações estabelecidas na seção anterior e no capítulo 2, um modelo de Markov oculto intervalar é parametrizado pela tripla

$$
\boldsymbol{\theta}=(\boldsymbol{A}, \boldsymbol{B}, \boldsymbol{\pi}) .
$$

Por exemplo, considere $\boldsymbol{\theta}=(\boldsymbol{A}, \boldsymbol{B}, \boldsymbol{\pi})$ onde

$$
\boldsymbol{A}=\left(\begin{array}{cc}
{\left[\frac{1}{3}, \frac{2}{3}\right]} & {\left[\frac{1}{4}, \frac{3}{4}\right]} \\
\frac{4}{5} & \frac{1}{5}
\end{array}\right), \pi=\left(\begin{array}{c}
{[0.32,0.67]} \\
{[0.25,0.75]}
\end{array}\right)
$$

e

$$
\boldsymbol{B}=\left(\begin{array}{cc}
{\left[\frac{1}{4}, \frac{2}{3}\right]\left[\frac{1}{10}, \frac{1}{5}\right]\left[\frac{1}{4}, \frac{3}{4}\right]} \\
\frac{1}{4} \quad\left[\frac{1}{4}, \frac{2}{3}\right]\left[\frac{1}{10}, \frac{1}{5}\right]
\end{array}\right)
$$


A tripla $\boldsymbol{\theta}$ parametriza um modelo de Markov oculto intervalar. Assim como as linhas de $A, B$ e $\pi$ devem ser distribuições, para que $\boldsymbol{\theta}$ seja uma parametrização ele deve satisfazer

$$
\begin{array}{rlr}
1 & \in \boldsymbol{a}_{\boldsymbol{i} \mathbf{1}}+\ldots \boldsymbol{a}_{\boldsymbol{i N}} . & i=1, \ldots, N . \\
1 & \in \boldsymbol{b}_{\boldsymbol{i 1}}+\ldots+\boldsymbol{b}_{\boldsymbol{i} \boldsymbol{M}}, \quad i=1, \ldots, N . \\
1 & \in \boldsymbol{\pi}_{\mathbf{1}}+\ldots+\boldsymbol{\pi}_{\boldsymbol{N}}, \\
\boldsymbol{a}_{\boldsymbol{i j}}, \boldsymbol{b}_{\boldsymbol{i} \boldsymbol{j}}, \boldsymbol{\pi}_{\boldsymbol{i}} & \subseteq[0,1] .
\end{array}
$$

Se essas condições não são satisfeitas, diremos que os parâmetros são inconsistentes.

Essas relações permitem obter cadeias intervalares estreitas. Por exemplo, observe o vetor $\boldsymbol{\pi}$ acima. É claro que se $\pi_{1} \geq \frac{1}{3}$ implica que $\pi_{2}<\frac{3}{4}$. Portanto, o limitante superior de $\boldsymbol{\pi}_{\mathbf{2}}$ está sobrestimado. O procedimento abaixo, conhecido como propagação de restrições Neumaier (2004), permite estreitar os intervalos dos parâmetros. Considere o sistema

$$
\begin{aligned}
\pi_{1}+\ldots+\pi_{N} & =1 \\
\pi_{i} & \in \boldsymbol{\pi}_{\boldsymbol{i}} .
\end{aligned}
$$

É claro que $\pi_{i}=1-\sum_{\substack{j=1 \\ j \neq i}} \pi_{j}$. Pelo teorema fundamental da aritmética intervalar, todos os pontos que satisfazem o sistema pertencem a $\boldsymbol{\pi}_{\boldsymbol{i}} \cap \mathbf{1}-\sum_{\substack{j=1 \\ j \neq i}} \boldsymbol{\pi}_{\boldsymbol{j}}$. Sendo assim escrevemos

$$
\boldsymbol{\pi}_{\boldsymbol{i}}{ }^{*}=\left(1-\sum_{\substack{j=1 \\ j \neq i}} \boldsymbol{\pi}_{\boldsymbol{j}}\right) \cap \boldsymbol{\pi}_{\boldsymbol{i}} .
$$

Caso a interseç̧ão seja vazia, provamos que o sistema não tem solução, ou seja não existem $\pi_{1} \in$ $\pi_{1}, \ldots, \pi_{N} \in \pi_{N}$ tais que $\pi_{1}+\ldots+\pi_{N}=1$. Aplicando essa relação ao exemplo, temos

$$
\pi_{1}^{*}=\left(1-\left[\frac{1}{4}, \frac{3}{4}\right]\right) \cap\left[\frac{1}{3}, \frac{2}{3}\right]=\left[\frac{1}{3}, \frac{2}{3}\right]
$$

e

$$
\boldsymbol{\pi}_{\mathbf{2}}{ }^{*}=\left(1-\left[\frac{1}{3}, \frac{2}{3}\right]\right) \cap\left[\frac{1}{4}, \frac{3}{4}\right]=\left[\frac{1}{3}, \frac{2}{3}\right] .
$$

Nesse caso, não melhoramos o intervalo $\boldsymbol{\pi}_{1}$, mas estreitamos $\boldsymbol{\pi}_{2}$ e $\boldsymbol{\pi}^{*}$ é um modelo mais estreito que $\boldsymbol{\theta}$. Podemos repetir a operação enquanto houver decréscimo nas caixas $\boldsymbol{\pi}_{\mathbf{1}}$ ou $\boldsymbol{\pi}_{\mathbf{2}}$.

Dada uma caixa $\boldsymbol{x}=\left(\boldsymbol{x}_{1}, \ldots, \boldsymbol{x}_{\boldsymbol{N}}\right)$, o algoritmo Propagação linear das restrições, descrito no apêndice A devolve uma nova caixa $\boldsymbol{x}^{\prime}$ tal que $\boldsymbol{x}^{\prime} \subseteq \boldsymbol{x}$. O algoritmo também prova a inconsistência, caso $\boldsymbol{x}^{\prime}$ seja vazia.

\subsection{Distribuição Invariante Intervalar}

Dada uma matriz intervalar que satisfaz as equações (3.2) - (3.5), estamos interessados em limitar o casco intervalar do conjunto

$$
\left.\mathcal{S}:=\left\{x \in \mathbb{R}^{N} \mid \exists A \in \boldsymbol{A} \text { tal que }\left(I-A^{T}+U\right) x=1\right)\right\} .
$$

Comparamos agora duas formas de resolver esse problema. A primeira, através do algoritmo de Gauss-Seidel intervalar, que apresentamos apresentado na seção 2.3. A outra, através da função verifylss do INTLAB, descrita em Rump (2001).

Seja $A$ a matriz de transição real gerada aleatoriamente

$$
A=\left(\begin{array}{ll}
0.3067 & 0.6933 \\
0.6951 & 0.3049
\end{array}\right) \text {. }
$$


Construímos a figura abaixo a partir da matriz intervalar $\boldsymbol{A}=\operatorname{midrad}(A, 0.25)$. Isto é, cada entrada $\boldsymbol{a}_{i j}$ de $\boldsymbol{A}$ é um intervalo com centro $a_{i j}$ e raio 0.25 .

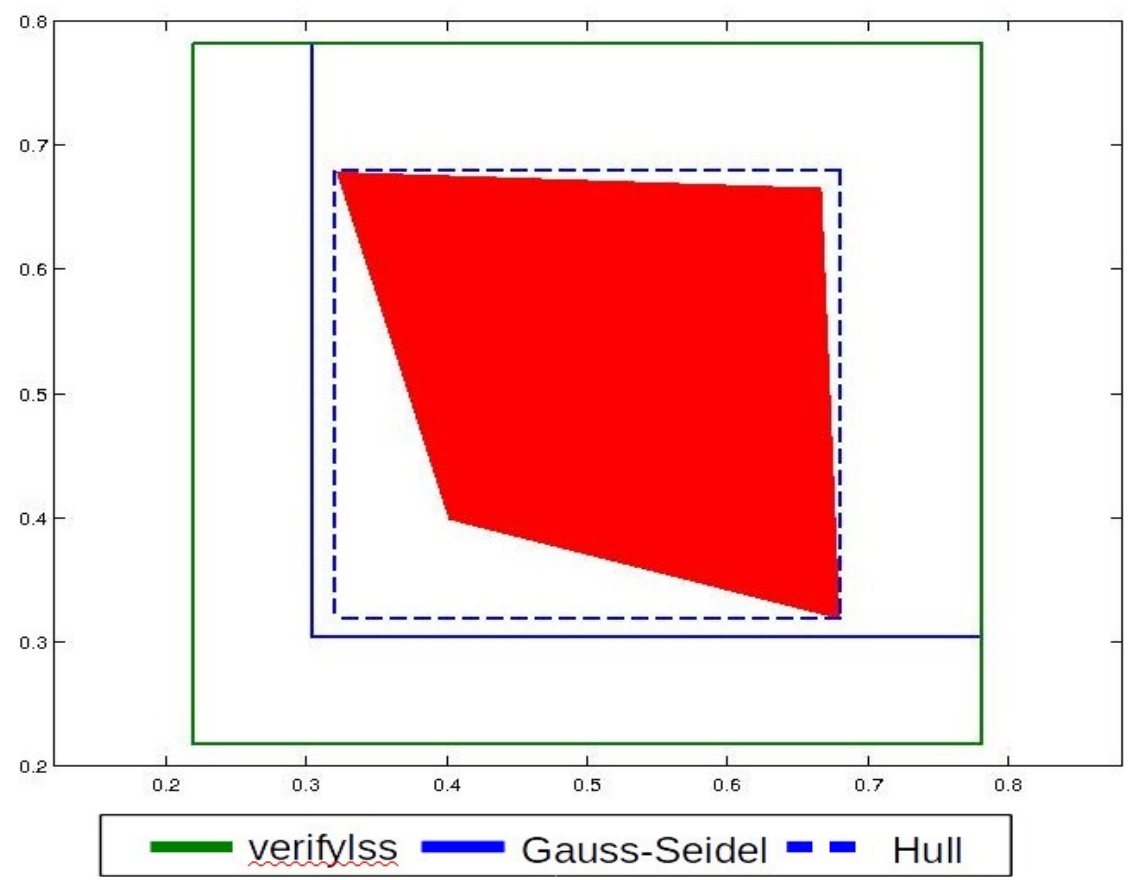

Figura 3.2: Comparação entre os algoritmos Gauss-Seidel intervalar e verifylss para limitar a solução de (3.4) $\operatorname{com} \boldsymbol{A}=\operatorname{midrad}(A, 0.25)$

Em vermelho, observamos o conjunto $\mathcal{S}$. A linha azul tracejada é o casco intervalar desse conjunto. Nenhum dos dois métodos foi capaz de determinar o casco intervalar exatamente No entanto, o algoritmo de Gauss-Seidel produziu uma caixa mais estreita. O intervalo inicial para o método de Gauss-Seidel nesse teste foi $x_{0}^{T}=([0,1],[0,1])$. A função verifylss não exige uma caixa inicial.

Considere agora a tabela que resume o mesmo experimento, com matrizes de dimensões diferentes.

\begin{tabular}{|c|c|c|c|c|}
\hline$N$ & $r$ & $\max (w(\boldsymbol{g s}(\boldsymbol{x})))$ & $\max (w(\boldsymbol{v e r i f y l s s}(\boldsymbol{x})))$ & $\mid$ col4 - col3 $\mid$ \\
\hline 2 & 0.1 & 0.429900 & 0.501713 & $7.181346 \mathrm{e}-02$ \\
\hline 2 & 0.01 & 0.033996 & 0.034564 & $5.678973 \mathrm{e}-04$ \\
\hline 3 & 0.01 & 0.043443 & 0.044362 & $9.181946 \mathrm{e}-04$ \\
\hline 3 & 0.001 & 0.004238 & 0.004247 & $8.955682 \mathrm{e}-06$ \\
\hline 5 & 0.01 & 0.052592 & 0.053880 & $1.287768 \mathrm{e}-03$ \\
\hline 5 & 0.001 & 0.004930 & 0.004942 & $1.207124 \mathrm{e}-05$ \\
\hline 10 & 0.01 & 0.047511 & 0.048455 & $9.440198 \mathrm{e}-04$ \\
\hline 10 & 0.001 & 0.004039 & 0.004047 & $8.024248 \mathrm{e}-06$ \\
\hline
\end{tabular}

Tabela 3.1: Comparação entre os algoritmos Gauss-Seidel intervalar e verifylss para limitar a solução de (3.4). A dimensão da matriz é $N$, $r$ é o raio da matriz $\boldsymbol{A}$

O método de Gauss-Seidel intervalar produz caixas mais estreitas. No entanto, essa vantagem diminui à medida que diminuímos $r$. Este comportamento é esperado pois $\boldsymbol{A} \rightarrow A$ conforme $r \rightarrow 0$. Ele indica que os dois métodos estão limitando corretamente $\mathcal{S}$.

De modo geral, é vantajoso usar o método de Gauss-Seidel intervalar devido à pequena vantagem nas caixas obtidas. Ambos os procedimentos são eficientes. Nenhum caso apresentado na tabela levou mais do que 0.5 segundos para ser resolvido. 


\section{Capítulo 4}

\section{Avaliando a Verossimilhança}

Neste capítulo estudamos o cálculo de probabilidades em MMOs reais e intervalares. Dado o modelo parametrizado por $\theta=(A, B, \pi)$ e o conjunto de observações $O=\left(o_{1}, o_{2}, \ldots, o_{T}\right)$, queremos avaliar $\mathbb{P}(O \mid \theta)$. Introduzimos também a função de verossimilhança $L_{O}(\theta)$ e suas derivadas de primeira e segunda ordem. Todos os cálculos são feitos com a recursão backward, descrita em Rabiner (1990).

Essa recursão exige uma série de produtos no intervalo $[0,1]$. Portanto, é necessário adotar estratégias que evitem aproximações indevidas para 0. Vários autores sugerem normalizar o resultado a cada iteração com a função logarítmica. Nós apresentamos uma alternativa eficiente, que pode ser facilmente estendida para o caso intervalar.

Sejam $\boldsymbol{\theta}=(\boldsymbol{A}, \boldsymbol{B}, \boldsymbol{\pi})$ parâmetros de um modelo oculto intervalar. Estamos interessados em limitar a variação $\overline{\boldsymbol{L}_{O}(\boldsymbol{\theta})}$. Para tanto, partimos da recursão backward e construímos sua extensão natural intervalar. Mostramos que a implementação ingênua dessa extensão nos dá limitantes grosseiros de $\overline{\mathbb{P}(O \mid \boldsymbol{\theta})}$, além de exigir um número de trocas no modo de arredondamento proporcional ao tamanho da amostra.

O principal resultado do capítulo é a introdução de uma nova extensão, baseada em programação linear. Nossa alternativa produz resultados mais estreitos e com número fixo de trocas no modo de arredondamento, inclusive para o cálculo das derivadas.

Ao longo do capítulo, a seqüência de estados ocultos é dada por $q=\left(q_{1}, \ldots, q_{T}\right)$. O modelo de Markov oculto é parametrizado por $\theta=(A, B, \pi)$. O par $(A, \pi)$ é uma cadeia de Markov com $N$ estados $1, \ldots, N$. O modelo de mistura assume valores no conjunto $\{1,2, \ldots, M\}$ e tem matriz de emissão dada por,

$$
B=\left(\begin{array}{ccc}
b_{11} & \ldots & b_{1 M} \\
\vdots & \vdots & \vdots \\
b_{N 1} & \ldots & b_{N M}
\end{array}\right)
$$

Além disso, as linhas de $A, B$ e $\pi$ são distribuições, isto é

$$
\begin{aligned}
& \sum_{j=1}^{N} a_{i j}=1 \quad i=1, \ldots, N . \\
& \sum_{j=1}^{M} b_{i j}=1 \quad i=1, \ldots, N . \\
& \sum_{j=1} \pi_{j}=1 .
\end{aligned}
$$


Escrevemos ainda $P\left(o_{n}\right)$ para indicar a matriz diagonal cujas entradas são $\left(b_{1 o_{n}}, \ldots, b_{N o_{n}}\right)$. Ou seja,

$$
P\left(o_{n}\right)=\left(\begin{array}{cccc}
b_{1 o_{n}} & 0 & \ldots & 0 \\
0 & b_{2 o_{n}} & \ldots & 0 \\
\vdots & \vdots & \vdots & \vdots \\
0 & 0 & \ldots & b_{N o_{n}}
\end{array}\right)
$$

Os modelos intervalares tem as mesmas componentes. Neles, dois ou mais parâmetros da tripla $\boldsymbol{\theta}=(\boldsymbol{A}, \boldsymbol{B}, \boldsymbol{\pi})$ não são conhecidos exatamente. Sabemos apenas que eles pertencem a determinados intervalos.

Esse capítulo está dividido da seguinte forma. Na seção 4.1 revisamos o caso real a partir das recursões forward-backward. Derivamos também as formulas do gradiente e hessiana de $L_{O}(\theta)$. A seção 4.2 discute a extensão natural intervalar da recursão backward. Na seção 4.3 mostramos nossa extensão intervalar, baseada em programação linear.

\subsection{Revisão do Caso Real}

Nesta seção revisamos as recursões forward-backward, que permitem o cálculo de probabilidades em MMOs. Discutimos o procedimento de normalização proposto na literatura e mostramos uma alternativa, que não depende do logaritmo. Apresentamos ainda a função de verossimilhança dos modelos de Markov ocultos. Concluímos derivando formulas para suas derivadas de primeira e segunda ordem.

\subsubsection{Recursões Forward-Backward}

Segundo a definição de modelo de Markov oculto, temos

$$
\mathbb{P}(O \mid \theta)=\sum_{\mathcal{S}} \mathbb{P}\left(O_{1}^{T} \mid q_{1}^{T}, \theta\right) * \mathbb{P}\left(q_{1}^{T} \mid \theta\right)
$$

Aqui, $\mathcal{S}:=\left\{q=\left(q_{1}, \ldots, q_{T}\right) \mid q_{n} \in\{1, \ldots N\}, n=1, \ldots, T\right\}$ é o conjunto de todas as seqüências de tamanho $T$, envolvendo $N$ elementos e com repetição. Expandindo o somatório, temos

$$
\mathbb{P}(O \mid \theta)=\sum_{\left(q_{1}, \ldots, q_{T}\right) \in \mathcal{S}} \pi_{q_{1}} b_{q_{1} o_{1}} a_{q_{1} q_{2}} b_{q_{2} o_{2}} \ldots a_{q_{T-1} q_{T}} b_{q_{T} o_{T}} .
$$

Essa equação deve ser lida da seguinte forma. No momento $n=1$, a probabilidade do estado oculto ser $q_{1}$ e, a partir dele, observarmos $o_{1}$ é $\pi_{q_{1}} b_{q_{1} o_{1}}$. Em seguida, transitamos de $q_{1}$ para $q_{2}$ e realizamos uma nova observação. A iteração se repete até atingir $T$ passos. Ao final, $\mathbb{P}(O \mid \theta)$ é a soma de todas as seqüências possíveis e com repetição de estados ocultos.

Embora $\mathcal{S}$ seja um conjunto finito, sua cardinalidade é $2^{N^{T}}$ e o cálculo acima requer $O\left(2 T * N^{T}\right)$ operações de ponto flutuante. É claro que esse procedimento é inviável. Por exemplo, se $N=2$ e $T=100$, são necessárias $O\left(10^{12}\right)$ operações de ponto flutuante para avaliar uma única probabilidade.

O estudo dos modelos de Markov ocultos só é possível a partir das recursões forward e backward, descritas pela primeira vez em Baum e Petrie (1966). Considere $\alpha$ e $\beta$ definidas a seguir

$$
\alpha_{n}(i)=\mathbb{P}\left(o_{1}, \ldots, o_{n}, q_{n}=i \mid \theta\right)
$$

e

$$
\beta_{n}(i)=\mathbb{P}\left(o_{n+1}, \ldots, o_{T} \mid q_{n}=i, \theta\right) .
$$


A recursão forward é dada por

$$
\begin{aligned}
\alpha_{1}(i) & =\pi_{i} p_{i}\left(o_{1}\right) \quad 1 \leq i \leq N, \\
\alpha_{n+1}(j) & =\sum_{i=1}^{N} \alpha_{n}(i) a_{i j} p_{j}\left(o_{n+1}\right) \quad n=1, \ldots, T-1 \text { e } 1 \leq j \leq N, \\
\mathbb{P}(O \mid \theta) & =\sum_{i=1}^{N} \alpha_{T}(i) .
\end{aligned}
$$

Essa recursão já nos dá $\mathbb{P}(O \mid \theta)$ e poderíamos usá-la na construção de nossos algoritmos. No entanto, a forma backward tem propriedades interessantes que favorecem seu uso. Segundo a definição de $\beta$

$$
\begin{aligned}
\beta_{T}(i) & =1 \\
\beta_{n}(i) & =\sum_{j=1}^{N} a_{i j} p_{j}\left(o_{n+1}\right) \beta_{n+1}(i) \quad n=T-1, \ldots, 1 \text { e } 1 \leq i \leq N, \\
\mathbb{P}(O \mid \theta) & =\sum_{i=1}^{N} \pi_{i} p_{i}\left(o_{1}\right) \beta_{1}(i) .
\end{aligned}
$$

As recursões têm uma forma matricial, tal como em Levinson et al. (1983). Temos,

$$
\begin{aligned}
\alpha_{1} & =\pi^{\prime} P\left(o_{1}\right), \\
\alpha_{n+1} & =\alpha_{n} A P\left(o_{n+1}\right) \quad n=1, \ldots, T-1, \\
\mathbb{P}(O \mid \theta) & =\sum_{i=1}^{N} \alpha_{T}(i)
\end{aligned}
$$

e

$$
\begin{aligned}
\beta_{T} & =\overrightarrow{1} \\
\beta_{n} & =A P\left(o_{n+1}\right) \beta_{n+1} \quad n=T-1, \ldots, 1, \\
\mathbb{P}(O \mid \theta) & =\pi^{\prime} P\left(o_{1}\right) \beta_{1} .
\end{aligned}
$$

Rabiner (1990) afirma que usando qualquer uma das recursões, são necessários $N(N+1)(T-1)+N$ produtos e $N(N-1)(T-1)$ somas para avaliar $\mathbb{P}(O \mid \theta)$. Dessa forma, o número de operações necessárias para calcular uma probabilidade é linear em $T$ e não mais exponencial. No exemplo acima, seriam necessárias 1190 operações de ponto flutuante contra as $10^{12}$ do parágrafo anterior, um ganho na ordem de um bilhão.

\subsubsection{Normalização}

O cálculo de $\mathbb{P}(O \mid \theta)$ exige uma série de produtos com valores entre 0 e 1 . Portanto, temos de lidar com problemas de underflow. Zucchini e MacDonald (2009), Bulla et al. (2010) e Cappé et al. (2005) sugerem normalizar os vetores $\alpha$ e $\beta$ com a função logarítmica. Para tanto, são necessárias as variáveis auxiliares $\phi_{n}$ e $w_{n}$, dadas por

$$
\begin{aligned}
w_{n} & =\sum_{i=1}^{N} \beta_{n}(i), \\
\phi_{n} & =\frac{\beta_{n}}{w_{n}} .
\end{aligned}
$$

A cada observação, normalizamos o vetor $\beta_{n}$ a partir da soma de suas componentes. Outro procedimento possível é tomar $w_{n}=\max \left\{\beta_{1}(i), \ldots, \beta_{N}(i)\right\}$ ao invés da soma. $\mathrm{O}$ algoritmo abaixo calcula 
$\log \left(\mathbb{P}\left(O_{1}^{T} \mid \theta\right)\right)$ usando a recursão backward.

Require: Os parâmetros $\theta$ e o conjunto de observações $O$.

Ensure: $\log (\mathbb{P}(O \mid \theta))$

1: function LOG_ $\beta(\theta, O)$

2: $\quad \phi_{T} \leftarrow \overrightarrow{\mathbf{1}}$

3: $\quad l \leftarrow 0$;

4: $\quad$ for $n=T-1, T-2, \ldots, 1$ do

5: $\quad v \leftarrow A P\left(o_{n}\right) \phi_{n+1}$

6: $\quad u \leftarrow v^{\prime} \overrightarrow{1}$

7: $\quad l \leftarrow l+\log (u)$;

8: $\quad \phi_{n}=\frac{v}{u}$;

9: $\quad$ end for

10: $\quad v \leftarrow \pi^{\prime} P\left(o_{1}\right) \phi_{1}$

11: $\quad u \leftarrow v^{\prime} \overrightarrow{1}$;

12: $\quad l \leftarrow l+\log (u)$;

13: $\quad$ return $l$;

\section{4: end function}

É claro que podemos redefinir as variáveis auxiliares e o algoritmo para aplicar a recursão forward. O cálculo de $\log (\mathbb{P}(O \mid \theta))$ é comum em estatística mas devemos evitá-lo quando lidamos com aritmética intervalar. Dado o intervalo $\boldsymbol{x}$, a extensão natural do logaritmo é

$$
\log (\boldsymbol{x})=[\nabla(\log (\inf (\boldsymbol{x}))), \Delta(\log (\sup (\boldsymbol{x})))],
$$

onde $\nabla$ e $\triangle$ indicam as direções do arredondamento. Infelizmente, as instruções para o cálculo do $\log$ não respeitam o modo de arredondamento nos processadores atuais. Portanto, para garantir o rigor do cálculo, recorremos à bibliotecas que realizem esta operação. A $M P F R$, descrita em Fousse et al. (2007) é uma dessas bibliotecas. A figura abaixo compara os tempos entre as funções $\log$ da biblioteca cmath.h e da MPFR. O teste foi realizado em um processador core-ir, com o compilador $i c c++14.0 .2$ e opções de compilação -O3, -frounding-math e -NDEBUG.

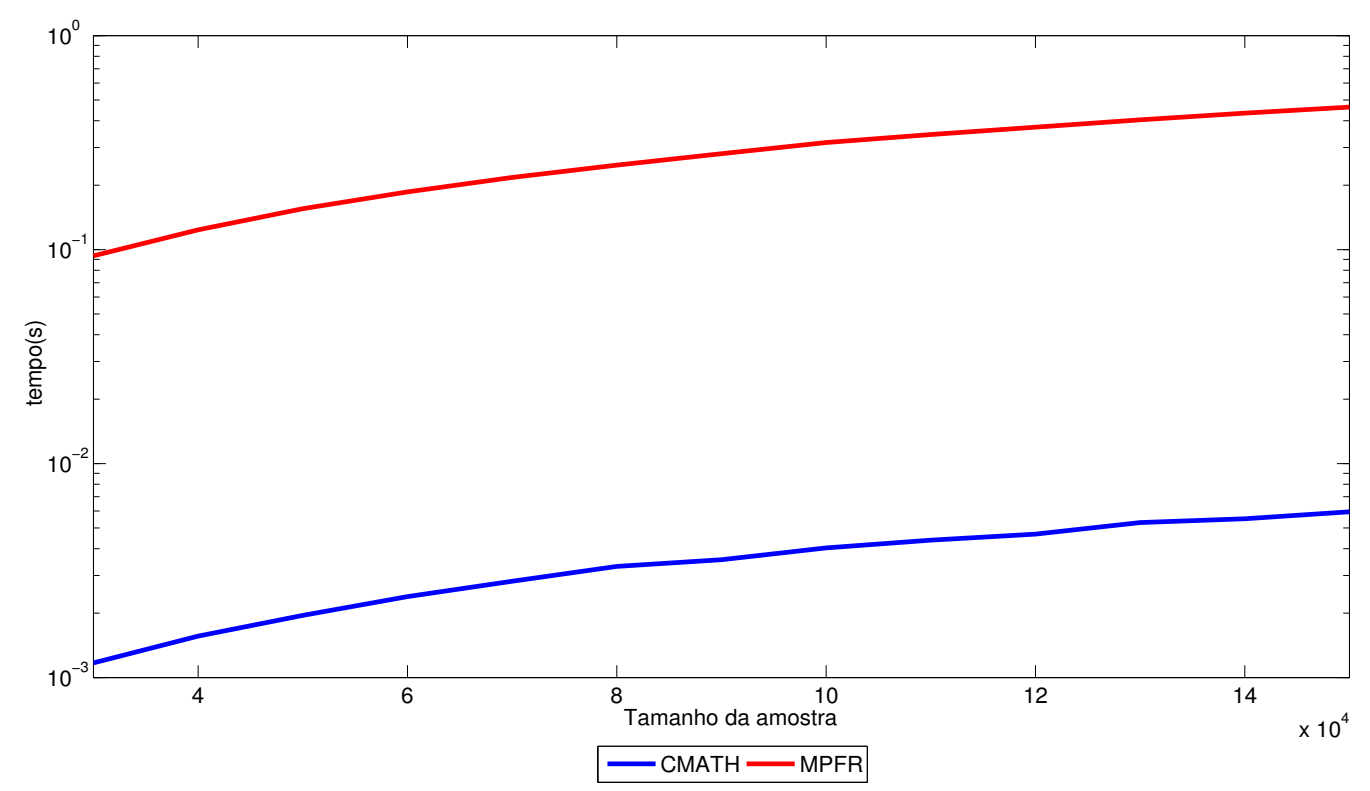

Figura 4.1: Cálculo do logaritmo realizado com a função padrão da cmath.h e com a biblioteca MPFR, especificando o modo de arredondamento para baixo. Amostras geradas aleatoriamente no intervalo $[0,1]$. 
O experimento mostra que uma avaliação de $\log$ com a $M P F R$, é cerca de 100 vezes mais lenta do que com a biblioteca padrão. Precisamos então de uma alternativa de normalização que seja eficiente quando realizada em intervalos.

Nossa solução é inspirada na aritmética de ponto flutuante. Um número dessa forma é composto de três partes, o sinal, a mantissa e o expoente. Por exemplo, um número do tipo double é um conjunto de 64 bits, onde o primeiro guarda o sinal, os próximos 11 expressam o expoente e os demais a mantissa.

Em nossa implementação construímos uma classe de números normalizados. Um número normalizado é composto de duas partes, a mantissa e o expoente. O primeiro é um número de ponto flutuante e o último, um inteiro. Dessa forma, temos 32 bits para guardar o expoente e não apenas 11. O número normalizado resolve o problema de underflow em todos os casos de interesse. Ele também não tem um campo de sinal, pois a mantissa é capaz de armazenar valores positivos ou negativos.

Na construção dessa classe usamos duas funções da biblioteca cmath do $C++$. A primeira é a frexp, que recebe um número de ponto flutuante e devolve seu expoente e mantissa. A segunda é a ldexp, que recebe um número de ponto flutuante $v$, um inteiro exp e calcula $v * 2^{\text {exp }}$. Nosso algoritmo para calcular $\mathbb{P}(O \mid \theta)$ é dado abaixo.

Require: Os parâmetro $\theta$ e o conjunto de observações $O$.

Ensure: O inteiro exp e o número de ponto flutuante $s$ tais que $s * 2^{\exp }=\mathbb{P}(O \mid \theta)$.

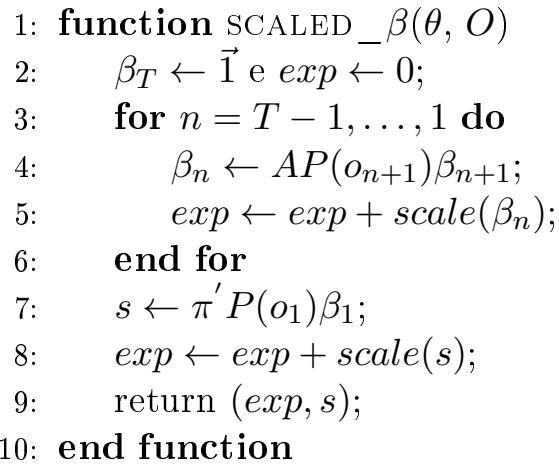

A função scale é dada por

Require: $\mathrm{O}$ vetor de números de ponto flutuante $v$.

Ensure: O inteiro mexp e o vetor $v^{*}$, do mesmo tipo e tamanho de $v$.

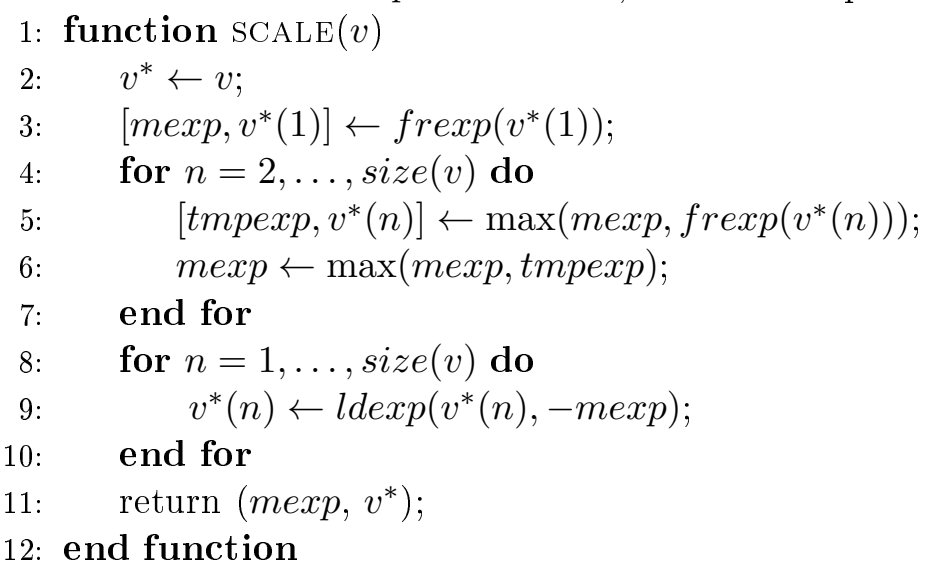

Comparamos nosso procedimento de normalização com o tradicional. O experimento foi realizado com o modelo de Markov oculto a seguir

$$
A=\left(\begin{array}{ll}
0.3067 & 0.6933 \\
0.6951 & 0.3049
\end{array}\right), B=\left(\begin{array}{ll}
0.7169 & 0.2831 \\
0.0665 & 0.9335
\end{array}\right) \text { e } \pi=\left(\begin{array}{c}
0.4 \\
0.6
\end{array}\right) .
$$


A tabela abaixo compara os resultados das duas abordagens. A coluna scaled traz os resultados obtidos com nossa versão da normalização. Na coluna log, apresentamos os resultados obtidos com o algoritmo $l o g \_\beta$. Nesse caso, a função $\log$ foi avaliado com a MPFR. Esse experimento foi realizado nas mesmas condições do anterior.

\begin{tabular}{|c|c|c|c|}
\hline T & scaled & log & $\mid$ log-scaled $\mid$ \\
\hline 10 & 0.00616949 & 0.00616949 & $7.80626 \mathrm{e}-18$ \\
\hline 20 & $3.06144 \mathrm{e}-06$ & $3.06144 \mathrm{e}-06$ & $9.74088 \mathrm{e}-21$ \\
\hline 50 & $2.49836 \mathrm{e}-15$ & $2.49836 \mathrm{e}-15$ & $2.32714 \mathrm{e}-29$ \\
\hline 100 & $3.70022 \mathrm{e}-30$ & $3.70022 \mathrm{e}-30$ & $6.30584 \mathrm{e}-45$ \\
\hline 150 & $6.93633 \mathrm{e}-42$ & $6.93633 \mathrm{e}-42$ & $4.11655 \mathrm{e}-55$ \\
\hline 200 & $3.56547 \mathrm{e}-55$ & $3.56547 \mathrm{e}-55$ & $2.14438 \mathrm{e}-68$ \\
\hline 250 & $2.72636 \mathrm{e}-72$ & $2.72636 \mathrm{e}-72$ & $3.93607 \mathrm{e}-85$ \\
\hline 300 & $3.31647 \mathrm{e}-85$ & $3.31647 \mathrm{e}-85$ & $2.97748 \mathrm{e}-98$ \\
\hline 400 & $3.44266 \mathrm{e}-116$ & $3.44266 \mathrm{e}-116$ & $1.02 \mathrm{e}-129$ \\
\hline
\end{tabular}

Tabela 4.1: Comparação entre as normalizações padrão e nossa alternativa.

A figura abaixo nos dá os tempos necessários para obter a tabela.

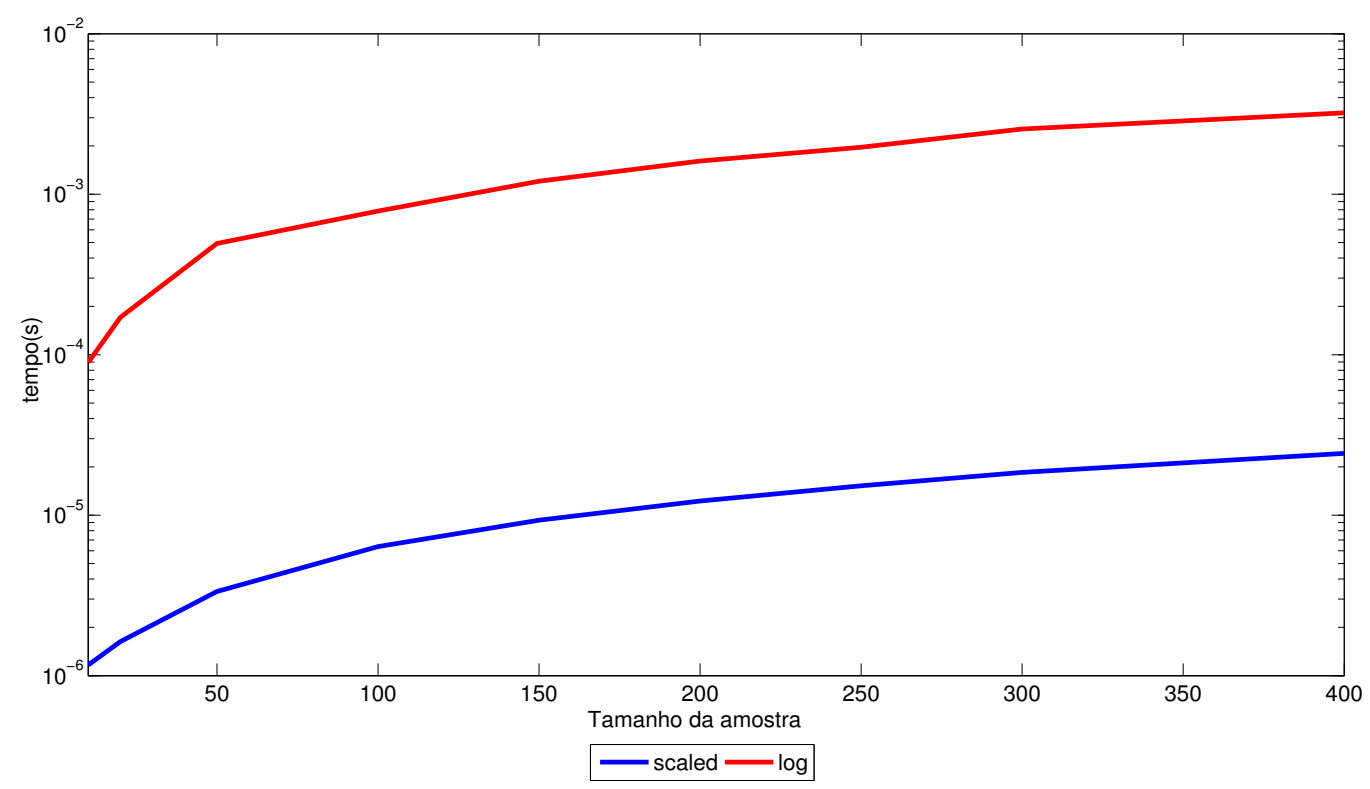

Figura 4.2: Tempo necessário para a avaliação de $\mathbb{P}(O \mid \theta)$ em amostras de tamanho $T$, dadas na tabela anterior. A linha azul se refere à nossa estratégia de normalização. A linha vermelha nos dá o tempo da normalização com log.

A tabela e o gráfico deixam claro que nosso algoritmo é compatível com o padrão em termos de precisão e cerca de 100 vezes mais eficiente.

\subsubsection{Função de Verossimilhança}

Nesta seção definimos a função de verossimilhança. Ela desempenha um papel fundamental no próximo capítulo onde buscamos maximizá-la. Dados o conjunto de observações $O$, temos

$$
L(\theta, O)=L_{O}(\theta)=\mathbb{P}(O \mid \theta)=\pi^{\prime} P\left(o_{1}\right) A \ldots A P\left(o_{T}\right) \overrightarrow{1} .
$$


A diferença entre $\mathbb{P}(O \mid \theta)$ e $L(\theta)$ é sutil. No primeiro caso, conhecemos o conjunto de observações e os parâmetros do modelo. No segundo, a probabilidade é dada como função do espaço paramétrico.

A função de verossimilhança nos dá a probabilidade de um modelo parametrizado por $\theta=$ $(A, B, \pi)$ gerar os dados observados. Se a tripla $\theta^{*}$ maximiza $L$, diremos que esse é um estimador de máxima verossimilhança. Determinar tais estimadores é o assunto central da tese, como veremos no capítulo 5. Por enquanto, nos restringimos a calcular $L$ e suas derivadas.

O cálculo de $L(\theta)$ é feito com a recursão backward, da mesma forma que $\mathbb{P}(O \mid \theta)$. Portanto, o algoritmo scaled_ $\beta$ apresentado acima resolve o problema. Mais ainda, essa recursão nos dá um caminho para avaliar $\nabla L(\theta)$ e $\nabla^{2} L(\theta)$. A partir da definição de $\beta$, temos

$$
\frac{\partial \beta_{n}}{\partial x}(\theta)=\frac{\partial A}{\partial x} P\left(o_{n+1}\right) \beta_{n+1}+A \frac{\partial P\left(o_{n+1}\right)}{\partial x} \beta_{n+1}+A P\left(o_{n+1}\right) \frac{\partial \beta_{n+1}}{\partial x} .
$$

Dessa forma,

$$
\begin{aligned}
& \frac{\partial \beta_{n}}{\partial a_{i j}}(\theta)=\chi_{i j} P\left(o_{n+1}\right) \beta_{n+1}+A P\left(o_{n+1}\right) \frac{\partial \beta_{n+1}}{\partial a_{i j}} \quad i, j=1, \ldots, N . \\
& \frac{\partial \beta_{n}}{\partial b_{i j}}(\theta)=A \frac{\partial P\left(o_{n+1}\right)}{\partial b_{i j}} \beta_{n+1}+A P\left(o_{n+1}\right) \frac{\partial \beta_{n+1}}{\partial b_{i j}} \quad i=1, \ldots, N \text { e } j=1, \ldots, M .
\end{aligned}
$$

Onde $\chi_{i j}$ é a matriz indicadora, com 1 na entrada $(i, j)$ e 0 nas demais. Lembrando que $P\left(o_{n}\right)=$ $\operatorname{diag}\left(b_{1 o_{n}}, \ldots, b_{N o_{n}}\right)$, temos

$$
\frac{\partial P\left(o_{n}\right)}{\partial b_{i j}}= \begin{cases}\chi_{i j} & \text { se } j=o_{n} \\ 0 & \text { caso contrário. }\end{cases}
$$

Além disso,

$$
\frac{\partial \beta_{n}}{\partial \pi_{i}}(\theta)=0 \quad i=1, \ldots, N \text { e } n=1, \ldots, T .
$$

Uma vez que calculamos as derivadas de $\beta$, podemos calcular $\nabla L$ a partir de

$$
\frac{\partial L}{\partial x}(\theta)=\frac{\partial \pi^{\prime}}{\partial x} P\left(o_{1}\right) \beta_{1}+\pi^{\prime} \frac{\partial P\left(o_{1}\right)}{\partial x} \beta_{1}+\pi^{\prime} P\left(o_{1}\right) \frac{\partial \beta_{1}}{\partial x} .
$$

Segue daí que,

$$
\begin{aligned}
\frac{\partial L}{\partial a_{i j}}(\theta) & =\pi^{\prime} P\left(o_{1}\right) \frac{\partial \beta_{1}}{\partial a_{i j}}, \\
\frac{\partial L}{\partial b_{i j}}(\theta) & =\pi^{\prime} \frac{\partial P\left(o_{1}\right)}{\partial b_{i j}} \beta_{1}+\pi^{\prime} P\left(O_{1}\right) \frac{\partial \beta_{1}}{\partial b_{i j}} .
\end{aligned}
$$

Além disso, como $\beta_{1}$ não depende de $\pi$

$$
\frac{\partial L}{\partial \pi}(\theta)=P\left(o_{1}\right) \beta_{1}
$$

Aplicando novamente as regras do cálculo, temos

$$
\begin{aligned}
\frac{\partial \beta_{n}}{\partial x \partial y}(\theta) & =\frac{\partial A}{\partial x \partial y} P\left(o_{n+1}\right) \beta_{n+1}+\frac{\partial A}{\partial x} \frac{\partial P\left(o_{n+1}\right)}{\partial y} \beta_{n+1}+\frac{\partial A}{\partial x} P\left(o_{n+1}\right) \frac{\partial \beta_{n+1}}{\partial y}+ \\
& +\frac{\partial A}{\partial y} \frac{\partial P\left(o_{n+1}\right)}{\partial x} \beta_{n+1}+A \frac{\partial P\left(o_{n+1}\right)}{\partial x \partial y} \beta_{n+1}+A \frac{\partial P\left(o_{n+1}\right)}{\partial x} \frac{\partial \beta_{n+1}}{\partial y}+ \\
& +\frac{\partial A}{\partial y} P\left(o_{n+1}\right) \frac{\partial \beta_{n+1}}{\partial x}+A \frac{\partial P\left(o_{n+1}\right)}{\partial y} \frac{\partial \beta_{n+1}}{\partial x}+A P\left(o_{n+1}\right) \frac{\partial \beta_{n+1}}{\partial x \partial y}
\end{aligned}
$$


Portanto,

$$
\begin{aligned}
\frac{\partial \beta_{n}}{\partial a_{i j} \partial a_{p k}}(\theta) & =\chi_{i j} P\left(o_{n+1}\right) \frac{\partial \beta_{n+1}}{\partial a_{p k}}+\chi_{p k} P\left(o_{n+1}\right) \frac{\partial \beta_{n+1}}{\partial a_{i j}}+A P\left(o_{n+1}\right) \frac{\partial \beta_{n+1}}{\partial a_{i j} \partial a_{p k}} \\
\frac{\partial \beta_{n}}{\partial a_{i j} \partial b_{p k}}(\theta) & =\chi_{i j} \frac{\partial P\left(o_{n+1}\right)}{\partial b_{p k}} \beta_{n+1}+\chi_{i j} P\left(o_{n+1}\right) \frac{\partial \beta_{n+1}}{\partial b_{p k}}+A P\left(o_{n+1}\right) \frac{\partial \beta_{n+1}}{\partial a_{i j} \partial b_{p k}} \\
\frac{\partial \beta_{n}}{\partial b_{i j} \partial b_{p k}}(\theta) & =A \frac{\partial P\left(o_{n+1}\right)}{\partial b_{i j} \partial b_{p k}} \beta_{n+1}+A \frac{\partial P\left(o_{n+1}\right)}{\partial b_{i j}} \frac{\partial \beta_{n+1}}{\partial b_{p k}}+A \frac{\partial P\left(o_{n+1}\right)}{\partial b_{p k}} \frac{\partial \beta_{n+1}}{\partial b_{i j}}+ \\
& +A P\left(o_{n+1}\right) \frac{\partial \beta_{n+1}}{\partial b_{i j} \partial b_{p k}} \\
\frac{\partial \beta_{n}}{\partial \pi_{i} \partial x}(\theta) & =0 .
\end{aligned}
$$

Derivando duas vezes o termo geral de $L$, temos

$$
\begin{aligned}
\frac{\partial L}{\partial x \partial y}(\theta) & =\frac{\partial \pi^{\prime}}{\partial x \partial y} P\left(o_{1}\right) \beta_{1}+\frac{\partial \pi^{\prime}}{\partial x} \frac{\partial P\left(o_{1}\right)}{\partial y} \beta_{1}+\frac{\partial \pi^{\prime}}{\partial x} P\left(o_{1}\right) \frac{\partial \beta_{1}}{\partial y}+ \\
& +\frac{\partial \pi^{\prime}}{\partial y} \frac{\partial P\left(o_{1}\right)}{\partial x} \beta_{1}+\pi^{\prime} \frac{\partial P\left(o_{1}\right)}{\partial x \partial y} \beta_{1}+\pi^{\prime} \frac{\partial P\left(o_{1}\right)}{\partial x} \frac{\partial \beta_{1}}{\partial y}+ \\
& +\frac{\partial \pi^{\prime}}{\partial y} P\left(o_{1}\right) \frac{\partial \beta_{1}}{\partial x}+\pi^{\prime} \frac{\partial P\left(o_{1}\right)}{\partial y} \frac{\partial \beta_{1}}{\partial x}+\pi^{\prime} P\left(o_{1}\right) \frac{\partial \beta_{1}}{\partial x \partial y} .
\end{aligned}
$$

Finalmente,

$$
\begin{aligned}
\frac{\partial L}{\partial a_{i j} \partial a_{p k}}(\theta) & =\pi^{\prime} P\left(o_{1}\right) \frac{\partial \beta_{1}}{\partial a_{i j} \partial a_{p k}}, \\
\frac{\partial L}{\partial a_{i j} \partial b_{p k}}(\theta) & =\pi^{\prime} \frac{\partial P\left(o_{1}\right)}{\partial b_{p k}} \frac{\partial \beta_{1}}{\partial a_{i j}}+\pi^{\prime} P\left(o_{1}\right) \frac{\partial \beta_{1}}{\partial a_{i j} \partial b_{p k}}, \\
\frac{\partial L}{\partial b_{i j} \partial b_{p k}}(\theta) & =\pi^{\prime} \frac{\partial P\left(o_{1}\right)}{\partial b_{i j} \partial b_{p k}} \beta_{1}+\pi^{\prime} \frac{\partial P\left(o_{1}\right)}{\partial b_{i j}} \frac{\partial \beta_{1}}{\partial b_{p k}}+\pi^{\prime} \frac{\partial P\left(o_{1}\right)}{\partial b_{p k}} \frac{\partial \beta_{1}}{\partial b_{i j}}+\pi^{\prime} P\left(o_{1}\right) \frac{\partial \beta_{1}}{\partial b_{i j} \partial b_{p k}} \\
\frac{\partial L}{\partial \pi_{i} \partial x}(\theta) & =e_{i}^{\prime} \frac{\partial P\left(o_{1}\right)}{\partial x} \beta_{1}+e_{i}^{\prime} P\left(o_{1}\right) \frac{\partial \beta_{1}}{\partial x}
\end{aligned}
$$

Nesse caso, $e_{i}$ é o $i$-ésimo vetor canônico.

\subsection{Extensão Natural}

Mostramos agora que o cálculo das probabilidades intervalares através da extensão natural é lento. Isto acontece por causa do número elevado de trocas no modo de arredondamento. Sugerimos uma mudança na implementação que resolve esse problema. Comparamos a eficiência das duas abordagens.

O teorema fundamental da aritmética intervalar garante que podemos calcular $\mathbb{P}(O \mid \boldsymbol{\theta})$ a partir da equação 4.3. Para tanto, basta substituir as operações reais por intervalares. Dessa forma, temos

$$
L(\theta)=\pi^{\prime} A P\left(o_{1}\right) A P\left(o_{2}\right) \ldots A P\left(o_{T}\right) \overrightarrow{1}
$$

É claro que a extensão também se aplica à recursão backward. Ela nos dá

$$
\begin{aligned}
\boldsymbol{\beta}_{T} & =\overrightarrow{\mathbf{1}} \\
\boldsymbol{\beta}_{\boldsymbol{n}} & =\boldsymbol{A P}\left(o_{n+1}\right) \boldsymbol{\beta}_{n+1} \quad n=T-1, \ldots, 1 \\
\boldsymbol{L}(\boldsymbol{\theta}) & =\boldsymbol{\pi}^{\prime} \boldsymbol{P}\left(o_{1}\right) \boldsymbol{\beta}_{\mathbf{1}} .
\end{aligned}
$$


A recursão é simples e podemos implementá-la com qualquer biblioteca intervalar. No entanto, ela é bastante custosa. Segundo a definição $(2.11)$, são necessárias $8\left((T-1) N^{2}+N(T+1)\right)$ operações de ponto flutuante e $2\left((T-1) N^{2}+N(T+1)\right)$ trocas no modo de arredondamento para avaliar $\boldsymbol{L}(\boldsymbol{\theta})$ rigorosamente.

Observe agora que as entradas de $\boldsymbol{A}, \boldsymbol{\pi}$ e $\boldsymbol{P}\left(O_{n}\right)$ são probabilidades. Assim, os intervalos $\boldsymbol{a}_{\boldsymbol{i} \boldsymbol{j}}$, $\boldsymbol{b}_{\boldsymbol{i} \boldsymbol{j}}$ e $\boldsymbol{\pi}_{\boldsymbol{i}}$ são não negativos. Dessa forma, o produto

$$
\begin{aligned}
\boldsymbol{x} * \boldsymbol{y}= & {[\nabla(\min \{\inf (\boldsymbol{x}) \inf (\boldsymbol{y}), \inf (\boldsymbol{x}) \sup (\boldsymbol{y}), \sup (\boldsymbol{x}) \inf (\boldsymbol{y}), \sup (\boldsymbol{x}) \sup (\boldsymbol{y})\}),} \\
& \triangle(\max \{\inf (\boldsymbol{x}) \inf (\boldsymbol{y}), \inf (\boldsymbol{x}) \sup (\boldsymbol{y}), \sup (\boldsymbol{x}) \inf (\boldsymbol{y}), \sup (\boldsymbol{x}) \sup (\boldsymbol{y})\})]
\end{aligned}
$$

se reduz a

$$
\boldsymbol{x} * \boldsymbol{y}=[\nabla(\inf (\boldsymbol{x}) \inf (\boldsymbol{y})), \triangle(\sup (\boldsymbol{x}) \sup (\boldsymbol{y}))]
$$

pois não há produtos da forma $\inf (\boldsymbol{x}) \sup (\boldsymbol{y})$ ou $\inf (\boldsymbol{y}) \sup (\boldsymbol{x})$.

Como a soma intervalar é dada por $\boldsymbol{x}+\boldsymbol{y}=[\nabla(\inf (\boldsymbol{x})+\inf (\boldsymbol{y})), \Delta(\sup (\boldsymbol{x})+\sup (\boldsymbol{y}))]$, dividimos a recursão backward intervalar em duas partes, dadas por

$$
\begin{aligned}
\inf \left(\boldsymbol{\beta}_{\boldsymbol{n}}\right) & =\inf (\boldsymbol{A}) \inf \left(\boldsymbol{P}\left(o_{n+1}\right)\right) \inf \left(\boldsymbol{\beta}_{n+1}\right), \\
\sup \left(\boldsymbol{\beta}_{\boldsymbol{n}}\right) & =\sup (\boldsymbol{A}) \sup \left(\boldsymbol{P}\left(o_{n+1}\right)\right) \sup \left(\boldsymbol{\beta}_{n+1}\right) .
\end{aligned}
$$

Além disso,

$$
\begin{aligned}
\inf (\boldsymbol{L}(\boldsymbol{\theta})) & =\inf \left(\boldsymbol{\pi}^{\prime}\right) \inf \left(\boldsymbol{P}\left(o_{1}\right)\right) \inf \left(\boldsymbol{\beta}_{\mathbf{1}}\right) \\
\sup (\boldsymbol{L}(\boldsymbol{\theta})) & =\sup \left(\boldsymbol{\pi}^{\prime}\right) \sup \left(\boldsymbol{P}\left(o_{1}\right)\right) \sup \left(\boldsymbol{\beta}_{\mathbf{1}}\right) .
\end{aligned}
$$

Portanto, reduzimos o número de trocas no modo de arredondamento a dois, independente do tamanho da amostra ou do número de parâmetros. Mais ainda, a quantidade de operações em ponto flutuante para uma avaliação intervalar, é duas vezes o número de operações em modelos reais.

Para garantir o rigor, ajustamos o modo de arredondamento para menos infinito $(\nabla)$ e realizamos todas operações de $\inf (\boldsymbol{L}(\boldsymbol{\theta}))$. Em seguida mudamos o modo de arredondamento para mais infinito $(\triangle)$ e realizamos o cálculo de $\sup (\boldsymbol{L}(\boldsymbol{\theta}))$. A normalização proposta na seção anterior é válida, desde que realizada no vetor $\sup (\boldsymbol{L}(\boldsymbol{\theta}))$. Em seguida, o expoente resultante também é aplicado a $\inf (\boldsymbol{L}(\boldsymbol{\theta}))$. O algoritmo a seguir avalia $\boldsymbol{L}(\boldsymbol{\theta})$ normalizado.

Require: Os parâmetros intervalares, $\boldsymbol{\theta}$ e o conjunto de observações $O$.

Ensure: O inteiro exp e o intervalo $\boldsymbol{L}$, tais que $\boldsymbol{L} * 2^{\exp }=\boldsymbol{L}(\boldsymbol{\theta})$.

1: function SCALED_ $\boldsymbol{\beta}(\boldsymbol{\theta}, O)$

2: $\quad \beta_{T} \leftarrow \overrightarrow{1}, \exp \leftarrow 0$, exp_lower $\leftarrow 0$;

3: $\quad$ round_mode $\leftarrow \nabla()$;

4: $\quad$ for $n=T-1, \ldots, 1$ do

$$
\begin{aligned}
& \beta_{n} \leftarrow \inf (\boldsymbol{A}) \inf \left(\boldsymbol{P}\left(\boldsymbol{o}_{\boldsymbol{n}+\mathbf{1}}\right)\right) \beta_{n+1} ; \\
& \text { exp_lower } \leftarrow \text { exp_lower }+\operatorname{scale}\left(\beta_{n}\right) ;
\end{aligned}
$$

\section{end for}

$s_{-}$lower $\leftarrow \inf \left(\boldsymbol{\pi}^{\prime}\right) \inf \left(\boldsymbol{P}\left(\boldsymbol{o}_{\mathbf{1}}\right)\right) \beta_{1}$;

exp_lower $\leftarrow$ exp_lower + scale $\left(s \_l o w e r\right)$;

$\beta_{T} \leftarrow \overrightarrow{1} ;$

round_mode $\leftarrow \triangle()$;

for $n=T-1, \ldots, 1$ do

$$
\beta_{n} \leftarrow \sup (\boldsymbol{A}) \sup \left(\boldsymbol{P}\left(\boldsymbol{o}_{\boldsymbol{n}+\mathbf{1}}\right)\right) \beta_{n+1} ;
$$$$
\exp \leftarrow \exp +\operatorname{scale}\left(\beta_{n}\right) ;
$$

\section{end for}

16: $\quad s_{\text {_upper }} \leftarrow \sup \left(\boldsymbol{\pi}^{\prime}\right) \sup \left(\boldsymbol{P}\left(\boldsymbol{o}_{\mathbf{1}}\right)\right) \beta_{1}$; 
17: $\quad \exp \leftarrow$ exp + scale $\left(s \_u p p e r\right)$;

18: $\quad s \_l$ lower $\leftarrow s$ _lower $* 2^{\text {exp_lower-exp }}$;

19: $\quad \boldsymbol{L} \leftarrow\left[s_{-}\right.$lower, $s_{-}$upper $]$;

20: $\quad$ return $(\exp , \boldsymbol{L})$;

\section{1: end function}

A função scale é a mesma da seção anterior. O algoritmo calcula os extremos em dois laços separados. Na linha 18 normalizamos o limitante inferior do intervalo com o expoente obtido no cálculo do limitante superior. Esse passo é necessário para garantir que os dois extremos tem expoente comum.

O algoritmo abaixo calcula $\boldsymbol{L}(\boldsymbol{\theta})$ e $\nabla \boldsymbol{L}(\boldsymbol{\theta})$, ambos normalizados. Ele adota a seguinte notação

$$
d \beta_{n}(x)=\left(\begin{array}{c}
\frac{\partial}{\partial x} \beta_{n}(1) \\
\vdots \\
\frac{\partial}{\partial x} \beta_{n}(N)
\end{array}\right) .
$$

Aqui, $\beta_{n}$ é o vetor da recursão backward na $n$-ésima iteração.

Require: Os parâmetros intervalares, $\boldsymbol{\theta}$ e o conjunto de observações $O$.

Ensure: O inteiro exp, o intervalo $\boldsymbol{L}$ e a caixa $\boldsymbol{d} \boldsymbol{L}$, tais que $\boldsymbol{L} * 2^{e x p}=\boldsymbol{L}(\boldsymbol{\theta})$ e $\boldsymbol{d} \boldsymbol{L} * 2^{\exp }=\nabla \boldsymbol{L}(\boldsymbol{\theta})$.

1: function SCALED_ $\boldsymbol{\nabla} \boldsymbol{\beta}(\boldsymbol{\theta}, O)$

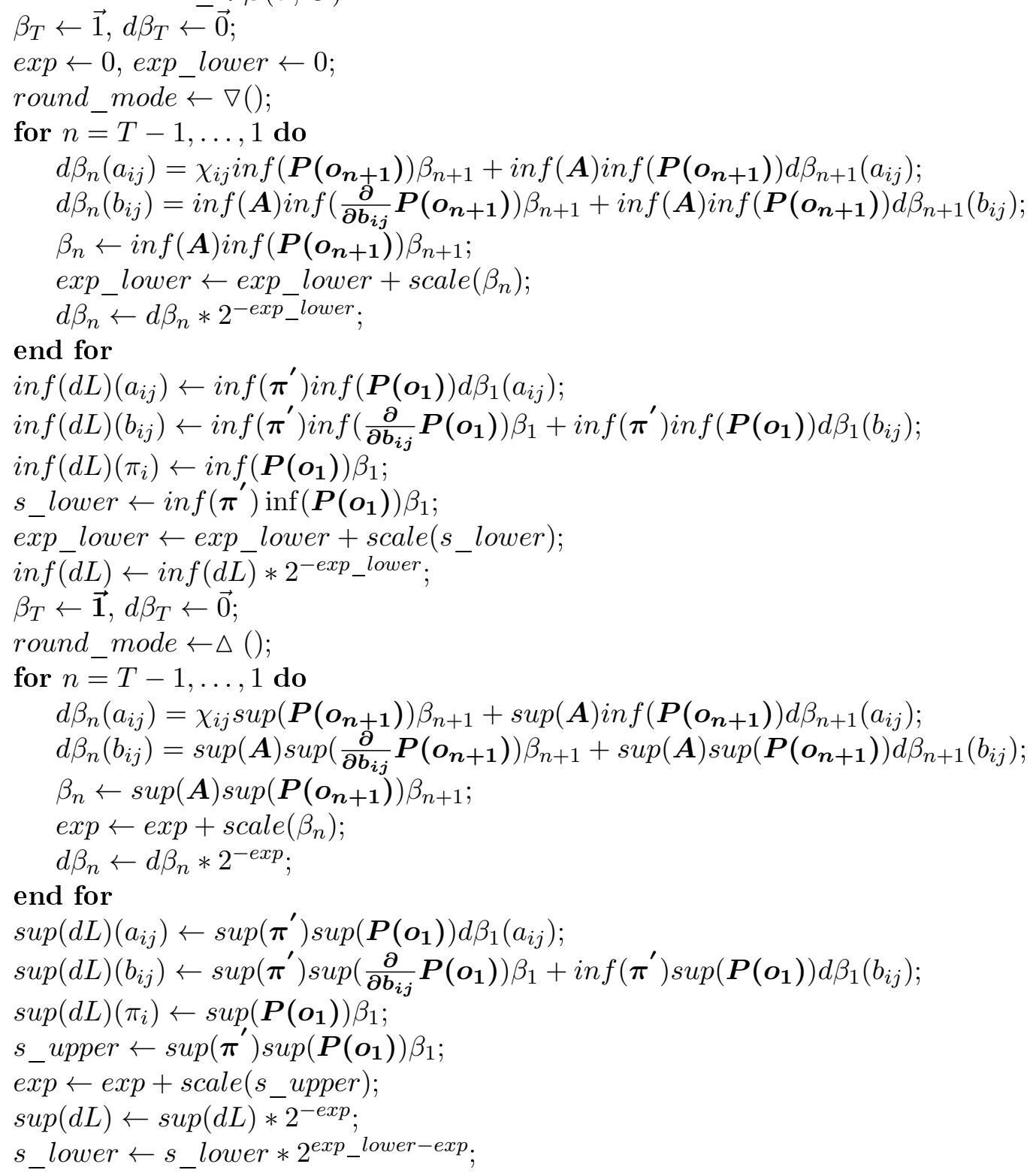




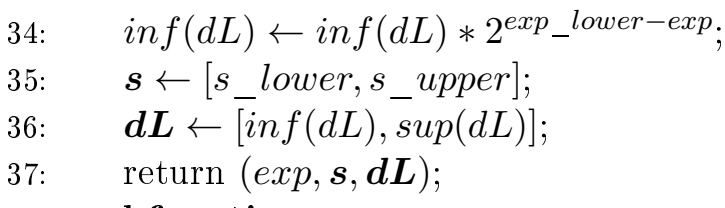

\section{8: end function}

O cálculo da Hessiana também é realizado com 2 troca no modo de arredondamento. Ele segue a mesma ideia do algoritmo acima, incorporando as equações (4.4) - (4.5).

\subsubsection{Experimentos numéricos}

Concluímos a seção comparando a extensão natural padrão com nossa modificação. O teste foi realizado com modelos ocultos de dimensões diferentes. Ele leva em conta apenas o tempo de execução dos algoritmos. Em cada caso, partimos de um modelo real, parametrizado por $\theta=$ $(A, B, \pi)$ e construímos $\boldsymbol{\theta}$, com centro $\theta$ e raio 0.1 . Os parâmetros do modelo intervalar são da forma

$$
\begin{aligned}
\boldsymbol{a}_{\boldsymbol{i j}} & =\left[a_{i j}-0.1, a_{i j}+0.1\right] \cap[0,1], \\
\boldsymbol{b}_{\boldsymbol{i j}} & =\left[b_{i j}-0.1, b_{i j}+0.1\right] \cap[0,1], \\
\boldsymbol{\pi}_{\boldsymbol{i}} & =\left[\pi_{i}-0.1, \pi_{i}+0.1\right] \cap[0,1] .
\end{aligned}
$$

As figuras abaixo nos dão o tempo, em segundos, da avaliação de $\boldsymbol{L}(\boldsymbol{\theta}), \nabla \boldsymbol{L}(\boldsymbol{\theta})$ e $\nabla^{2} \boldsymbol{L}(\boldsymbol{\theta})$. Os experimentos foram realizados em uma máquina com processador core-i $i$, compilador $i c c++14.0 .2$ e opções de compilação -O3, -frounding-math e -NDEBUG. Quando nos referirmos ao $M M_{2,2}$ intervalar, ele foi construído a partir de $\theta=(A, B, \pi)$ onde

$$
A=\left(\begin{array}{ll}
0.604069 & 0.395931 \\
0.380030 & 0.619970
\end{array}\right), B=\left(\begin{array}{ll}
0.173443 & 0.826557 \\
0.669679 & 0.330321
\end{array}\right) \text { e } \pi=\left(\begin{array}{l}
1 \\
0
\end{array}\right) .
$$

O $M M_{2,4}$ intervalar tem centro

$$
A=\left(\begin{array}{ll}
0.604069 & 0.395931 \\
0.380030 & 0.619970
\end{array}\right), B=\left(\begin{array}{llll}
0.056246 & 0.268044 & 0.452509 & 0.223201 \\
0.255112 & 0.364469 & 0.257975 & 0.122445
\end{array}\right) \text { e } \pi=(1) .
$$

O centro do $M M O_{3,3}$ intervalar é

$$
A=\left(\begin{array}{lll}
0.427486 & 0.280191 & 0.292323 \\
0.448874 & 0.290034 & 0.261093 \\
0.404480 & 0.193006 & 0.402514
\end{array}\right), B=\left(\begin{array}{lll}
0.072407 & 0.345063 & 0.582530 \\
0.174239 & 0.340006 & 0.485754 \\
0.331051 & 0.157129 & 0.511820
\end{array}\right) \text { e } \pi=\left(\begin{array}{l}
1 \\
0 \\
0
\end{array}\right)
$$

Finalmente, o $\mathrm{MMO}_{3,5}$ é centrado em

$$
A=\left(\begin{array}{lll}
0.427486 & 0.280191 & 0.292323 \\
0.448874 & 0.290034 & 0.261093 \\
0.404480 & 0.193006 & 0.402514
\end{array}\right), \pi=\left(\begin{array}{l}
1 \\
0 \\
0
\end{array}\right)
$$

e

$$
B=\left(\begin{array}{lllll}
0.039181 & 0.186719 & 0.315217 & 0.155481 & 0.303402 \\
0.260034 & 0.184054 & 0.087359 & 0.284557 & 0.183996 \\
0.285657 & 0.052686 & 0.029911 & 0.112301 & 0.519446
\end{array}\right)
$$

Os outros modelos são grandes demais para descrevermos aqui. 

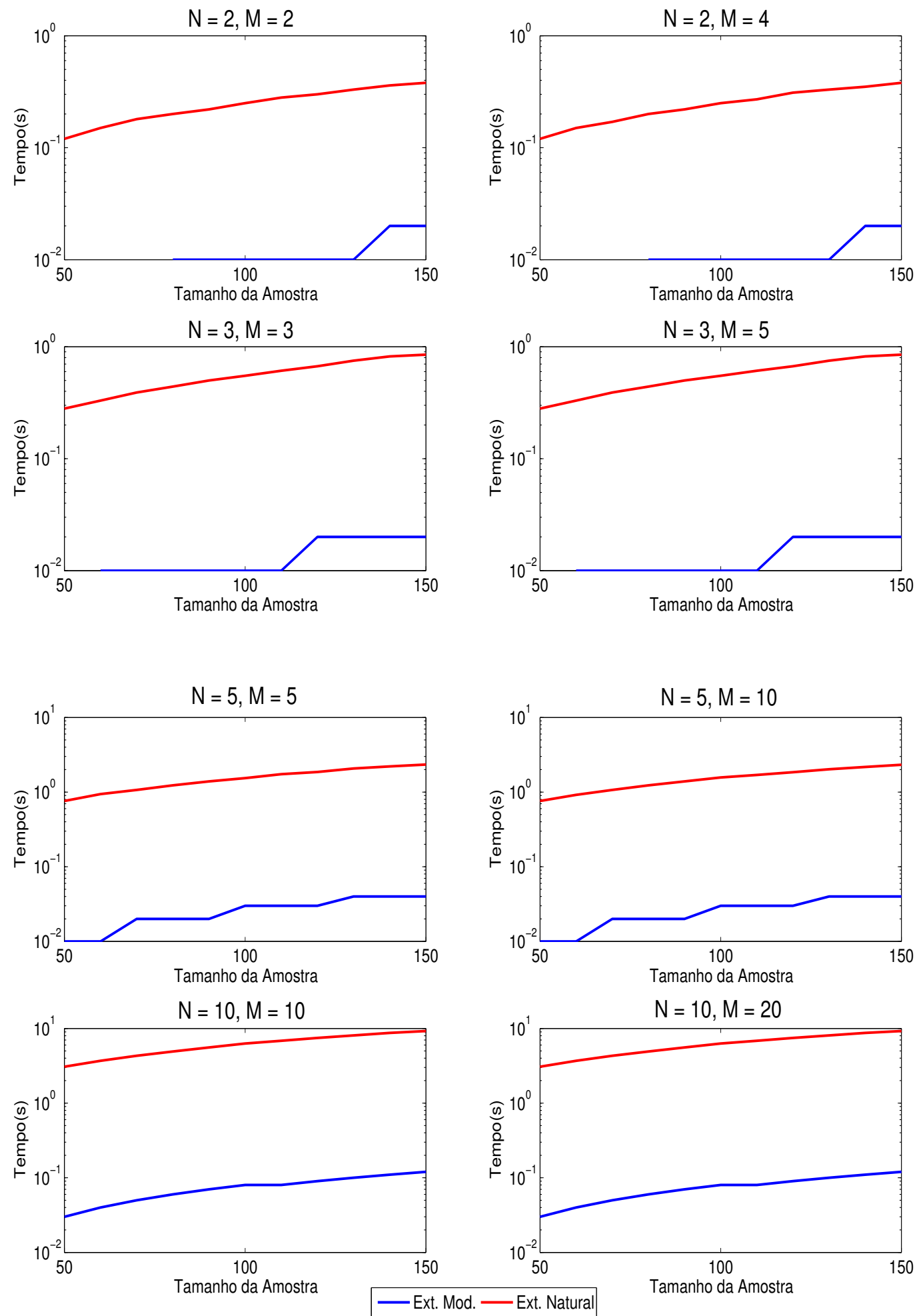

Figura 4.3: Comparação entre nossa modificação da extensão natural e a forma padrão no cálculo de $\boldsymbol{L}(\boldsymbol{\theta})$. 

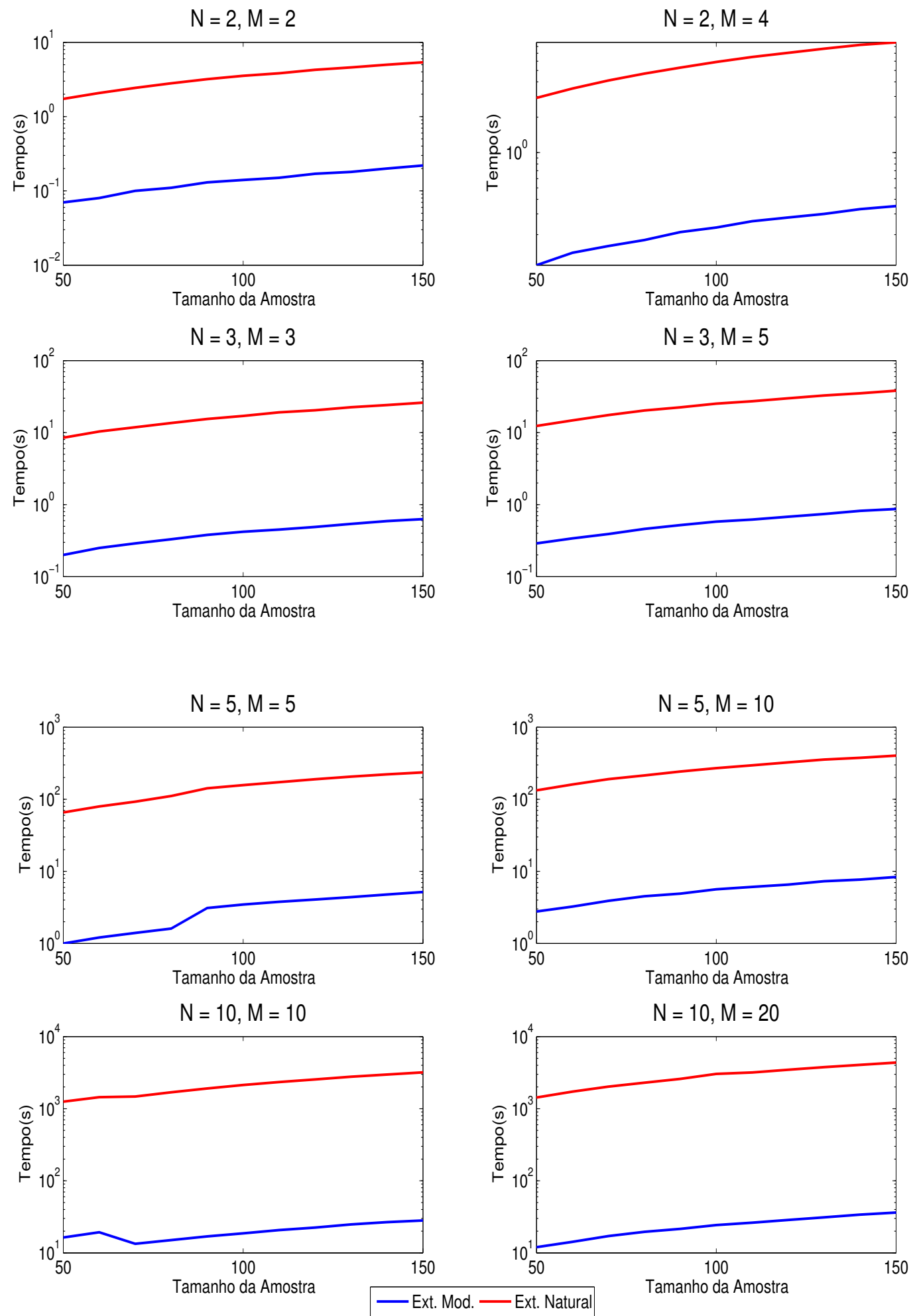

Figura 4.4: Comparação entre nossa modificação da extensão natural e a forma padrão no cálculo de $\nabla \boldsymbol{L}(\boldsymbol{\theta})$. 

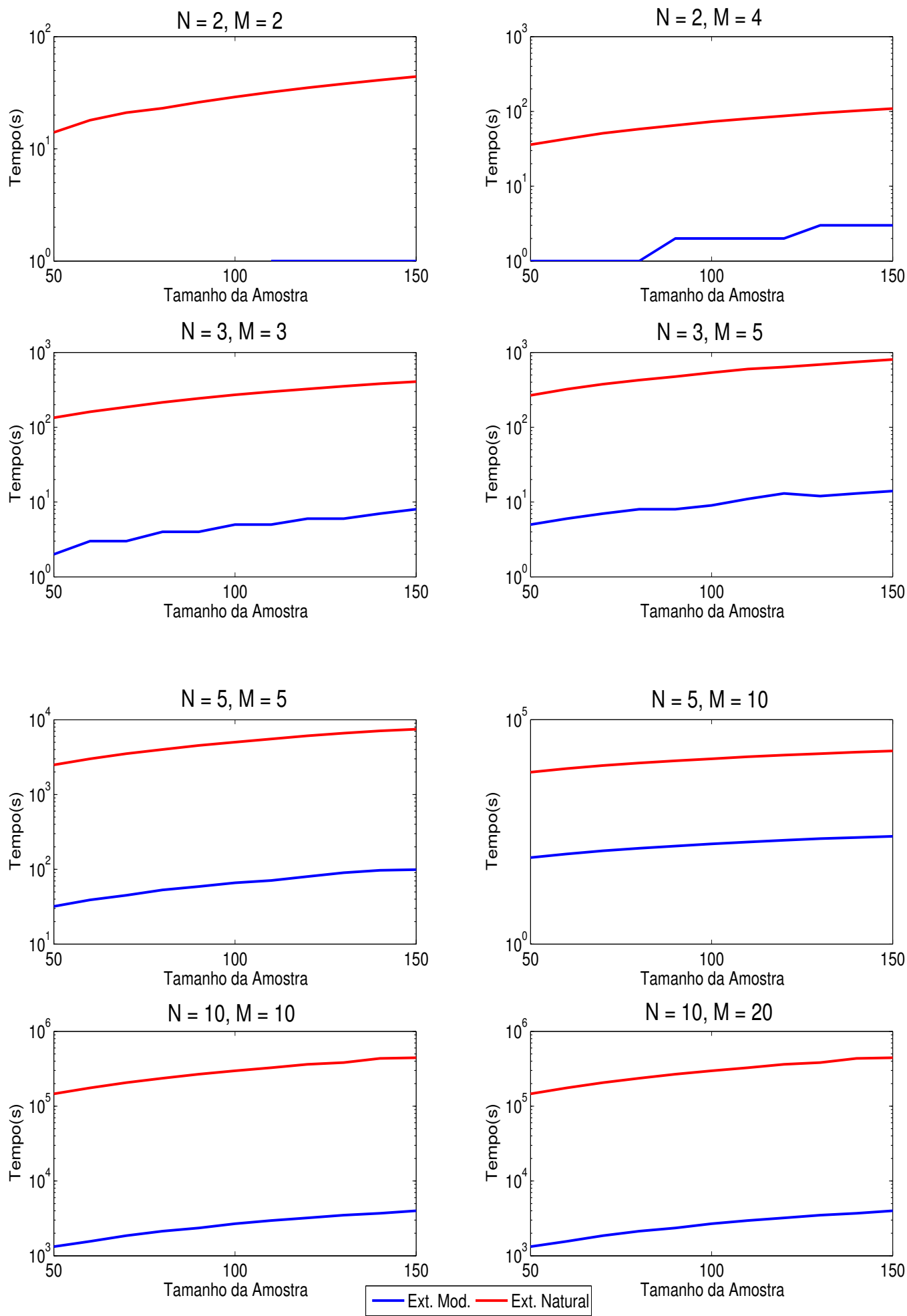

Figura 4.5: Comparação entre nossa modificação da extensão natural e a forma padrão no cálculo de $\nabla^{2} \boldsymbol{L}(\boldsymbol{\theta})$. 
As figuras mostram ganhos significativos de nossa modificação da extensão natural. A vantagem aumenta conforme variamos o tamanho da amostra ou o número de parâmetros do modelo. Para $\boldsymbol{L}(\boldsymbol{\theta})$, nossa abordagem é cerca de 16 vezes mais eficiente para o $M M O_{2,2}$ e chega a 250 vezes para o $M M O_{10,20}$.

Nossa modificação da extensão natural diminui o número de trocas no modo de arredondamento. Isso traz ganhos significativos no desempenho do cálculo, mas não melhora os limitantes obtidos. Por exemplo, considere o modelo de Markov oculto intervalar parametrizado por

$$
\boldsymbol{A}=\left(\begin{array}{ll}
{[0.25,0.75]} & {[0.25,0.75]} \\
{[0.25,0.75]} & {[0.25,0.75]}
\end{array}\right), \boldsymbol{\pi}=\left(\begin{array}{l}
{[0.25,0.75]} \\
{[0.25,0.75]}
\end{array}\right)
$$

e

$$
\boldsymbol{B}=\left(\begin{array}{lll}
{[0.25,0.75]} & {[0.25,0.75]} & {[0.25,0.75]} \\
{[0.25,0.75]} & {[0.25,0.75]} & {[0.25,0.75]}
\end{array}\right)
$$

O conjunto $O=\{1,3\}$ tem probabilidade $\mathbb{P}(O \mid \boldsymbol{\theta})=[0.015,1.265]$. Nesse caso, embora o cálculo seja rigoroso, a informação do limitante superior é inútil. Na próxima seção apresentamos uma nova extensão intervalar, que é tão eficiente quanto a proposta aqui e que produz intervalos mais estreitos.

\subsection{Extensão com Programação Linear}

Na seção anterior modificamos a extensão natural para diminuir o número de trocas do modo de arredondamento. Apresentamos agora uma extensão intervalar baseada em programação linear. Ela é tão eficiente quanto nossa modificação da extensão natural, mas produz intervalos mais estreitos. Nosso ponto de partida é o termo geral da recursão backward, dado por

$$
\beta_{n}=A P\left(o_{n+1}\right) \beta_{n+1} .
$$

Lembrando que $P\left(o_{n+1}\right)$ é uma matriz diagonal, o produto $P\left(o_{n+1}\right) \beta_{n+1}$ se escreve

$$
c=\left(\begin{array}{c}
p_{1}\left(o_{n+1}\right) \beta_{1} \\
\vdots \\
p_{N}\left(o_{n+1}\right) \beta_{N}
\end{array}\right) .
$$

Dessa forma, o termo geral da recursão intervalar é $\boldsymbol{\beta}_{\boldsymbol{n}}=\boldsymbol{A} \boldsymbol{c}$. Ao realizar esse produto com a extensão natural, consideramos matrizes que pertencem a $\boldsymbol{A}$ mas que não são de transição. Por exemplo, se

$$
\boldsymbol{A}=\left(\begin{array}{cc}
{\left[\frac{1}{3}, \frac{2}{3}\right]} & {\left[\frac{1}{4}, \frac{3}{4}\right]} \\
\frac{4}{5} & \frac{1}{5}
\end{array}\right) \text { e } \boldsymbol{c}=\left(\begin{array}{c}
0.55 \\
0.45
\end{array}\right)
$$

então a matriz real

$$
A=\left(\begin{array}{cc}
\frac{2}{3} & \frac{3}{4} \\
\frac{4}{5} & \frac{1}{5}
\end{array}\right)
$$

pertence a $\boldsymbol{A}$, mas não é de transição. No entanto, o vetor

$$
v=\left(\begin{array}{cc}
\frac{2}{3} & \frac{3}{4} \\
\frac{4}{5} & \frac{1}{5}
\end{array}\right)\left(\begin{array}{l}
0.55 \\
0.45
\end{array}\right)=\left(\begin{array}{l}
0.7042 \\
0.5300
\end{array}\right)
$$

pertence a $\boldsymbol{A} \boldsymbol{c}$. A inclusão desses vetores no conjunto $\boldsymbol{A} \boldsymbol{c}$ leva à sobrestimação de $\mathbb{P}(O \mid \boldsymbol{\theta})$.

Uma forma de lidar com esse problema, é incorporar a restrição $a_{11}+a_{12}=1$ ao cálculo. Dessa forma, se $\boldsymbol{\beta}_{\boldsymbol{n}+\mathbf{1}}$ (1) é a primeira entrada de $\boldsymbol{\beta}_{\boldsymbol{n}+\mathbf{1}}$ então um limitante superior rigoroso de 
$\sup \left(\boldsymbol{\beta}_{\boldsymbol{n}}(\mathbf{1})\right)$ é

$$
\begin{array}{ll}
\max & 0.55 a_{11}+0.45 a_{12} \\
\text { S.t } & a_{11}+a_{12}=1 \\
& a_{11} \in\left[\frac{1}{3}, \frac{2}{3}\right] \\
& a_{12} \in\left[\frac{1}{4}, \frac{3}{4}\right] .
\end{array}
$$

Da mesma forma $\inf \left(\boldsymbol{\beta}_{\boldsymbol{n}}(\mathbf{1})\right)$ está limitado inferiormente por

$$
\begin{array}{ll}
\min & 0.55 a_{11}+0.45 a_{12} \\
\text { S.t } & a_{11}+a_{12}=1 \\
& a_{11} \in\left[\frac{1}{3}, \frac{2}{3}\right] \\
& a_{12} \in\left[\frac{1}{4}, \frac{3}{4}\right] .
\end{array}
$$

Em geral o vetor $\boldsymbol{c}$ não é degenerado. Nesses casos, novamente observamos que tanto $\beta_{n+1}$ quanto $P\left(O_{n+1}\right)$ são probabilidades. Sendo assim, a caixa $\boldsymbol{c}$ é não negativa e a simplificação da multiplicação continua válida. Generalizando a ideia acima temos $\boldsymbol{\beta}_{\boldsymbol{n}}(i)=[\beta, \bar{\beta}]$ onde

$$
\begin{aligned}
& \underline{\beta}=\min \inf \left(\boldsymbol{c}_{\mathbf{1}}\right) a_{i 1}+\ldots+\inf \left(\boldsymbol{c}_{\boldsymbol{N}}\right) a_{i N} \\
& \text { S.t } \quad a_{i 1}+\ldots+a_{i N}=1 \\
& \quad a_{i j} \in \boldsymbol{a}_{\boldsymbol{i j}}
\end{aligned}
$$

e

$$
\begin{aligned}
& \bar{\beta}=\max \sup \left(\boldsymbol{c}_{\mathbf{1}}\right) a_{i 1}+\ldots+\sup \left(\boldsymbol{c}_{\boldsymbol{N}}\right) a_{i N} \\
& \text { S.t } \quad a_{i 1}+\ldots+a_{i N}=1 \\
& a_{i j} \in \boldsymbol{a}_{\boldsymbol{i j}}
\end{aligned}
$$

Finalmente, se $\boldsymbol{c}=\boldsymbol{P}\left(\boldsymbol{o}_{\mathbf{1}}\right) \boldsymbol{\beta}_{\mathbf{1}}$ então aplicamos a mesma ideia à última etapa do cálculo para obter $\mathbb{P}(O \mid \theta)=[\underline{p}, \bar{p}]$, onde

$$
\begin{aligned}
& \underline{p}=\min \inf \left(\boldsymbol{c}_{\mathbf{1}}\right) \pi_{1}+\ldots+\inf \left(\boldsymbol{c}_{\boldsymbol{N}}\right) \pi_{N} \\
& \text { S.t } \quad \pi_{1}+\ldots+\pi_{N}=1 \\
& \quad \pi_{i} \in \boldsymbol{\pi}_{\boldsymbol{i}}
\end{aligned}
$$

e

$$
\begin{aligned}
& \bar{p}=\max \sup \left(\boldsymbol{c}_{\mathbf{1}}\right) \pi_{1}+\ldots+\sup \left(\boldsymbol{c}_{\boldsymbol{N}}\right) \pi_{N} \\
& \text { S.t } \quad \pi_{1}+\ldots+\pi_{N}=1 \\
& \quad \pi_{i} \in \boldsymbol{\pi}_{\boldsymbol{i}} .
\end{aligned}
$$

Os problemas de programação linear propostos tem solução se o modelo intervalar for consistente. Para distinguir a extensão com programação linear da natural, escrevemos $\boldsymbol{L}_{P L}(\boldsymbol{\theta})$ e $\boldsymbol{L}_{N T}(\boldsymbol{\theta})$. Quando não houver ambigüidade, escrevemos apenas $\boldsymbol{L}(\boldsymbol{\theta})$ para a extensão com programação linear. A partir da construção que fizemos, segue o resultado abaixo.

Proposição 3 Seja $\boldsymbol{\theta}=(\boldsymbol{A}, \boldsymbol{P}, \boldsymbol{\pi})$ um modelo de Markov oculto consistente. Se $\theta=(A, P, \pi)$ é um modelo real que satisfaz $(A, \pi) \in(\boldsymbol{A}, \boldsymbol{\pi})$ então

$$
\inf \left(\boldsymbol{L}_{P L}(\boldsymbol{\theta})\right) \leq L(\theta) \leq \sup \left(\boldsymbol{L}_{P L}(\boldsymbol{\theta})\right) .
$$


Além disso,

$$
\boldsymbol{L}_{P L}(\boldsymbol{\theta}) \subseteq \boldsymbol{L}_{N T}(\boldsymbol{\theta}) .
$$

A proposição afirma que $\boldsymbol{L}_{P L}(\boldsymbol{\theta})$ contém a variação $\overline{\boldsymbol{L}_{O}(\boldsymbol{\theta})}$, restrita a modelos consistentes. Ela também nos diz que nossa abordagem produz limitantes, superiores ou inferiores, pelo menos tão bons quanto os da extensão natural.

Podemos obter limitantes mais estreitos para as derivadas de $\boldsymbol{L}(\boldsymbol{\theta})$ a partir da mesma ideia. Lembrando que o termo geral das derivadas de $\beta$ é

$$
\frac{\partial \boldsymbol{\beta}_{\boldsymbol{n}}}{\partial x}=\frac{\partial \boldsymbol{P}\left(\boldsymbol{o}_{\boldsymbol{n}+\mathbf{1}}\right)}{\partial x} \boldsymbol{A} \boldsymbol{\beta}_{\boldsymbol{n}+\mathbf{1}}+\boldsymbol{A} \frac{\partial \boldsymbol{P}\left(\boldsymbol{o}_{\boldsymbol{n}+\mathbf{1}}\right)}{\partial x} \boldsymbol{\beta}_{\boldsymbol{n}+\mathbf{1}}+\boldsymbol{A P}\left(\boldsymbol{o}_{\boldsymbol{n}+\mathbf{1}}\right) \frac{\partial \boldsymbol{\beta}_{\boldsymbol{n}+\mathbf{1}}}{\partial x},
$$

basta aplicar nossa extensão aos produtos $\boldsymbol{A} \frac{\partial \boldsymbol{P}\left(\boldsymbol{o}_{\boldsymbol{n}+1}\right)}{\partial x} \boldsymbol{\beta}_{\boldsymbol{n}+\mathbf{1}}$ e $\boldsymbol{A} \boldsymbol{P}\left(\boldsymbol{o}_{\boldsymbol{n}+\boldsymbol{1}}\right) \frac{\partial \boldsymbol{\beta}_{\boldsymbol{n}+\boldsymbol{1}}}{\partial x}$. O mesmo pode ser feito com os termos da Hessiana.

A extensão com programação linear é tão eficiente quanto a modificação do caso natural. Todas as quantidades envolvidas no cálculo da probabilidade e de suas derivadas são não negativas. Dessa forma, podemos resolver cada problema de programação linear sem trocas no modo de arredondamento. Além disso, cada um desses problemas é simples o suficiente para ser resolvido diretamente, sem aplicarmos o algoritmo simplex.

\subsubsection{Experimentos Numéricos}

Concluímos o capítulo repetindo o experimento da seção anterior. Nosso interesse é avaliar a qualidade dos limitantes obtidos com cada método. O modelos centrais nesta seção são os mesmos do experimento anterior. Por outro lado, o raio $r$ é fundamental nesse teste. Como o cálculo a partir das extensões é rigoroso, $\left\|w\left(\boldsymbol{L}_{P L}(\boldsymbol{\theta})\right)-w\left(\boldsymbol{L}_{N T}(\boldsymbol{\theta})\right)\right\| \rightarrow 0$ conforme $r \rightarrow 0$. Os raios testados nesse experimento são $r \in\{0.001,0.01,0.1\}$.

Observamos que em modelos intervalos cujos parâmetros têm raio moderado, nossa extensão intervalar produz limitantes superiores mais estreitos do que a natural. Como esperado, essa vantagem diminui conforme raio do modelo. 

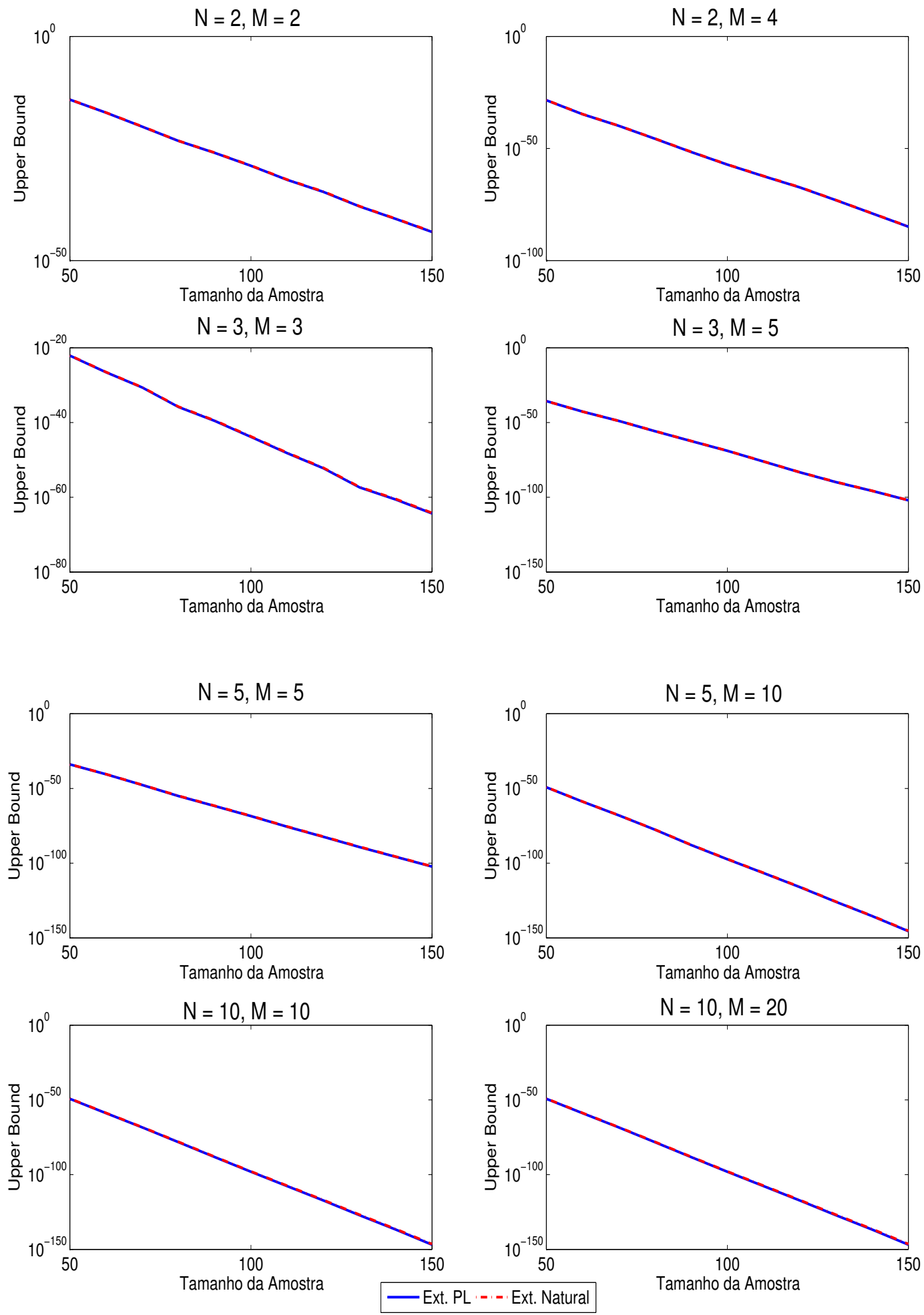

Figura 4.6: Comparação entre os limitantes superiores de $\boldsymbol{L}_{P L}(\boldsymbol{\theta})$ e $\boldsymbol{L}_{N T}(\boldsymbol{\theta})$. Os parâmetros dos modelos têm raio 0.001 

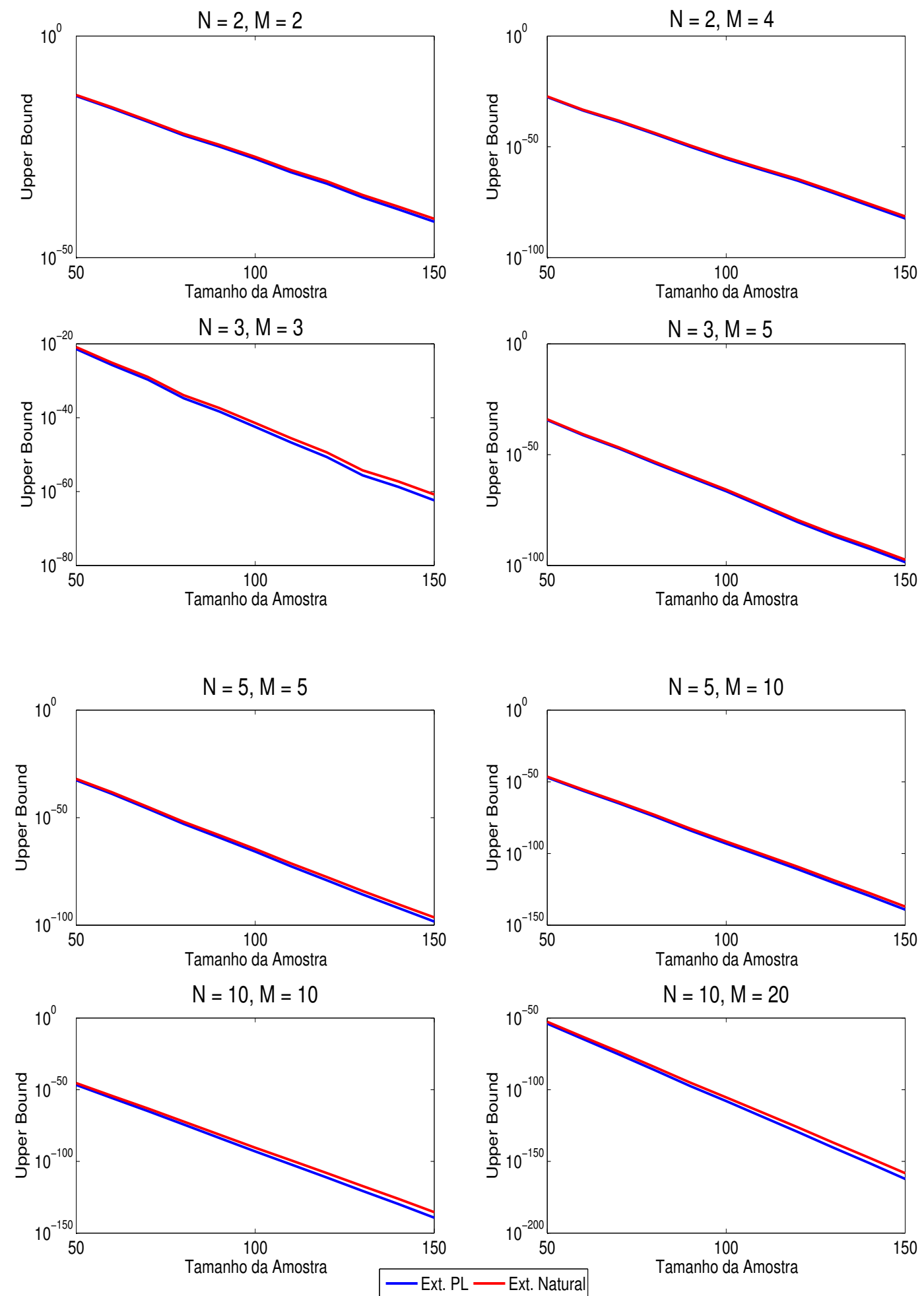

Figura 4.7: Comparação entre os limitantes superiores de $\boldsymbol{L}_{P L}(\boldsymbol{\theta})$ e $\boldsymbol{L}_{N T}(\boldsymbol{\theta})$. Os parâmetros dos modelos têm raio 0.01 

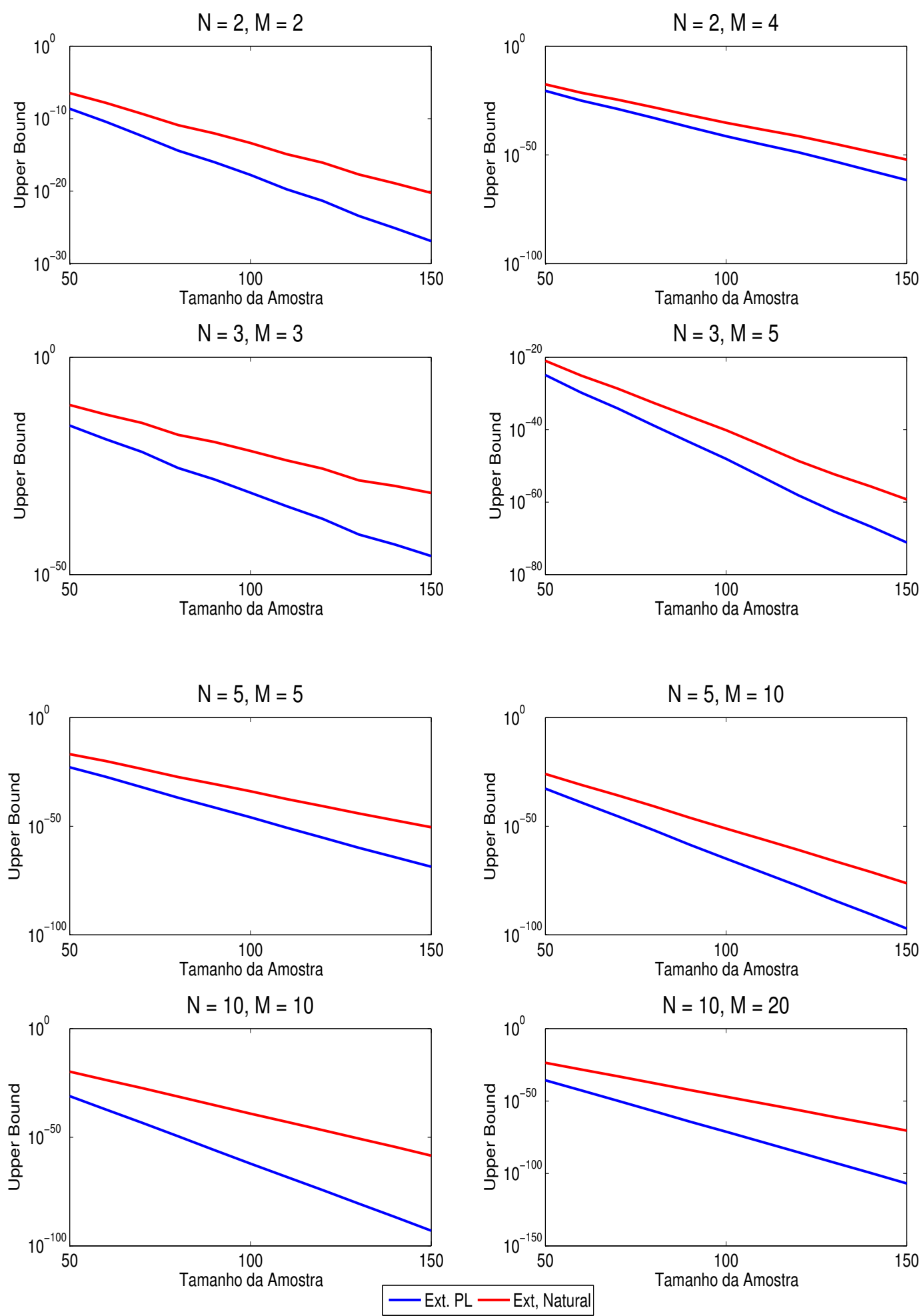

Figura 4.8: Comparação entre os limitantes superiores de $\boldsymbol{L}_{P L}(\boldsymbol{\theta})$ e $\boldsymbol{L}_{N T}(\boldsymbol{\theta})$. Os parâmetros dos modelos têm raio 0.1 


\section{Capítulo 5}

\section{Estimando Parâmetros}

Este capítulo estuda a estimação de parâmetros em modelos de Markov ocultos. Dado o conjunto de observações $O=\left(o_{1}, \ldots, o_{T}\right)$, o número de estados ocultos $N$ e de símbolos $M$, o problema no qual estamos interessados é

$$
\begin{array}{rlr}
\max z & =\pi^{\prime} P\left(o_{1}\right) A \ldots A P\left(o_{T}\right) \overrightarrow{1} \\
\sum_{j=1}^{N} a_{i j} & =1 \quad \forall i=1, \ldots, N . \\
\sum_{j=1}^{M} b_{i j} & =1 \quad \forall i=1, \ldots, N . \\
\sum_{i=1}^{N} \pi_{i} & =1 & \\
a_{i j}, b_{i j}, \pi_{i} & \geq 0 .
\end{array}
$$

Assim como nos capítulos anteriores, o modelo de Markov oculto é parametrizado por $\theta=(A, B, \pi)$. O par $(A, \pi)$ é uma cadeia de Markov com $N$ estados. A matriz $B$ representa as probabilidades de emissão de uma mistura discreta no conjunto $\{1, \ldots, M\}$. Além disso,

$$
P\left(o_{n}\right):=\left(\begin{array}{cccc}
b_{1 o_{n}} & 0 & \ldots & 0 \\
0 & b_{2 o_{n}} & \ldots & 0 \\
\vdots & \vdots & \vdots & \vdots \\
0 & 0 & \ldots & b_{N o_{n}}
\end{array}\right) .
$$

Resolver (5.1)-(5.5) significa determinar todos os parâmetros que maximizam a função objetivo globalmente. Para tanto, implementamos o interval branch and bound, descrito em Kearfott (1996) e Hansen e Walster (2004). Esse é um algoritmo geral de otimização global. Ele explora sistematicamente caixas que contém o conjunto viável, eliminando regiões que comprovadamente não têm soluções.

O interval branch and bound é um algoritmo custoso. Sua implementação ingênua é incapaz de estimar os parâmetros de um MMO em tempo hábil. Nosso objetivo é apresentar procedimentos que acelerem sua convergência, considerando a estrutura do problema. As principais contribuições do capítulo são:

1. Comparação de métodos locais, para obter melhores limitantes inferiores do máximo global.

2. Novos testes para redução e exclusão de caixas, baseados no sistema KKT e nas simetrias do problema.

3. Análise de procedimentos de aceleração da convergência, comuns em aritmética intervalar. 
4. Comparação da nossa implementação com o resolvedor de otimização global BARON.

O capítulo está dividido da seguinte forma. Na seção 5.1 estudamos as condições KKT de primeira ordem do problema (5.1) - (5.5). A seção 5.2 apresenta os métodos de otimização local que implementamos. Realizamos experimentos numéricos com os métodos locais na seção 5.3. Apresentamos o interval branch and bound na seção 5.4. Em 5.5 discutimos as simetrias do problema. Na seção 5.6, estudamos procedimentos de aceleração da convergência. A comparação com o BARON é feita na seção 5.7 .

\subsection{Condições KKT}

Apresentamos condições necessárias de primeira ordem para que $\theta^{*}$ seja solução de (5.1) - (5.5). A principal referência desta seção é o capítulo 12 de Nocedal e Wright (2006). Considere o problema geral de otimização

$$
\begin{array}{rlr}
\max z & =f(x) \\
c_{i}(x) & =0 \quad i=1, \ldots, p . \\
h_{i}(x) & \geq 0 \quad i=p+1, \ldots, p+m .
\end{array}
$$

onde $f: \mathbb{R}^{n} \rightarrow \mathbb{R}, c_{i}: \mathbb{R}^{n} \rightarrow \mathbb{R}$ e $h_{i}: \mathbb{R}^{n} \rightarrow \mathbb{R}$ são funções diferenciáveis. Associado a ele, temos a função Lagrangiana $\mathcal{L}: \mathbb{R}^{n+p+m} \rightarrow \mathbb{R}$, dada por

$$
\mathcal{L}(x, \lambda, \mu):=f(x)-\sum_{i=1}^{p} \lambda_{i} c_{i}(x)-\sum_{i=p+1}^{p+m} \mu_{i} h_{i}(x)
$$

Definição 13 (Restrições Ativas) Dados

$$
\Omega:=\left\{x \in \mathbb{R}^{n} \mid c_{1}(x)=0, \ldots, c_{p}(x)=0 \text { e } h_{p+1}(x) \geq 0 \ldots h_{p+q}(x) \geq 0\right\}
$$

e $x \in \Omega$, o conjunto $\mathcal{A}(x):=\{1, \ldots, p\} \cup\left\{i \mid h_{i}(x)=0\right\}$ é chamado de restrições ativas em $x$.

Definição 14 (LICQ) Dados $\Omega, x \in \Omega$ e $\mathcal{A}(x)$ diremos que $x$ satisfaz LICQ se o conjunto de vetores $\left\{\nabla c_{i}(x), \nabla h_{i}(x) \mid i \in \mathcal{A}(x)\right\}$ é linearmente independente.

O teorema abaixo dá condições necessárias de primeira ordem para que $x^{*}$ seja solução de (5.6). Ele está demonstrado em Nocedal e Wright (2006).

Teorema 5 (Condições Necessárias de Primeira Ordem) Se $x^{*}$ é solução de 5.6 que satisfaz $L I C Q$ então existem vetores $\lambda^{*} \in \mathbb{R}^{p}$ e $\mu^{*} \in \mathbb{R}^{m}$ tais que

$$
\begin{array}{rll}
\nabla_{x} \mathcal{L}\left(x^{*}, \lambda^{*}, \mu *\right) & =0 & \\
c_{i}(x) & =0 & i=1, \ldots, p, \\
h_{i}(x) & \geq 0 & i=p+1, \ldots, m, \\
\mu_{i} & \geq 0 & i=p+1, \ldots, m, \\
\mu_{i} h_{i}\left(x^{*}\right) & =0 & i=p+1, \ldots, m .
\end{array}
$$

Os vetores $\lambda^{*}$ e $\mu *$ são chamados multiplicadores de Lagrange.

Observe que o teorema não diz nada sobre a natureza da solução. O ponto $x^{*}$ pode ser um máximo local ou global. O resultado é ainda mais fraco. Pontos de mínimo e selas também satisfazem as condições KKT. Sendo assim, todo algoritmo baseado em (5.7)-(5.11) deve adotar testes adicionais para excluir soluções indesejadas.

Para usar o teorema na estimação de parâmetros em modelos de Markov ocultos, precisamos provar o seguinte resultado: 
Proposição 4 Se $\theta=(A, B, \pi)$ satisfaz (5.2) - (5.5) então ele também satisfaz LICQ.

Demonstração - Considere as restrições (5.2) e (5.5) com $i=1$. Elas tem a forma

$$
\begin{array}{rlrl}
a_{11}+a_{12}+\ldots+a_{1 N} & =1 \\
a_{11} & \geq 0 \\
& & & \\
& & a_{1 N} & \geq 0 .
\end{array}
$$

Se $\theta$ é viável então ao menos um $a_{1 j}$ deve ser não nulo. Seja $D_{1}$ a matriz cujas colunas são os gradientes das restrições ativas de (5.13). Dessa forma,

$$
D_{1}=\left(\begin{array}{ccccc}
1 & 1 & 0 & \ldots & 0 \\
1 & 0 & 1 & \ldots & 0 \\
1 & \vdots & \vdots & \ldots & 0 \\
1 & 0 & 0 & \ldots & 1
\end{array}\right)
$$

Como no máximo $N-1$ das desigualdades pertencem à $\mathcal{A}(\theta)$, as colunas de $D_{1}$ formam um conjunto linearmente independente. Aplicando este raciocínio às demais restrições, temos que matriz dos vetores gradientes associados à $\mathcal{A}(\theta)$ tem a forma

$$
\left(\begin{array}{cccc}
D_{1} & 0 & \ldots & 0 \\
0 & D_{2} & \ldots & 0 \\
\vdots & \vdots & \vdots & 0 \\
0 & 0 & \ldots & D_{2 N+1}
\end{array}\right) .
$$

Nesse caso, $D_{1}, \ldots D_{N}$ são matrizes associadas às restrições (5.2) e (5.5), $D_{N+1}, \ldots, D_{2 N}$ se referem à (5.3), (5.5) e $D_{2 N+1}$ está associada às restrições (5.4) e (5.5). É claro que as colunas dessa matriz formam um conjunto linearmente independente.

Uma vez que as hipóteses do teorema estão satisfeitas, para que um modelo $\theta^{*}$ seja solução de (5.1) - (5.5), devem existir $\lambda^{*} \in \mathbb{R}^{2 N+1}$ e $\mu^{*} \in \mathbb{R}^{N^{2}+N M+N}$ tais que

$$
\begin{array}{rlrl}
\frac{\partial L}{\partial a_{i j}}\left(\theta^{*}\right)-\lambda_{A_{i}}^{*}-\mu_{a_{i j}}^{*} & =0 & & \forall i, j=1, \ldots, N . \\
\frac{\partial L}{\partial b_{i j}}\left(\theta^{*}\right)-\lambda_{B_{i}}^{*}-\mu_{b_{i j}}^{*}=0 & & \forall i=1, \ldots, N \text { e } j=1, \ldots, M . \\
\frac{\partial L}{\partial \pi_{i}}\left(\theta^{*}\right)-\lambda_{\pi}^{*}-\mu_{\pi_{i}}^{*}=0 & & \forall i, j=1, \ldots, N . \\
\sum_{j=1}^{N} a_{i j} & =1 & & \forall i=1, \ldots, N . \\
\sum_{j=1}^{M} b_{i j} & =1 & & \\
\sum_{i=1}^{N} \pi_{i} & =1 & & \\
\mu_{a_{i j}}^{*} a_{i j}^{*} & =0 & & \forall i, j=1, \ldots, N . \\
\mu_{b_{i j}}^{*} b_{i j}^{*} & =0 & \forall i=1, \ldots, N \text { e } j=1, \ldots, M . \\
\mu_{\pi_{i}}^{*} \pi_{i}^{*} & =0 & \forall i, j=1, \ldots, N . \\
\mu_{a_{i j}}^{*}, \mu_{b_{i j}}^{*} \mu_{\pi_{i}}^{*} \geq 0 . & &
\end{array}
$$


Para facilitar a notação, indexamos os elementos de $\lambda^{*}$ e $\mu^{*}$ com as respectivas restrições. O cálculo de $\frac{\partial L}{\partial x}\left(\theta^{*}\right)$ é realizado com recursão backward, que apresentamos no capítulo anterior.

A partir das equações (5.14) - (5.22) é fácil obter limitantes para os multiplicadores de Lagrange. Considere novamente as restrições (5.13). As equações KKT associadas a elas são

$$
\begin{aligned}
\frac{\partial L}{\partial a_{11}}\left(\theta^{*}\right)-\lambda_{A_{1}}^{*}-\mu_{a_{11}}^{*} & =0 \\
\frac{\partial L}{\partial a_{12}}\left(\theta^{*}\right)-\lambda_{A_{1}}^{*}-\mu_{a_{12}}^{*} & =0 \\
\vdots & \\
\frac{\partial L}{\partial a_{1 N}}\left(\theta^{*}\right)-\lambda_{A_{1}}^{*}-\mu_{a_{1 N}}^{*} & =0 \\
\sum_{i=1}^{N} A_{1} & =1 \\
\mu_{a_{1 j}}^{*} a_{1 j}^{*} & =0 \\
\mu_{a_{1 j}}^{*} & \geq 0 .
\end{aligned}
$$

Aqui, $A_{i}$ é a $i$-ésima linha de $A$. As equações (5.26) e (5.27) nos dizem que existe pelo menos um índice $k$ tal que $\mu_{a_{1 k}}=0$. Considerando a $k$-ésima equação de (5.23)-(5.25) temos

$$
\frac{\partial L}{\partial a_{1 k}}\left(\theta^{*}\right)=\lambda_{A_{1}}^{*}
$$

Dessa forma, se $\boldsymbol{\theta}$ é a caixa onde procuramos pelos máximos globais de (5.1)-(5.5) então

$$
\min _{j=1 \ldots N} \inf \left(\frac{\partial \boldsymbol{L}}{\partial \boldsymbol{a}_{1 j}}(\boldsymbol{\theta})\right) \leq \lambda_{A_{1}}^{*}
$$

e

$$
\lambda_{A_{1}}^{*} \leq \max _{j=1 \ldots N} \sup \left(\frac{\partial \boldsymbol{L}}{\partial \boldsymbol{a}_{\mathbf{1 j}}}(\boldsymbol{\theta})\right) .
$$

Finalmente, a desigualdade (5.28) nos diz que $\mu_{a_{1 j}} \in\left[0, \overline{\mu_{a_{1 j}}}\right]=\boldsymbol{\mu}_{\boldsymbol{a}_{1 j}}$. Como conhecemos limitantes da caixa $\boldsymbol{\lambda}_{\boldsymbol{A}_{\mathbf{1}}}^{*}$, temos

$$
\frac{\partial L}{\partial a_{1 j}}(\theta)-\lambda_{A_{1}}^{*}=\mu_{a_{1 j}}
$$

Subtraindo os intervalos à esquerda da equação segue que $\overline{\mu_{a_{i j}}}=\sup \left(\frac{\boldsymbol{\partial} \boldsymbol{L}}{\boldsymbol{\partial \boldsymbol { a } _ { \mathbf { 1 j } }}}(\boldsymbol{\theta})\right)-\inf \left(\boldsymbol{\lambda}_{\boldsymbol{A}_{\mathbf{1}}}^{*}\right)$.

Mostramos que para limitar os multiplicadores de Lagrange, basta conhecer as derivadas da caixa inicial $\boldsymbol{\theta}$. Uma maneira de resolver (5.1)-(5.5), é limitar as variáveis do sistema (5.14)-(5.22) e, em seguida, buscar todos os zeros desse sistema. Ao longo do processo, excluímos as raízes que não podem ser máximos globais. Essa é uma abordagem simples, que podemos implementar com o INTSOLVER ou o GLOBSOL Kearfott (1996). Por outro lado, ela aumenta o número de variáveis de decisão. Além disso, estamos interessados apenas nos parâmetros $\theta$ e não em seus multiplicadores de Lagrange. Por essas razões não seguimos esse caminho.

\subsection{Estimação Local dos Parâmetros}

Algoritmos locais desempenham um papel importante no interval branch and bound. Em um problema de maximização, eles dão limitantes inferiores do máximo global. Quanto melhor for o limitante, mais rapidamente descartamos caixas que não contém máximos globais. Em geral, isso leva a um número menor de iterações do método global. Por outro lado, resolvemos muitos problemas 
locais no processo de busca. Um algoritmo local lento, torna o método global inviável.

Nesta seção, apresentamos duas alternativas locais que implementamos para resolver (5.1) (5.5). Os algoritmos escolhidos foram o Baum-Welch, descrito em Rabiner (1990), e o método dos gradientes projetados, presente em Birgin et al. (2000).

\subsubsection{Baum-Welch}

O método de Baum-Welch é o precursor do algoritmo EM, descrito em Dempster et al. (1977). Ele foi introduzido por Baum et al. (1970). Inicialmente, o método contemplava apenas misturas discretas. Desde então, ele foi generalizado. Atualmente é o método mais usado para estimar parâmetros em modelos de Markov ocultos.

O algoritmo de Baum-Welch é iterativo. Segundo Rabiner (1990), o iterando é da forma

$$
\begin{aligned}
\pi_{i}^{n+1} & =\frac{\pi_{i}^{n} \frac{\partial L}{\partial \pi_{i}}\left(\theta^{n}\right)}{\sum_{k=1}^{N} \pi_{k}^{n} \frac{\partial L}{\partial \pi_{k}}\left(\theta^{n}\right)}, \\
a_{i j}^{n+1} & =\frac{a_{i j}^{n} \frac{\partial L}{\partial a_{i j}}\left(\theta^{n}\right)}{\sum_{k=1}^{N} a_{i k}^{n} \frac{\partial L}{\partial a_{i k}}\left(\theta^{n}\right)}, \\
b_{i j}^{n+1} & =\frac{b_{i j}^{n} \frac{\partial L}{\partial b_{i j}}\left(\theta^{n}\right)}{\sum_{k=1}^{M} b_{i k}^{n} \frac{\partial L}{\partial b_{i k}}\left(\theta^{n}\right)} .
\end{aligned}
$$

Aqui, $\pi_{i}^{n+1}$ é a $i$-ésima coordenada do vetor $\pi$ na iteração $n+1$. A partir dessas equações, é claro que

$$
\begin{aligned}
& \sum_{k=1}^{N} \pi_{k}^{n+1}=1 \\
& \sum_{k=1}^{N} a_{i k}^{n+1}=1, \quad i=1, \ldots, N \\
& \sum_{k=1}^{M} b_{i k}^{n+1}=1 . \quad i=1, \ldots, N
\end{aligned}
$$

Ou seja, se $\theta^{0}$ é viável então as demais aproximações $\theta^{n}$ serão viáveis. Além disso, Rabiner prova que $L\left(\theta^{n+1}\right) \geq L\left(\theta^{n}\right)$, para todo $n \geq 0$.

Dado um modelo $\theta^{0}$ viável, o algoritmo de Baum-Welch termina quando algum critério de parada é satisfeito. Em nossa implementação, concluímos o algoritmo quando a condição

$$
L\left(\theta^{n+1}\right)-L\left(\theta^{n}\right)<\epsilon * L\left(\theta^{n+1}\right)
$$

é satisfeita para um $\epsilon>0$ conveniente. Como o algoritmo sempre gera pontos viáveis, é fácil interrompê-lo se um número máximo de iterações for atingido. O pseudo-código de nossa implementação é dado abaixo.

Require: $O=\left(o_{1}, \ldots, o_{T}\right), \theta^{0}$ viável, $N>0, M>0, \epsilon>0$ e max_iter $>0$.

Ensure: $\theta^{*}$ viável e tal que $L_{O}\left(\theta^{*}\right) \geq L_{O}\left(\theta^{0}\right)$.

1: function $\operatorname{BaumWeLch}\left(O, \theta^{0}, N, M, \epsilon\right.$, max_iter $)$

2: $\quad$ iter $\leftarrow 0$;

3: $\quad$ while true do

4: $\quad \pi_{i}^{1} \leftarrow$ expressão da equação (5.29); 
5: $\quad a_{i j}^{1} \leftarrow$ expressão da equação $(5.30)$

6: $\quad b_{i j}^{1} \leftarrow$ expressão da equação $(5.31)$

7: $\quad$ iter $\leftarrow$ iter +1 ;

8: $\quad$ if $L_{O}\left(\theta^{1}\right)-L_{O}\left(\theta^{0}\right)>\epsilon L_{O}\left(\theta^{0}\right) \|$ iter $\geq$ max_iter then

9: $\quad$ break;

10: $\quad$ end if

11: $\quad \theta^{0} \leftarrow \theta^{1}$;

12: end while

13: $\quad$ return $\theta^{1}$;

14: end function

Nesse algoritmo $L_{O}(\theta)=L(\theta)$. Escrevemos $L_{O}(\theta)$ para destacar que $L$ depende das observações $O=\left(o_{1}, \ldots, o_{T}\right)$.

\subsubsection{Gradientes Projetados Espectrais - SPG}

O método dos gradientes projetados é descrito em Calamai e Moré (1987). Ele resolve problemas da forma

$$
\begin{aligned}
\min z & =f(x) \\
x & \in \Omega .
\end{aligned}
$$

Aqui, $f: \mathbb{R}^{n} \rightarrow \mathbb{R}$ é uma função diferenciável e $\Omega$ um conjunto convexo. Esse método também é iterativo e sua iteração e dada por

$$
x_{n+1}:=\operatorname{Pr}\left(x_{n}-\alpha_{n} \nabla f\left(x_{n}\right)\right) .
$$

A função $\operatorname{Pr}(x)$ é a projeção de $x$ em $\Omega$. Ela é dada por,

$$
\operatorname{Pr}(x):=\operatorname{argmin}\{\|z-x\| \mid z \in \Omega\} .
$$

O escalar $\alpha_{n}>0$ é o passo da busca linear. Nocedal e Wright (2006) apresenta diversas formas de escolher $\alpha_{n}$ de modo a garantir a convergência do método. $\mathrm{O}$ algoritmo de gradientes projetados produz pontos viáveis a cada iteração, independente de $\theta^{0}$ ser ou não viável. Birgin et al. (2000) sugere que o algoritmo termine com sucesso, devolvendo $x^{*}$ como resposta se $\left\|\operatorname{Pr}\left(\nabla f\left(x^{*}\right)\right)-x^{*}\right\|<\epsilon$, $\operatorname{com} \epsilon>0$.

Calcular $\operatorname{Pr}(x)$ é a parte mais custosa do método dos gradientes projetados. Em geral, o algoritmo tem valor prático somente se pudermos resolver (5.33) com eficiência. Vamos interpretar o problema da projeção da seguinte forma. Dados um MMO intervalar e consistente, que é parametrizado pela caixa $\boldsymbol{\theta}=(\boldsymbol{A}, \boldsymbol{B}, \boldsymbol{\pi})$ e um ponto $x \notin \boldsymbol{\theta}$ então $\operatorname{Pr}(x)$ é o ponto que resolve

$$
\begin{aligned}
\min z & =\sum_{i, j}\left(x_{a_{i j}}-a_{i j}\right)^{2}+\sum_{i, j}\left(x_{b_{i j}}-b_{i j}\right)^{2}+\sum_{i}\left(x_{\pi_{i}}-\pi_{i}\right)^{2} \\
\sum_{j=1}^{N} a_{i j} & =1 \quad \forall i=1, \ldots, N . \\
\sum_{j=1}^{M} b_{i j} & =1 \quad \forall i=1, \ldots, N . \\
\sum_{i=1}^{N} \pi_{i} & =1 \\
a_{i j}, b_{i j}, \pi_{i} & \in \boldsymbol{\theta} .
\end{aligned}
$$

Como o modelo intervalar é consistente, existe ao menos um $\theta$ na caixa $\boldsymbol{\theta}$ que satisfaz as restrições. 
Além disso, o conjunto viável é convexo e o problema tem solução única. Mais ainda, podemos dividir (5.34)-(5.38) em $2 N+1$ problemas da forma

$$
\begin{aligned}
\min z & =\sum_{j}^{N}\left(x_{a_{i j}}-a_{i j}\right)^{2} \\
\sum_{j=1}^{N} a_{i j} & =1 \\
a_{i j}, & \in \boldsymbol{a}_{\boldsymbol{i j}}
\end{aligned}
$$

para $i=1, \ldots, N$,

$$
\begin{aligned}
\min z & =\sum_{j}^{M}\left(x_{b_{i j}}-b_{i j}\right)^{2} \\
\sum_{j=1}^{M} b_{i j} & =1 \\
b_{i j} & \in \boldsymbol{b}_{\boldsymbol{i j}}
\end{aligned}
$$

também para $i=1, \ldots, N$, e

$$
\begin{aligned}
\min z & =\sum_{i}\left(x_{\pi_{i}}-\pi_{i}\right)^{2} \\
\sum_{i=1}^{N} \pi_{i} & =1 \\
\pi_{i} & \in \pi_{\boldsymbol{i}} .
\end{aligned}
$$

O problema da projeção é fácil de resolver em MMOs com mistura Bernoulli. Considere o primeiro subproblema com $i=1$.

$$
\begin{aligned}
\min z & =\left(x_{a_{11}}-a_{11}\right)^{2}+\left(x_{a_{12}}-a_{12}\right)^{2} \\
\sum_{j=1}^{2} a_{1 j} & =1 \\
a_{11} & \in \boldsymbol{a}_{11} \\
a_{12} & \in \boldsymbol{a}_{12} .
\end{aligned}
$$

Fazendo $a_{11}=1-a_{12}$, temos

$$
\begin{aligned}
\min d\left(a_{12}\right) & :=\left(x_{a_{11}}-\left(1-a_{12}\right)\right)^{2}+\left(x_{a_{12}}-a_{12}\right)^{2} \\
a_{12} & \in \boldsymbol{a}_{\mathbf{1 2}} \cap\left[1-\sup \left(a_{11}\right), 1-\inf \left(a_{11}\right)\right] .
\end{aligned}
$$

Agora, nossa função objetivo é $d: \mathbb{R} \rightarrow \mathbb{R}$. Se $\boldsymbol{a}_{12} \cap\left[1-\sup \left(\boldsymbol{a}_{11}\right), 1-\inf \left(\boldsymbol{a}_{11}\right)\right]=\varnothing$ então $\boldsymbol{\theta}$ é inconsistente e não há solução. Caso contrário, expandimos a função $d$ para obter um polinômio de segundo grau em $a_{12}$. O mínimo desse polinômio é dado por

$$
a_{12}^{s}=\frac{1-x_{a_{11}}+x_{a_{12}}}{2} .
$$

Sendo assim, se $a_{12}^{s} \in \boldsymbol{a}_{12} \cap\left[1-\sup \left(\boldsymbol{a}_{11}\right), 1-\inf \left(\boldsymbol{a}_{11}\right)\right]$ então

$$
a_{12}^{*}=\operatorname{argmin}_{x \in \mathcal{M}} d(x)
$$


é a projeção de $x_{a_{12}}$ em $\boldsymbol{\theta}$. O conjunto $\mathcal{M}$ é dado por

$$
\mathcal{M}:=\left\{a_{12}^{s}, \max \left(1-\sup \left(\boldsymbol{a}_{11}\right), \inf \left(\boldsymbol{a}_{\mathbf{1 2}}\right)\right), \min \left(1-\inf \left(\boldsymbol{a}_{\mathbf{1 1}}\right), \sup \left(\boldsymbol{a}_{\mathbf{1 2}}\right)\right)\right\} .
$$

Caso $a_{12}^{s} \notin \boldsymbol{a}_{12} \cap\left[1-\sup \left(\boldsymbol{a}_{11}\right), 1-\inf \left(\boldsymbol{a}_{11}\right)\right]$ então

$$
\mathcal{M}:=\left\{\max \left(1-\sup \left(\boldsymbol{a}_{11}\right), \inf \left(\boldsymbol{a}_{\mathbf{1 2}}\right)\right), \min \left(1-\inf \left(\boldsymbol{a}_{\mathbf{1 1}}\right), \sup \left(\boldsymbol{a}_{\mathbf{1 2}}\right)\right)\right\} .
$$

Mostramos então que para calcular a projeção de um par de coordenadas, basta avaliar a função $d$ em três pontos. É claro que o mesmo raciocínio se aplica às demais distribuições do $M M O_{2,2}$. Para outros modelos com mais parâmetros não conhecemos nenhuma forma tão eficiente.

Ao contrário do Baum-Welch, o método dos gradientes projetados pode ser aplicado a uma sub caixa de $[0,1]^{N^{2}+N M+1}$. Por exemplo, suponha que estamos procurando o máximo de (5.1)-(5.5) em $\boldsymbol{\theta}=(\boldsymbol{A}, \boldsymbol{B}, \boldsymbol{\pi})$, dado por

$$
\boldsymbol{A}=\left(\begin{array}{cc}
{[0.25,0.5]} & {[0.4,0.8]} \\
{[0.3,0.5]} & {[0.4,0.8]}
\end{array}\right), \boldsymbol{B}=\left(\begin{array}{cc}
{[0.1,0.3]} & {[0.6,0.9]} \\
{[0.2,0.4]} & {[0.6,0.8]}
\end{array}\right) \text { e } \boldsymbol{\pi}=\left(\begin{array}{c}
{[0.3,0.5]} \\
{[0.1,0.5]}
\end{array}\right)
$$

O método de gradientes projetados se mantém em $\boldsymbol{\theta}$ a cada iteração. Por outro lado, o algoritmo de Baum-Welch pode deixar a caixa. Adiante comparamos os efeitos dessas duas abordagens no método global.

O algoritmo que implementamos é descrito em Birgin et al. (2000) e é chamado gradientes projetados espectrais. Nessa versão, o iterando é da forma

$$
x_{n+1}=x_{n}+\lambda *\left(\operatorname{Pr}\left(x_{n}-\alpha_{n} \nabla f\left(x_{n}\right)\right)-x_{n}\right) .
$$

O escalar $\lambda$ é chamado coeficiente espectral. Esse método tem as mesmas propriedades do gradientes projetados, mas sua ordem de convergência é superior. Birgin e colaboradores implementam esse algoritmo em FORTRAN e $C$. Nós adaptamos essa última implementação para $C++$, incluindo elementos de programação genérica.

\subsection{Experimentos Numéricos dos Métodos Locais}

Comparamos agora os métodos descritos na seção anterior. Além dos algoritmos de Baum-Welch e SPG, incluímos na análise o método dos pontos interiores, descrito em Wächter e Biegler (2006). Esse é o algoritmo geral de otimização local mais usado atualmente. Para uma descrição detalhada do métodos de pontos interiores, consulte Nocedal e Wright (2006).

Estamos interessados em métodos locais como sub-algoritmos do procedimento global. Portanto, nossa comparação leva em conta a eficiência e o máximo obtido por cada método. Os experimentos abaixo consideram o modelo de Markov oculto com mistura Bernoulli. Essa restrição faz sentido pois os testes do método global também são feitos sobre esse modelo. Além disso não conhecemos um algoritmo eficiente para calcular a projeção $\operatorname{Pr}(x)$ para modelos gerais no SPG.

\subsubsection{Análise dos Métodos}

Considere o $M M O_{2,2}$. Fixado o tamanho da amostra $T$, há $2^{T-1}$ problemas possíveis para esse modelo. Essa redução acontece porque sempre podemos começar a seqüência com 0(veja a seção quebra de simetrias abaixo). Os problemas deste caso são identificados por sua representação decimal. Por exemplo, se $T=5$ e o modelo de mistura emite os sinais 0 e 1 , então a sequiência $O=(00011)$ está associada ao número 3. Na figura abaixo, o eixo das abscissas de cada gráfico identifica o problema resolvido através dessa representação.

Os gráficos mostram o resultado dos algoritmos locais aplicados ao $M M O_{2,2} \operatorname{com} T=10, \ldots, 15$. A esquerda temos o valor da função objetivo em cada instância. A direita apresentamos o tempo para obter a solução, em segundos. 
Nesse experimento, implementamos os algoritmos de Baum-Welch e SPG em Matlab. A função fmincon do pacote de otimização dessa linguagem implementa o método dos pontos interiores. A tolerância em todos os casos é $\epsilon=10^{-6}$. Os três algoritmos são interrompidos após 500 iterações.
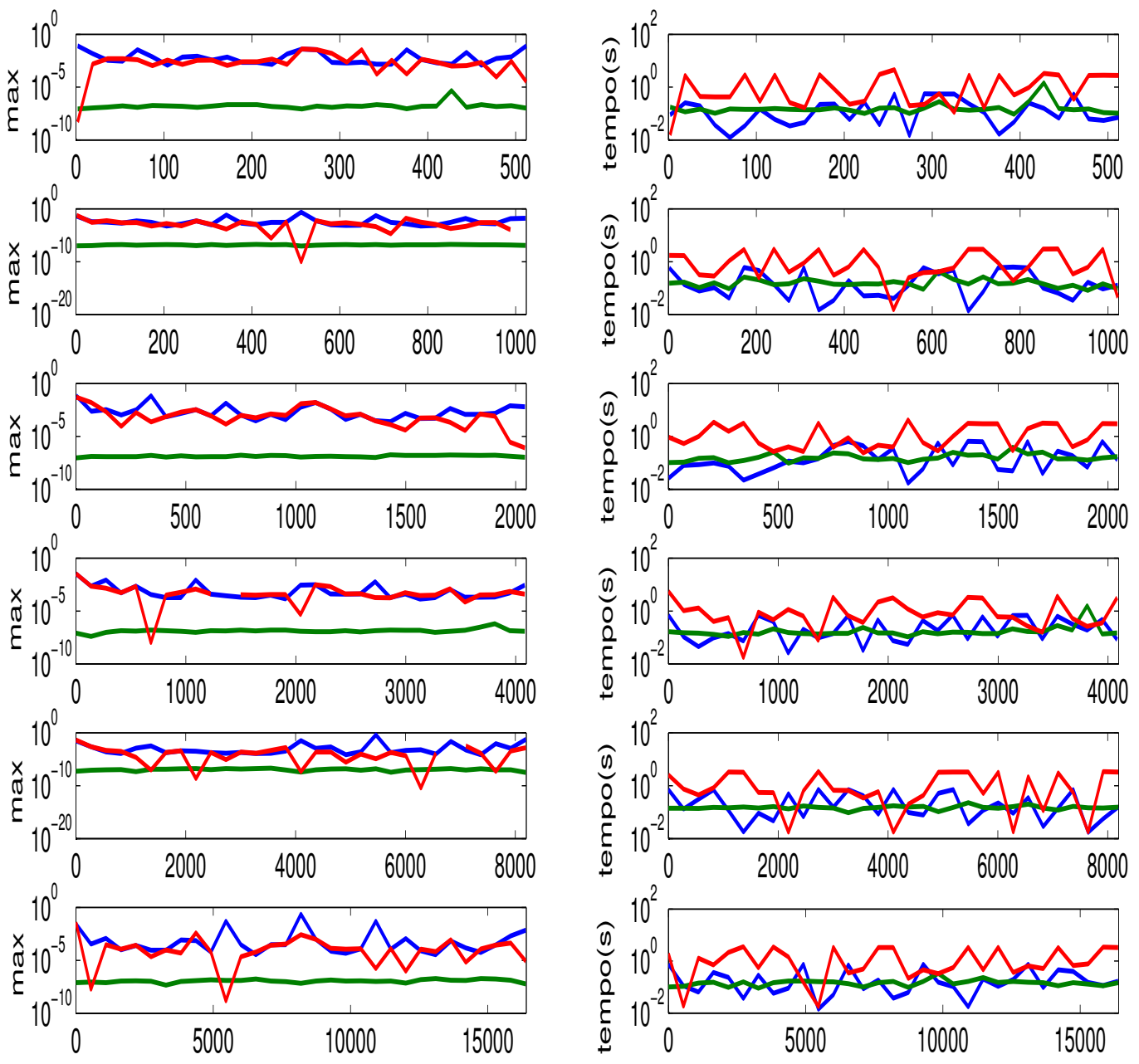

$-B W-\mathbb{P}-S P G$

Figura 5.1: Comparação entre Baum-Welch, SPG e pontos interiores. Todas as instâncias possíveis do $M \mathrm{MO}_{2,2}$ com $\mathrm{T}=10, \ldots, 15$. A esquerda temos o máximo. A direita, o tempo necessário para obter a solução em segundos. Na primeira linha temos os gráficos para $T=10$.

A figura mostra que o método de Baum-Welch é a melhor opção para estimar parâmetros de um $M M O_{2,2}$ quando $T$ é pequeno. Reforçamos essa conclusão com o gráfico 5.3 abaixo. Se considerarmos a qualidade das soluções obtidas, o método de pontos interiores é a pior das três opções. De fato, esse algoritmo não foi o melhor em nenhum dos problemas. O SPG nos dá bons limitantes inferiores para o máximos global, mas seu tempo de execução é o pior dos três, em média.

Realizamos o mesmo experimento com tamanhos amostrais maiores. Para $T=50,60, \ldots, 100$ não é possível listar todos os problemas em tempo hábil. Portanto, dado o número de observações, geramos 100 problemas aleatórios, combinando os sinais 0 e 1. Veja a figura abaixo. 

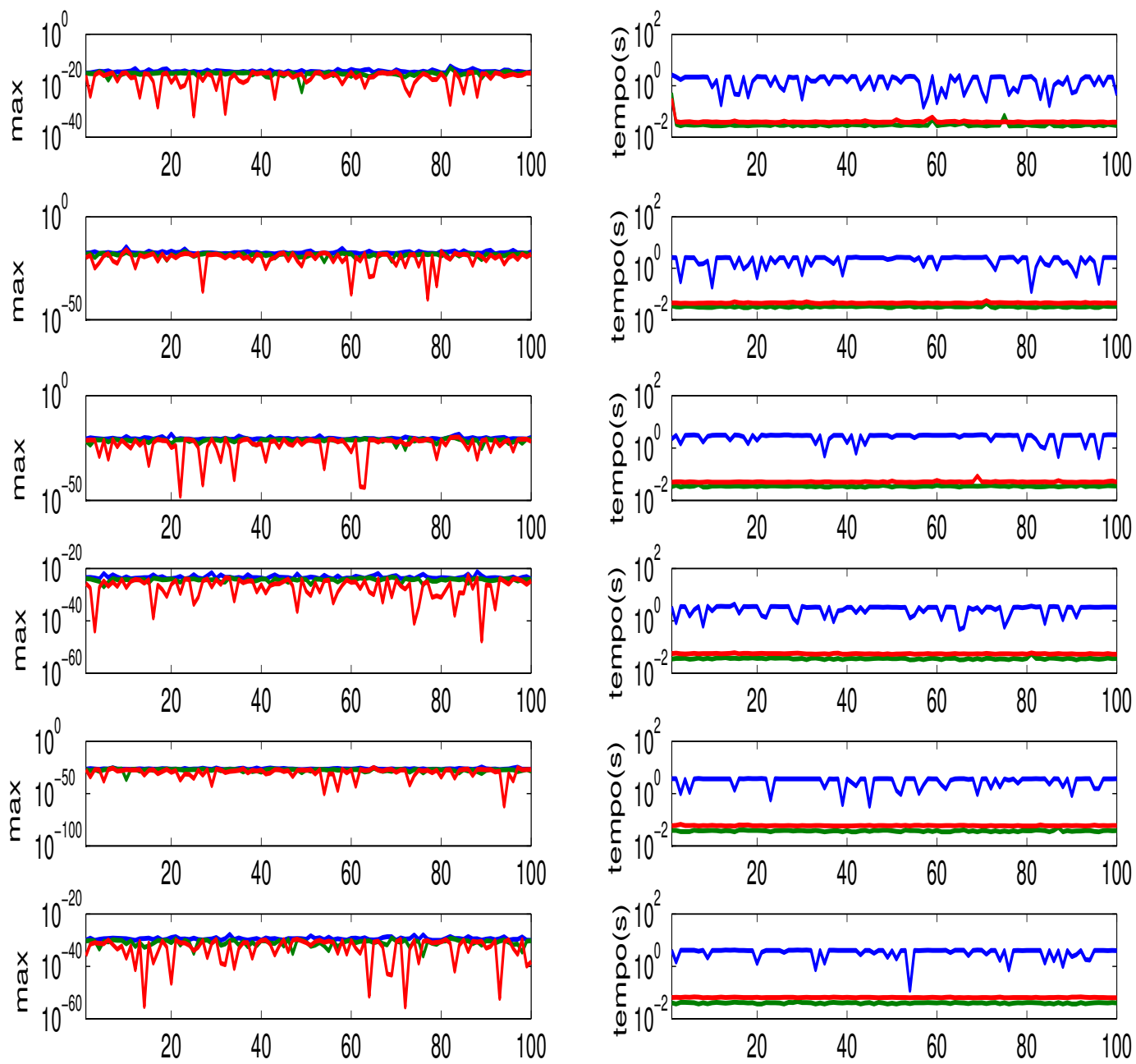

$-B W-\mathbb{P}-S P G$

Figura 5.2: Comparação entre Baum-Welch, SPG e pontos interiores. Instâncias geradas aleatoriamente $M \mathrm{MO}_{2,2}$ com $\mathrm{T}=50,60, \ldots, 100$. A esquerda temos o máximo. A direita, o tempo necessário para obter a solução em segundos. Na primeira linha temos os gráficos para $T=50$.

Nesse caso é mais difícil identificar a melhor opção. O método de pontos interiores é o mais eficiente dos três conforme $T$ aumenta. Em contraste, o algoritmo de Baum-Welch torna-se muito lento. Em algumas instâncias ele leva mais de 4 segundos para determinar a resposta. O SPG tem tempo de execução próximo aos pontos interiores, mas sempre acima deste. Com relação à qualidade dos limitantes inferiores, os gráficos 5.3 e 5.4 deixam claro que o algoritmo de Baum-Welch dá os melhores resultados.

Nosso experimento indica que se $T$ é pequeno, devemos usar o procedimento de Baum-Welch exclusivamente. A escolha do método local fica complicada conforme aumentamos o tamanho da amostra. Em nossa implementação do interval branch and bound testamos duas heurísticas de escolha. Na primeira usamos o método de Baum-Welch exclusivamente. Na segunda, realizamos 200 iterações do SPG e, em seguida, usamos a resposta como entrada para o método de BaumWelch. Com isso procuramos calibrar o ponto inicial do último algoritmo, diminuindo o número de iterações. Caso um máximo local seja localizado nas 200 iterações do SPG então não aplicamos o método de Baum-Welch. Os resultados dessas estratégias serão discutidos ainda neste capítulo, na seção 5.7. 


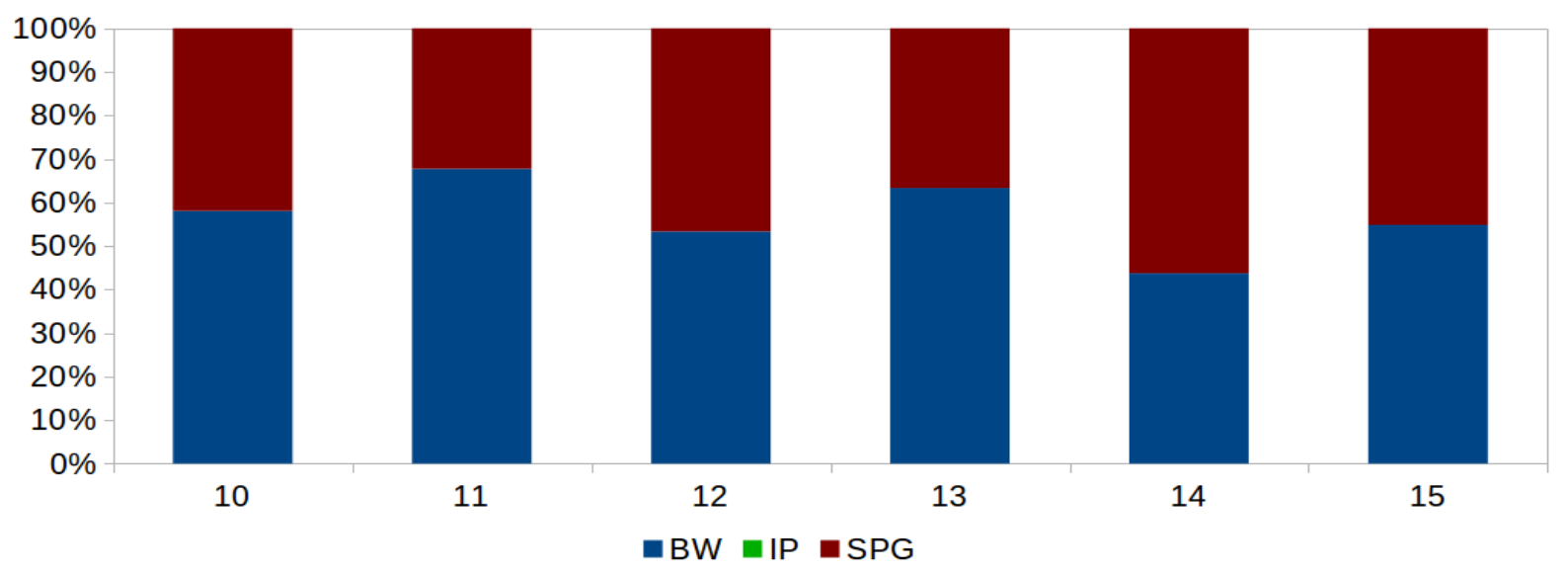

Figura 5.3: Porcentagem de problemas em que cada método encontrou a melhor solução. Todas as instâncias possiveis do $M M_{2,2}$ com $T=10, \ldots, 15$.

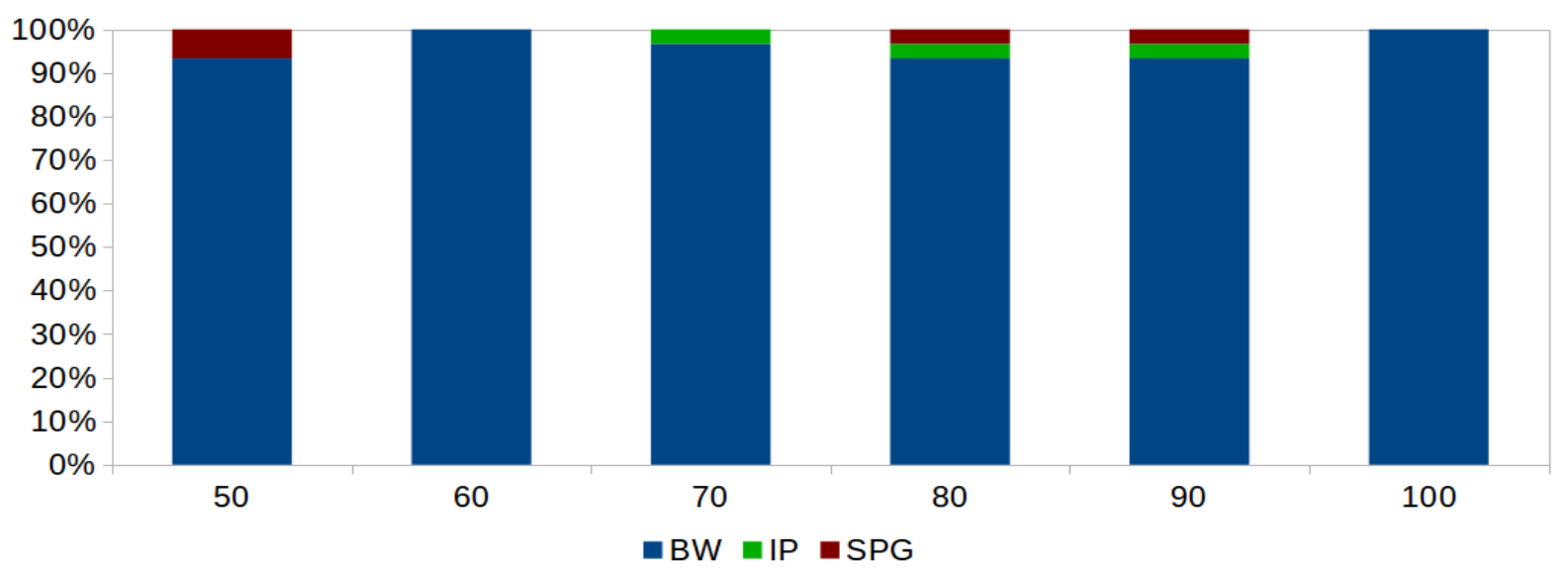

Figura 5.4: Porcentagem de problemas em que cada método encontrou a melhor solução. Resultado de 100 instâncias do $\mathrm{MMO}_{2,2}$ geradas aleatoriamente com $\mathrm{T}=50,60, \ldots, 100$.

\subsubsection{Detectando Máximos Globais}

Testamos agora a capacidade dos métodos locais em determinar o máximo global de (5.1)-(5.5). Considere o $M M_{2,2}$, parametrizado por $\theta=(A, B, \pi)$ onde

$$
\boldsymbol{A}=\left(\begin{array}{ll}
0.1370 & 0.8630 \\
0.0047 & 0.9953
\end{array}\right), \boldsymbol{B}=\left(\begin{array}{ll}
0.5064 & 0.4936 \\
0.8299 & 0.1701
\end{array}\right) \text { e } \boldsymbol{\pi}=\left(\begin{array}{l}
1 \\
0
\end{array}\right)
$$

A partir desse modelo, geramos observações de tamanho 40, 60 e 90. Nosso objetivo é verificar se os métodos locais são capazes de identificar o máximo global e o modelo que gerou a amostra.

O máximo global foi obtido com nossa implementação, descrita na seção 5.4. As tolerâncias nesse experimento são $\epsilon_{X}=1 e-4$ e $\epsilon_{F}=1 e-4$. Cada método local foi testado 50 vezes, a partir de pontos iniciais distintos. Os gráficos abaixo mostram os modelos obtidos em cada execução. O primeiro gráfico de cada figura nos dá os parâmetros $a_{11}$ e $a_{22}$. O segundo apresenta os parâmetros $b_{11}$ e $b_{22}$. O vetor de distribuição inicial é fixado e não faz parte da otimização. $\mathrm{O}$ terceiro gráfico de cada figura nos dá o máximo obtido por cada método ao longo das iterações.

É importante notar que tanto os máximos locais quanto a saída do nosso método foram incapazes de detectar o modelo que gerou os dados. Acreditamos que esse fenômeno mereça atenção. Por outro lado, estuda-lo corretamente foge ao escopo do nosso trabalho. 

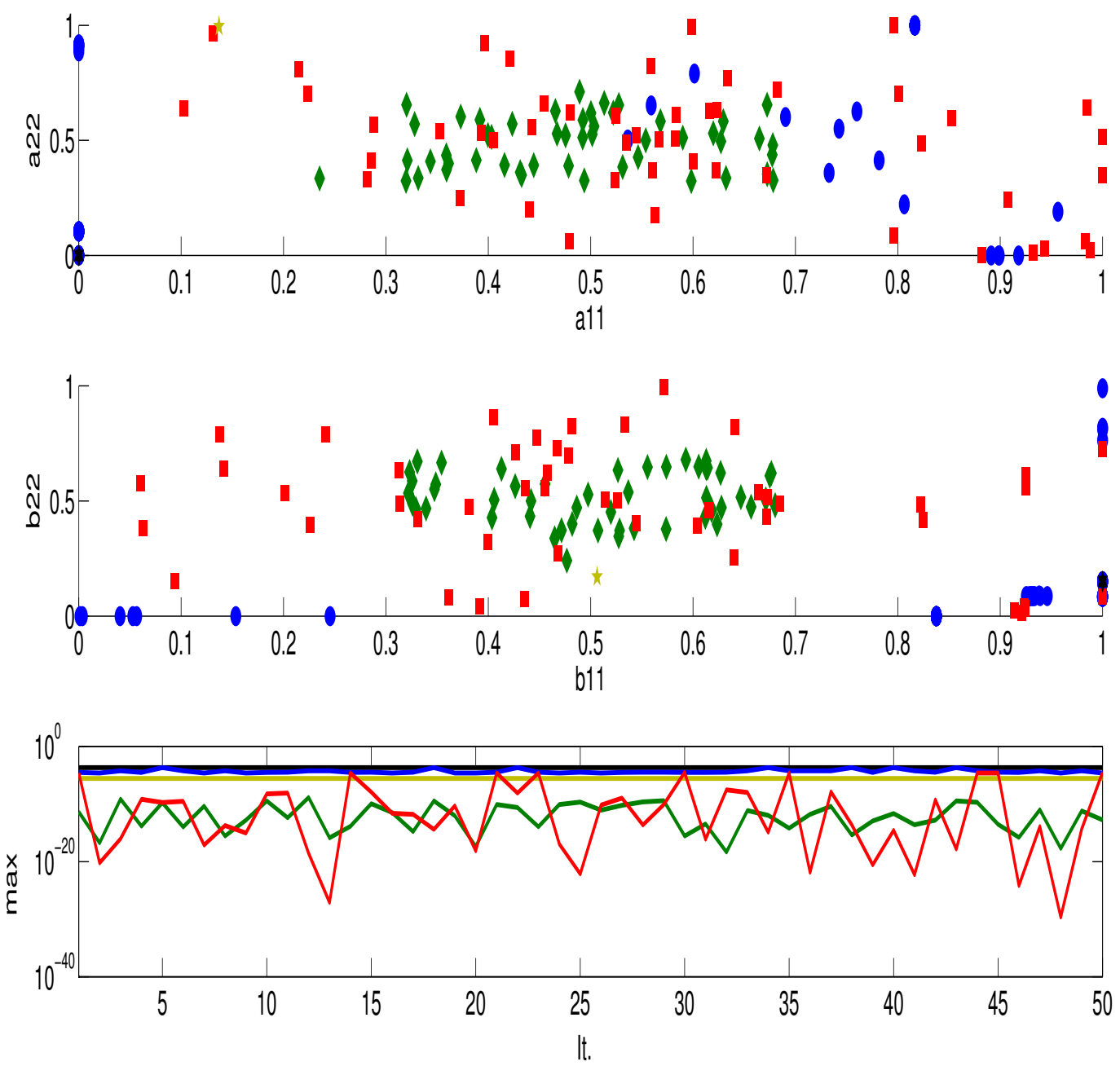

—MODEL 一GLOBAL 一BW - $P$-SPG

Figura 5.5: Dispersão das soluções obtidas com métodos locais, a partir de 50 pontos iniciais distintos. Nos dois primeiros gráficos, temos os estimadores do $\mathrm{MMO}_{2,2}$ com amostra de tamanho 40, gerada a partir do modelo (5.39). O máximo global, obtido com nosso algoritmo e o modelo original também estão representados. O terceiro gráfico nos dá o máximo obtido por cada método local ao longo das iterações.

O conjunto de observações é

$$
O=[1010000000000000100000001000000000000000] .
$$



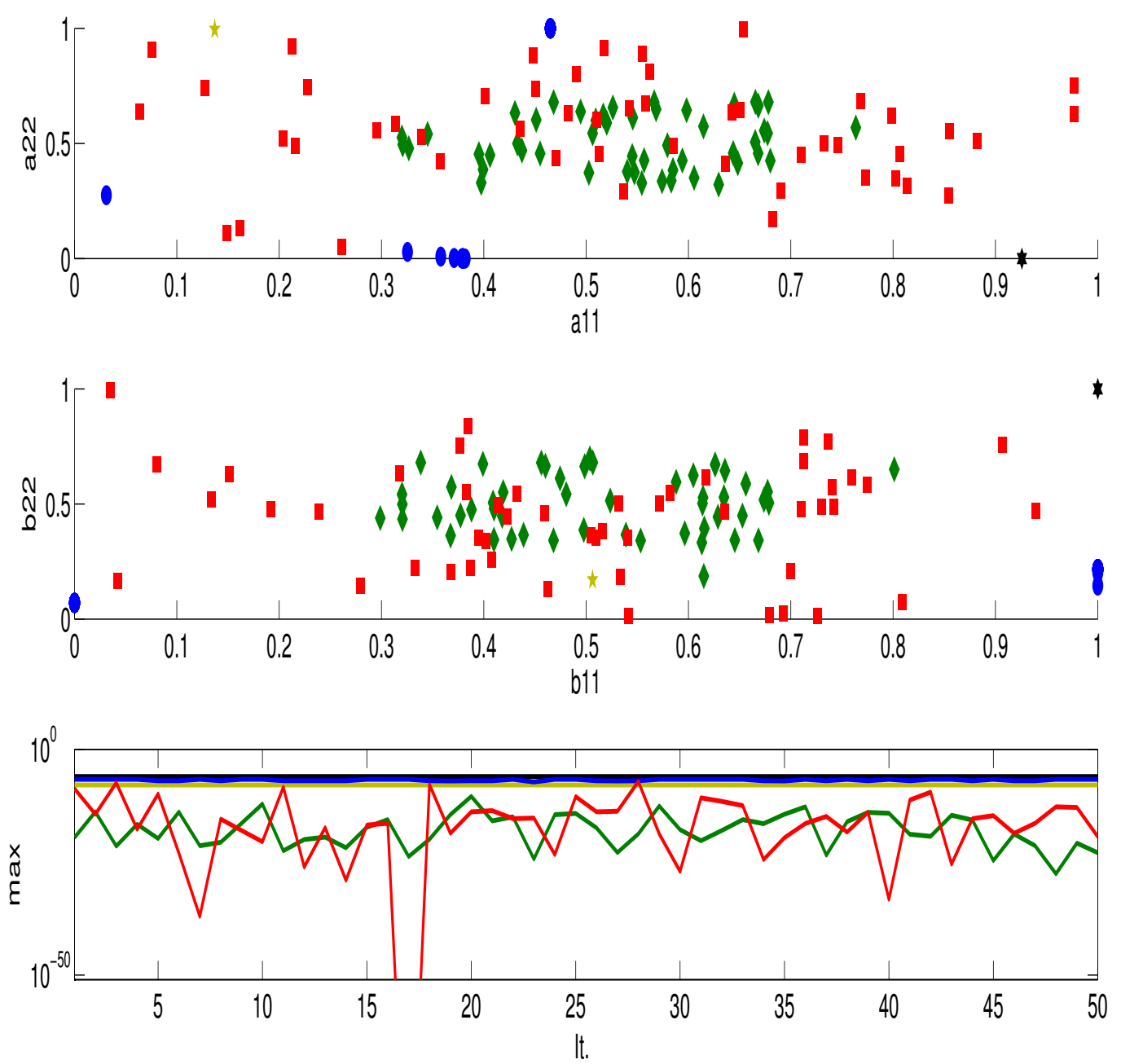

—MODEL 一GLOBAL 一BW - P -SPG

Figura 5.6: Dispersão das soluções obtidas com métodos locais, a partir de 50 pontos iniciais distintos. Nos dois primeiros gráficos, temos os estimadores do $\mathrm{MMO}_{2,2}$ com amostra de tamanho 60, gerada a partir do modelo (5.39). O máximo global, obtido com nosso algoritmo e o modelo original também estão representados. O terceiro gráfico nos dá o máximo obtido por cada método local ao longo das iterações.

O conjunto de observações é

$$
O=[100000010000000000000000010000000000001000000000000000100000] .
$$



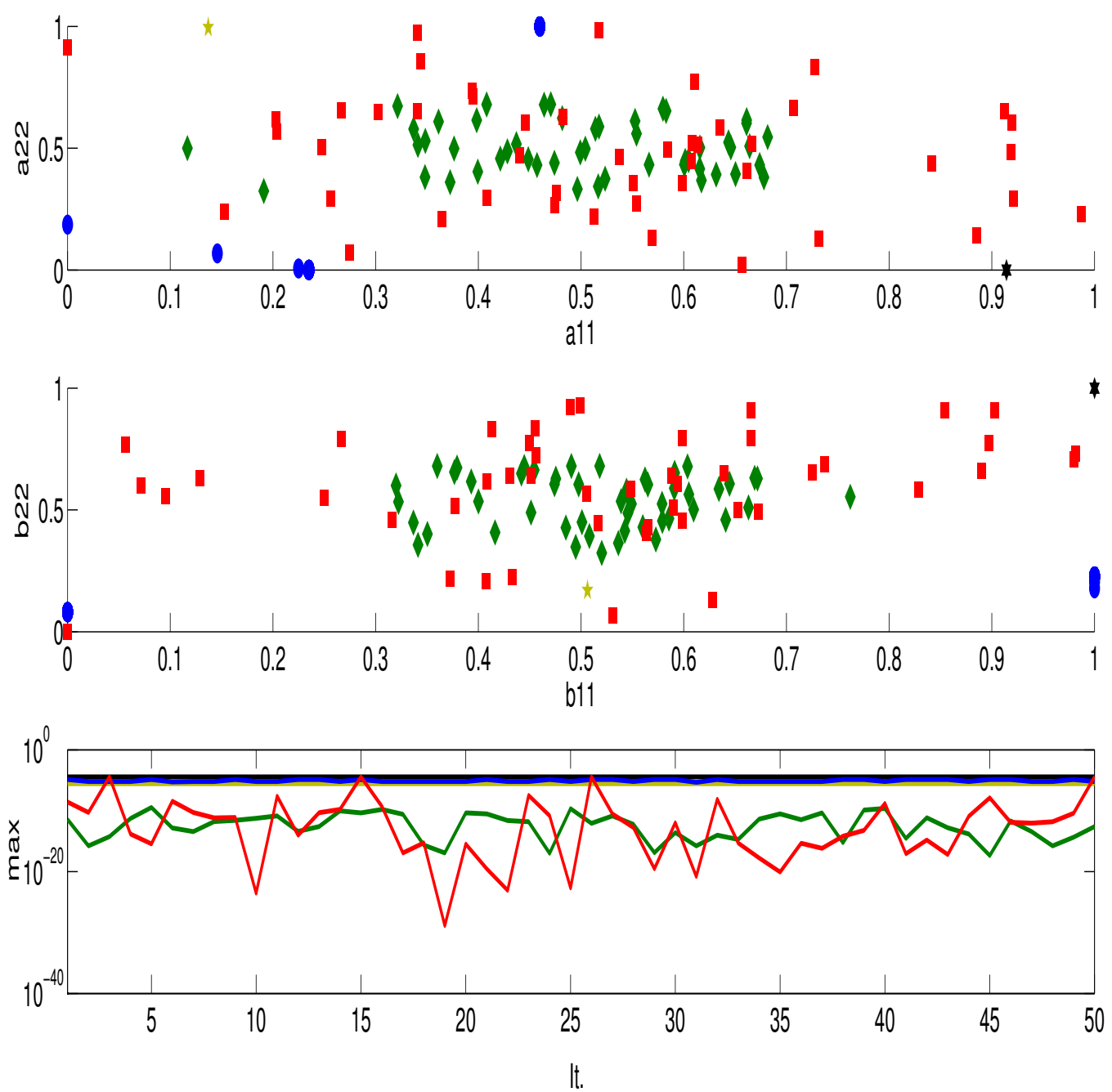

Figura 5.7: Dispersão das soluções obtidas com métodos locais, a partir de 50 pontos iniciais distintos. Nos dois primeiros gráficos, temos os estimadores do $M M_{2,2}$ com amostra de tamanho 90, gerada a partir do modelo (5.39). O máximo global, obtido com nosso algoritmo e o modelo original também estão representados. O terceiro gráfico nos dá o máximo obtido por cada método local ao longo das iterações.

O conjunto de observações é

$$
\begin{aligned}
O= & {[100000100001000000100000000100010000000001000} \\
& 000001001000000000000100000101000000001000000]
\end{aligned}
$$

Dos três experimentos realizados, apenas no primeiro o método de Baum-Welch foi capaz de identificar o máximo global. Os demais algoritmos foram incapazes de localizá-lo. Os métodos de pontos interiores e gradientes projetados espectrais também são mais dispersos do que Baum-Welch. Essa observação sugere que eles são mais sensíveis ao chute inicial.

Esse experimento reforça a conclusão da subseção anterior. O método de Baum-Welch é o algoritmo local mais indicado para estimar parâmetros em MMOs. Entre métodos de pontos interiores e SPG não há um dominante. Os exemplos mostram que em média o SPG encontra máximos locais melhores. Por outro lado, esse método oscila bastante e no segundo exemplo há um caso em que ele encontra uma solução cerca de $10^{40}$ vezes pior do que o método de pontos interiores.

Observe agora que o modelo que gerou os experimentos tem probabilidade de transição muito 
pequena do estado oculto $S_{2}$ para $S_{1}$. Essa pode ser a explicação para o fato de nenhum método ter encontrado o modelo gerador. Nas figuras abaixo repetimos o experimento com $\theta=(A, B, \pi)$ onde

$$
\boldsymbol{A}=\left(\begin{array}{ll}
0.3241 & 0.6759 \\
0.4708 & 0.5292
\end{array}\right), \boldsymbol{B}=\left(\begin{array}{ll}
0.5618 & 0.4382 \\
0.3995 & 0.6005
\end{array}\right) \text { e } \boldsymbol{\pi}=\left(\begin{array}{l}
1 \\
0
\end{array}\right)
$$
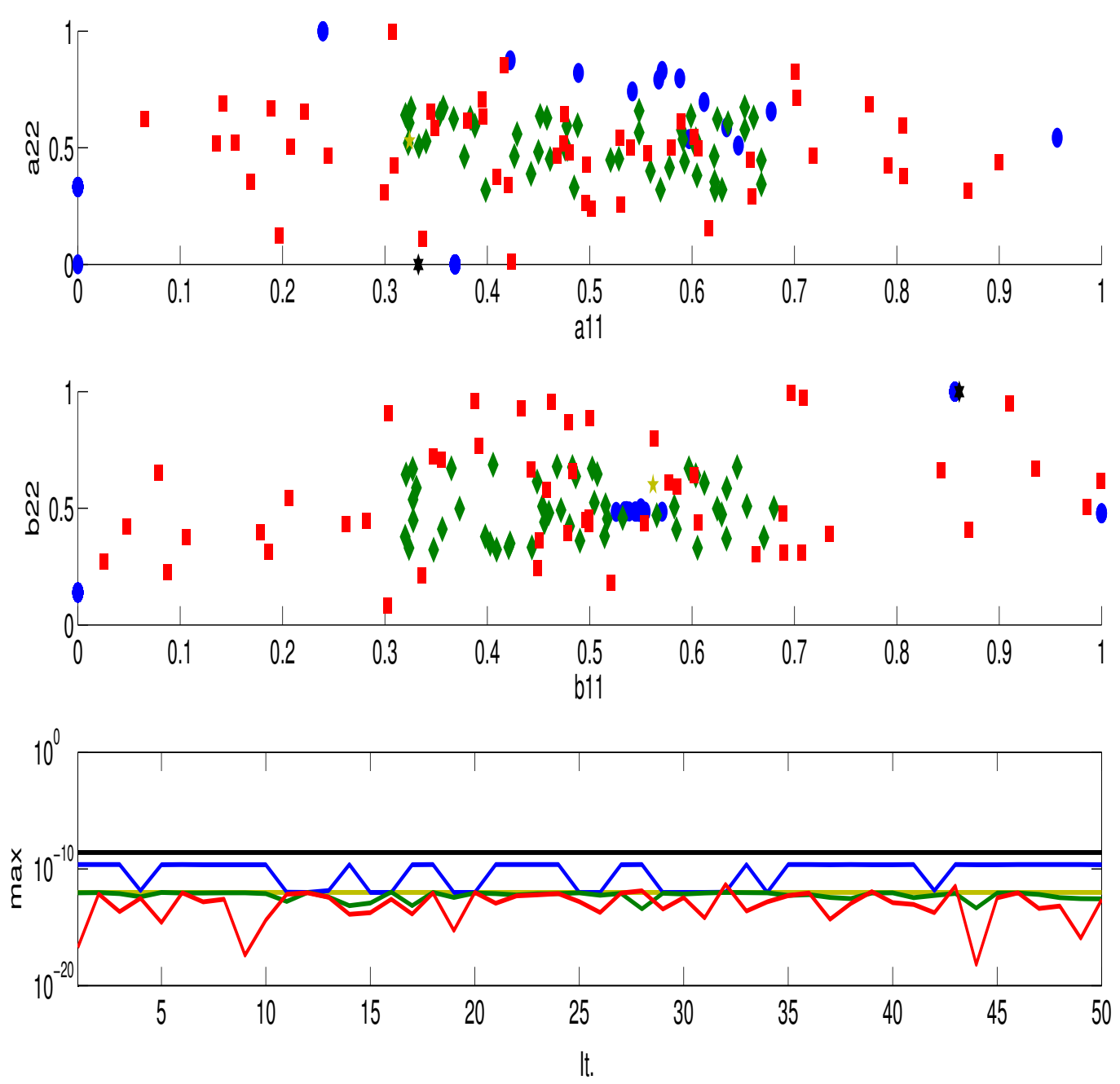

$-M O D E L-G L O B A L-B W-P-S P G$

Figura 5.8: Dispersão das soluções obtidas com métodos locais, a partir de 50 pontos iniciais distintos. Nos dois primeiros gráficos, temos os estimadores do $\mathrm{MMO}_{2,2}$ com amostra de tamanho 40, gerada a partir do modelo (5.40). O máximo global, obtido com nosso algoritmo e o modelo original também estão representados. O terceiro gráfico nos dá o máximo obtido por cada método local ao longo das iterações.

O conjunto de observações é

$$
O=[0101011100101010101110010110010100100010] .
$$



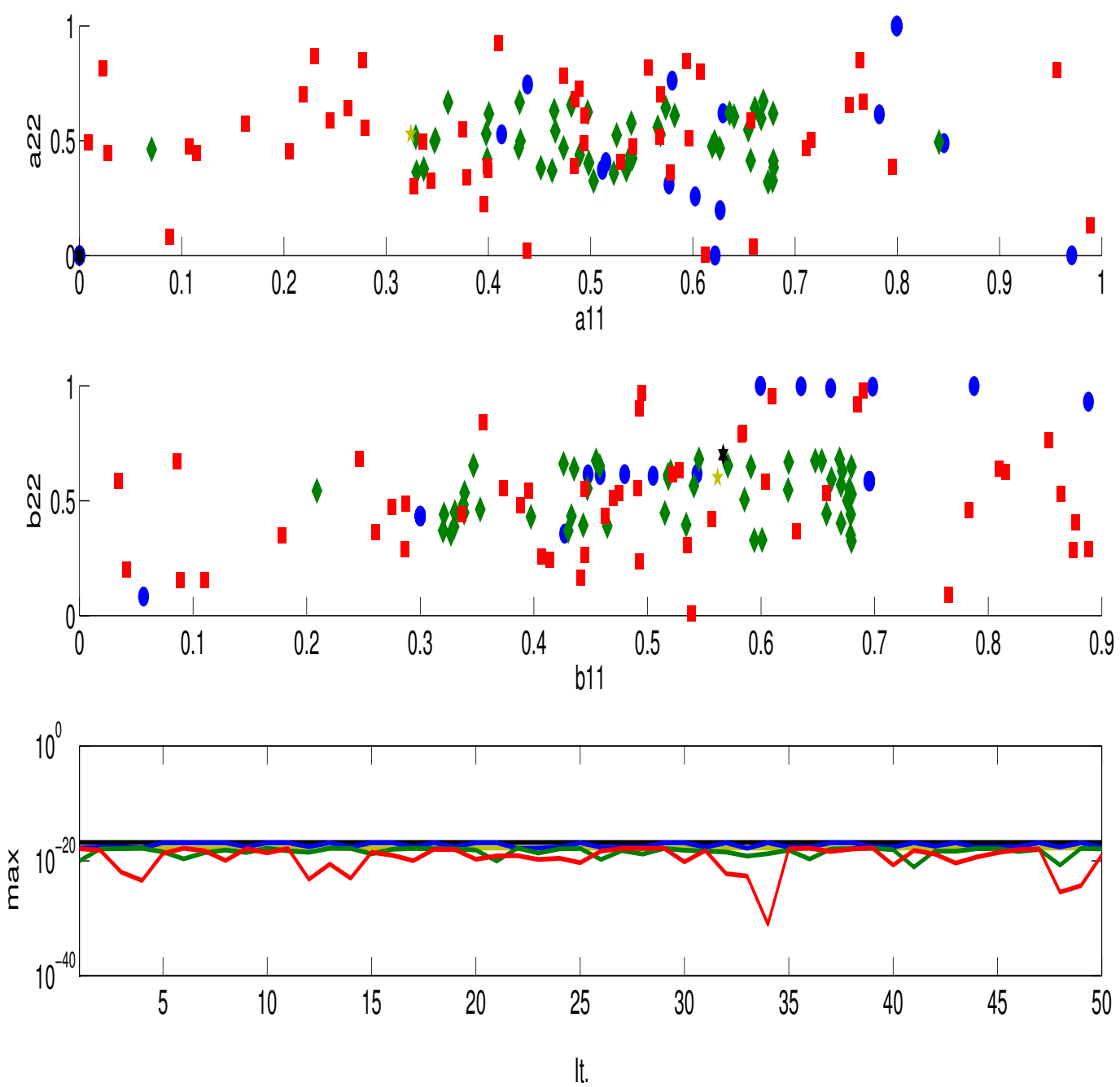

-MODEL 一 GLOBAL 一BW - P -SPG

Figura 5.9: Dispersão das soluções obtidas com métodos locais, a partir de 50 pontos iniciais distintos. Nos dois primeiros gráficos, temos os estimadores do $M M O_{2,2}$ com amostra de tamanho 60, gerada a partir do modelo (5.40). O máximo global, obtido com nosso algoritmo e o modelo original também estão representados. O terceiro gráfico nos dá o máximo obtido por cada método local ao longo das iterações.

O conjunto de observações é

$$
O=[010001011101010001111101101110111001000111001001111101001101] .
$$



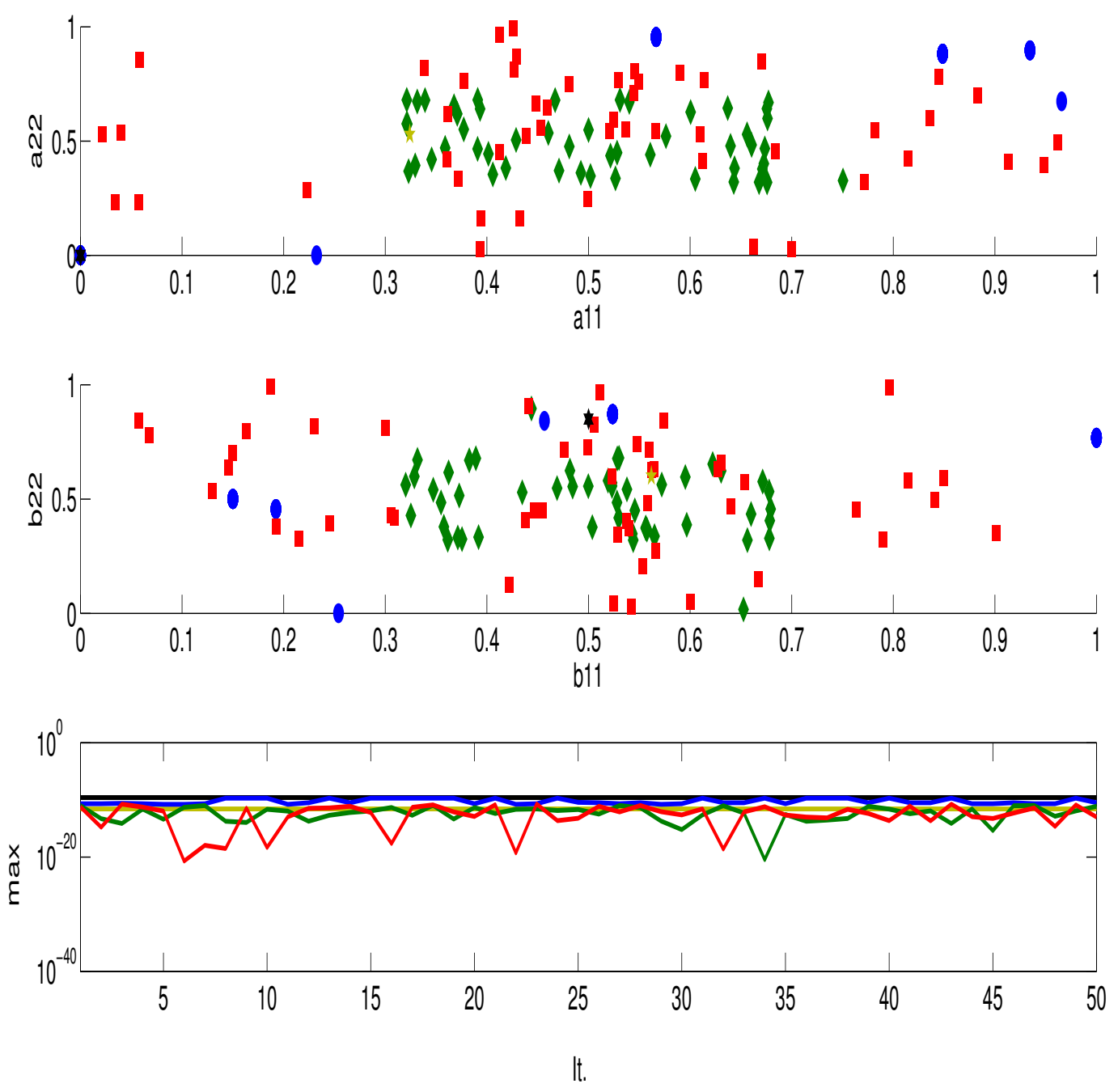

-MODEL 一GLOBAL 一BW - P -SPG

Figura 5.10: Dispersão das soluções obtidas com métodos locais, a partir de 50 pontos iniciais distintos. Nos dois primeiros gráficos, temos os estimadores do $M M O_{2,2}$ com amostra de tamanho 90, gerada a partir do modelo (5.40). O máximo global, obtido com nosso algoritmo e o modelo original também estão representados. O terceiro gráfico nos dá o máximo obtido por cada método local ao longo das iterações.

O conjunto de observações é

$$
\begin{aligned}
O= & {[010100011111111111010101001101010010111110100} \\
& 010010110010011010100111101101110101000010111]
\end{aligned}
$$

Esses testes corroboram a conclusão anterior. O método global e os algoritmos locais não são capazes de identificar o modelo gerador corretamente. Novamente, o máximo global não foi encontrado em nenhuma instância testada. Ao contrário do experimento anterior, o método de pontos interiores é o que melhor aproxima o modelo gerador. 


\subsection{O Algoritmo de Branch and Bound Intervalar}

Nesta seção apresentamos o interval branch and bound, descrito em Kearfott (1996). Ele lida com problemas de otimização global da forma,

$$
\begin{aligned}
\max (\operatorname{glob}) z & =f(x) \\
c(x) & =0 \\
h(x) & \geq 0 \\
x & \in \boldsymbol{x} .
\end{aligned}
$$

O algoritmo realiza uma busca exaustiva em $\boldsymbol{x}$. Para tanto, ele implementa uma árvore de busca e usa cálculo intervalar para excluir ou reduzir caixas que não contém soluções. O método é bastante geral e não exige que $f, g$ ou $h$ sejam diferenciáveis. Por outro lado, a diferenciabilidade das funções permite criar testes que aceleram sua convergência. A forma geral do algoritmo é dada por

Algoritmo 1 (Interval Branch and Bound)

\section{Entradas}

- A caixa inicial $\boldsymbol{x}_{\mathbf{0}}$.

- Extensões intervalares $\boldsymbol{f}, \boldsymbol{c}$ e $\boldsymbol{h}$.

- Um limitante superior do máximo global ub.

\section{Saídas}

- Uma lista $\mathcal{U}$, de caixas com diâmetro pré-estabelecido. Todos os máximos globais de (5.41) estão contidos em pelo menos uma caixa de $\mathcal{U}$.

\section{Passos do Algoritmo}

1. Inicie uma lista $\mathcal{L} \leftarrow \boldsymbol{x}_{0}$;

2. Enquanto $\mathcal{L} \neq \varnothing$ faça:

(a) Remova uma caixa $\boldsymbol{x}$ de $\mathcal{L}$;

(b) Processe $\boldsymbol{x}$

i. Verifique se $\boldsymbol{x}$ pode ser eliminada. Se sim, descarte-a e volte para 2a;

ii. Reduza $\boldsymbol{x}$, excluindo regiões que não podem conter máximos globais;

iii. Atualize a estimativa do limitante superior ub;

$i v$. Se $\boldsymbol{x}$ atende aos critérios de aceitação, guarde-a em $\mathcal{U}$ e volte para 2a;

v. Divida $\boldsymbol{x}$ em sub-caixas $\boldsymbol{x}_{\mathbf{1}}, \ldots, \boldsymbol{x}_{\boldsymbol{q}}$;

(c) Insira as caixas obtidas no passo $2 b$ em $\mathcal{L}$;

FIM - Enquanto

Para aceitar uma caixa como solução, usamos dois critérios descritos em Hansen e Walster (2004). Dados $\epsilon_{X}>0$ e $\epsilon_{F}>0$, uma caixa $\boldsymbol{x}^{*}$ é solução de (5.41) se $\boldsymbol{x}^{*}$ tem ao menos um ponto viável e

$$
w\left(\boldsymbol{x}^{*}\right)<\epsilon_{X} \text { e } u b-i n f\left(\boldsymbol{f}\left(\boldsymbol{x}^{*}\right)\right)<\epsilon_{F} * u b .
$$

Em geral, provar a existência de pontos viáveis em $\boldsymbol{x}^{*}$ é um problema difícil. Novamente, a estrutura das restrições (5.2)-(5.5) facilitam a tarefa. Diremos que a caixa $\boldsymbol{\theta}^{*}=(\boldsymbol{A}, \boldsymbol{B}, \boldsymbol{\pi})$ tem ao 
menos um ponto viável se

$$
\begin{aligned}
& 1 \in \sum_{j=1}^{N} \boldsymbol{a}_{\boldsymbol{i j}} \quad \forall i=1, \ldots, N, \\
& 1 \in \sum_{j=1}^{M} \boldsymbol{b}_{\boldsymbol{i j}} \quad \forall i=1, \ldots, N \mathrm{e} \\
& 1 \in \sum_{i=1}^{N} \boldsymbol{\pi}_{\boldsymbol{i}} .
\end{aligned}
$$

Segundo Kearfott (1996), o interval branch and bound é finito se a seqüência de estimativas de $u b$ for decrescente. Além disso esse é um algoritmo conservador. Ao final do processo, todos os máximos globais estão contidos em pelo menos uma caixa de $\mathcal{U}$. Por outro lado, nem toda caixa em $\mathcal{U}$ contém necessariamente um máximo global.

Há várias heurísticas de divisão de caixas. A estratégia mais comum é a bisseção na direção de maior diâmetro. Dada uma caixa $\boldsymbol{x}$ de dimensão $n$, a bisseção consiste no algoritmo a seguir.

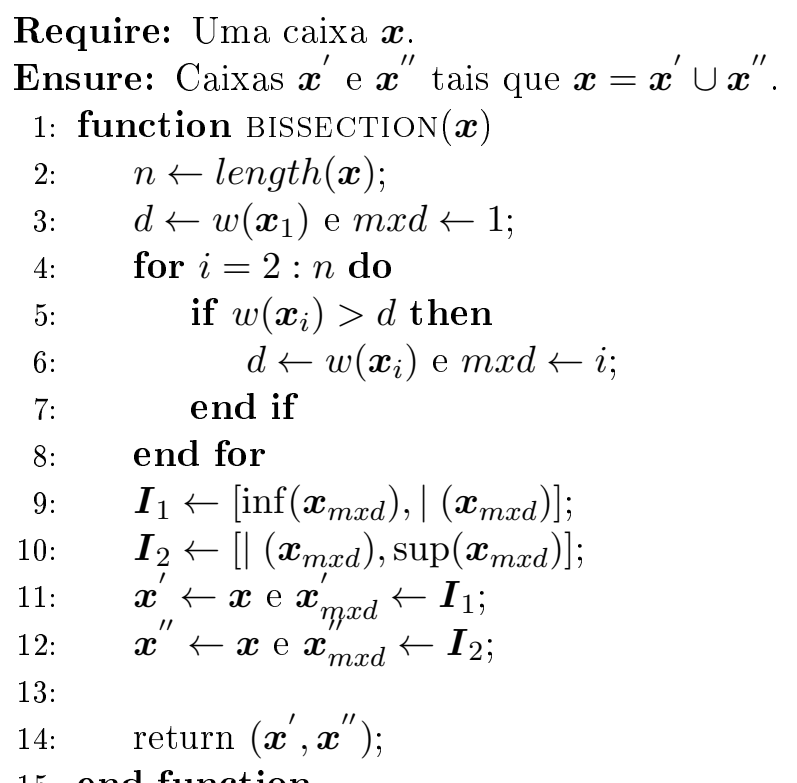

15: end function

É claro que esse algoritmo pode ser generalizado. A generalização mais comum é a trisseção, onde a direção com maior diâmetro é dividida em três. Essa abordagem aumenta o número de caixas em $\mathcal{L}$, mas evita o efeito de agrupamento, descrito em Moore et al. (2009). Quando a divisão da caixa contém um máximo global, as duas caixas resultantes passam a ter essa solução. Esse fenômeno diminui a eficiência do algoritmo.

Em nossos experimentos não observamos agrupamento das soluções. Dessa forma, adotamos a bisseção como padrão em nosso algoritmo. No entanto, a estratégia de trisseção também está disponível em nossa implementação. Para outras formas de divisão de caixas, consulte Pedamallu et al. (2008) e Kearfott (1996).

Sobre a seleção da caixa que será processada, Hansen e Walster (2004) sugerem que nos casos de maximização, a caixa com maior limitante superior do máximo global seja escolhida. Essa ideia exige que os elementos na árvore de busca sejam guardados como um par $\left(u b_{\boldsymbol{x}}, \boldsymbol{x}\right)$.

Em nosso trabalho seguimos a estratégia de seleção proposta por Hansen. Ela garante que na árvore de busca, o limitante superior dos nós pai são sempre maiores ou iguais aos dos filhos. Dessa forma temos uma maneira simples de atualizar $u b$ a cada iteração do algoritmo. Basta tomar

$$
u b=\max _{\boldsymbol{x} \in \mathcal{U}}\left(u b_{\boldsymbol{x}}\right) .
$$




\subsubsection{Exclusão e Redução de Caixas}

Uma parte importante do interval branch and bound são os testes de exclusão de caixas. A seguir, apresentamos três procedimentos típicos em aritmética intervalar para essa finalidade. Derivamos também um novo teste a partir das condições KKT do problema.

Os testes mais comuns para excluir caixas em resolvedores de aritmética intervalar são

1. Viabilidade. Se $0 \notin \boldsymbol{c}(\boldsymbol{x})$ então $\boldsymbol{x}$ não contém pontos viáveis e pode ser descartada.

2. Ponto médio. Se $x^{*}$ é um ponto viável então excluímos $\boldsymbol{x}$ se $f\left(x^{*}\right)>\sup (\boldsymbol{f}(\boldsymbol{x}))$.

3. Propagação das restrições.

No nosso caso o teste de viabilidade é o seguinte. Se $\boldsymbol{\theta}=(\boldsymbol{A}, \boldsymbol{B}, \boldsymbol{\pi})$ então excluímos $\boldsymbol{\theta}$ se qualquer uma das relações abaixo não é satisfeita.

$$
\begin{aligned}
1 \in \sum_{j=1}^{N} \boldsymbol{a}_{\boldsymbol{i} j} & \forall i=1, \ldots, N, \\
1 \in \sum_{j=1}^{M} \boldsymbol{b}_{i j} & \forall i=1, \ldots, N \mathrm{e} \\
1 \in \sum_{i=1}^{N} \boldsymbol{\pi}_{\boldsymbol{i}} . &
\end{aligned}
$$

Para realizar o teste do ponto médio, precisamos conhecer ao menos um ponto viável do problema. Em nossa implementação, usamos os algoritmos locais discutidos em 5.2 para obter esses pontos. Antes de entrar no laço principal do interval branch and bound, calculamos um limitante inferior $l b$ do máximo global. A cada iteração esse limitante é atualizado e em seguida realizamos o teste do ponto médio.

A propagação de restrições é a mesma descrita em 3.3.2. Se o conjunto de restrições é dado por

$$
\begin{aligned}
x_{1}+\ldots+x_{n} & =1 \\
x_{i} & \in \boldsymbol{x}_{\boldsymbol{i}}
\end{aligned}
$$

então, aplicando as regras de cálculo da aritmética intervalar temos

$$
\boldsymbol{x}_{\boldsymbol{i}}{ }^{\prime}=\left(1-\sum_{\substack{j=1 \\ j \neq i}}^{n} \boldsymbol{x}_{\boldsymbol{i}}\right) \cap \boldsymbol{x}_{\boldsymbol{i}} .
$$

Se $\boldsymbol{x}_{\boldsymbol{i}}{ }^{\prime}$ é o conjunto vazio então não há pontos viáveis em $\boldsymbol{x}_{\boldsymbol{i}}$ e eliminamos a caixa. Caso contrário, $\boldsymbol{x}_{\boldsymbol{i}}{ }^{\prime}$ é intervalo pelo menos tão estreito quanto $\boldsymbol{x}_{\boldsymbol{i}}$. A propagação de restrições consiste em aplicar essa relação a todos os índices $i$, enquanto houver redução de $\boldsymbol{x}_{\boldsymbol{i}}$. Apresentamos o algoritmo de propagação de restrições para MMOs na seção 3.3.2.

Concluímos a seção apresentando um novo teste para exclusão e redução de caixas, baseado nas condições KKT do problema. Se $\theta^{*}$ é solução de (5.1)-(5.5) então

$$
\begin{aligned}
\frac{\partial L}{\partial a_{i j}}\left(\theta^{*}\right)-\lambda_{A_{i}}^{*}-\mu_{a_{i j}}^{*} & =0 \\
\frac{\partial L}{\partial a_{i k}}\left(\theta^{*}\right)-\lambda_{A_{i}}^{*}-\mu_{a_{i k}}^{*} & =0 .
\end{aligned}
$$


Subtraindo a primeira equação da segunda temos

$$
\frac{\partial L}{\partial a_{i j}}\left(\theta^{*}\right)-\frac{\partial L}{\partial a_{i j}}\left(\theta^{*}\right)-\mu_{a_{i j}}^{*}+\mu_{a_{i k}}^{*}=0 .
$$

Além disso, as condições de complementaridade afirmam que

$$
\mu_{a_{i j}}^{*} a_{i j}^{*}=0 \text { e } \mu_{a_{i k}}^{*} a_{i k}^{*}=0 .
$$

O resultado abaixo segue imediatamente dessas equações.

Proposição 5 Sejam $\boldsymbol{\theta}=(\boldsymbol{A}, \boldsymbol{B}, \boldsymbol{\pi})$ e $\theta^{*} \in \boldsymbol{\theta}$ uma solução de (5.1)-(5.5). Se $0 \notin \boldsymbol{a}_{\boldsymbol{i j}}$ e $0 \notin \boldsymbol{a}_{\boldsymbol{i k}}$ então

$$
\frac{\partial L}{\partial a_{i j}}\left(\theta^{*}\right)=\frac{\partial L}{\partial a_{i j}}\left(\theta^{*}\right)
$$

Segue daí que se

$$
\frac{\partial L}{\partial a_{i j}}(\boldsymbol{\theta}) \cap \frac{\partial L}{\partial a_{i j}}(\boldsymbol{\theta})=\varnothing
$$

então podemos descartar $\boldsymbol{\theta}$. É claro que o teste se aplica às variáveis $b_{i j}$ e $\pi_{i}$.

Além do teste de exclusão, podemos usar as condições KKT para reduzir uma caixa $\boldsymbol{\theta}$. Seja

$$
\boldsymbol{g}=\frac{\partial L}{\partial a_{i j}}(\boldsymbol{\theta})-\frac{\partial L}{\partial a_{i j}}(\boldsymbol{\theta})
$$

as relações abaixo decorrem da equação (5.42) e das condições de complementaridade

1. Se $\inf (\boldsymbol{g})>0$ então $\mu_{i j}>0$ e $a_{i j}=0$.

2. Se $\sup (\boldsymbol{g})<0$ então $\mu_{i k}>0$ e $a_{i k}=0$.

Todos os testes apresentados nesta seção são eficientes. Eles dependem apenas da função objetivo e seu vetor gradiente. Na seção 5.6 discutimos métodos de segunda ordem para redução de caixas.

\subsection{Quebra de Simetria}

Considere o $M M O_{2,4}$, parametrizado por $\theta=(A, B, \pi)$ tais que

$$
\boldsymbol{A}=\left(\begin{array}{cc}
0.3 & 0.7 \\
0.6 & 0.4
\end{array}\right), \boldsymbol{B}=\left(\begin{array}{cccc}
0.25 & 0.2 & 0.3 & 0.25 \\
0.05 & 0.1 & 0.4 & 0.45
\end{array}\right) \text { e } \boldsymbol{\pi}=\left(\begin{array}{c}
0.4 \\
0.6
\end{array}\right)
$$

Se $O=(1,4,2,3,3,4,4,2,1,3)$ é uma seqüência de observações, então $\mathbb{P}(O \mid \theta)=7.6892 e-07$. Agora considere outro $M M O_{2,4}$, parametrizado por $\theta^{*}=\left(A^{*}, B^{*}, \pi^{*}\right)$ com

$$
\boldsymbol{A}^{*}=\left(\begin{array}{cc}
0.4 & 0.6 \\
0.7 & 0.3
\end{array}\right), \boldsymbol{B}^{*}=\left(\begin{array}{cccc}
0.05 & 0.1 & 0.4 & 0.45 \\
0.25 & 0.2 & 0.3 & 0.25
\end{array}\right) \text { e } \boldsymbol{\pi}^{*}=\left(\begin{array}{c}
0.6 \\
0.4
\end{array}\right)
$$

Novamente, aplicando a recursão backward temos $\mathbb{P}\left(O \mid \theta^{*}\right)=7.6892 e-07$.

O resultado será o mesmo para qualquer seqüência de observações. Isto acontece porque a única diferença entre os modelos é a permutação entre linhas de colunas na matriz de transição e a troca de linhas na emissão. Nesta seção provamos que o cálculo da verossimilhança dos MMOs é invariante com relação a permutações. Mostramos também como eliminar simetrias durante a execução do interval branch and bound. Começamos com a seguinte definição.

Definição 15 Dois $M M O s$ parametrizados por $\theta$ e $\theta^{*}$ são simétricos se para qualquer conjunto de observações $O, L_{O}(\theta)=L_{O}\left(\theta^{*}\right)$. 
O lema abaixo é demonstrado em livros de álgebra linear e análise numérica. Veja por exemplo Issacson e Keller (1994).

Lema 1 Seja $M \in \mathbb{R}^{n x m}$ e $e_{k}$ o k-ésimo vetor canônico. Se E é uma matriz de permutação então

$$
\operatorname{diag}\left(E M e_{k}\right)=\operatorname{Ediag}\left(M e_{k}\right) E^{\prime} .
$$

Com esse resultado, provamos o teorema a seguir.

Teorema 6 Seja E uma matriz de permutação. Se $\theta$ e $\theta^{*}$ são parametrizaçôes de MMOs tais que $A^{*}=E A E^{\prime}, B^{*}=E B$ e $\pi^{*}=E \pi$ então os modelos são simétricos.

Demonstração - Pelo resultado anterior e considerando a definição de $\theta^{*}$ temos

$$
L_{O}\left(\theta^{*}\right)=\pi^{*} E^{\prime} E P\left(o_{1}\right) E^{\prime} E A E^{\prime} \ldots E A E^{\prime} E P\left(o_{T}\right) E^{\prime} \overrightarrow{1} .
$$

Observando que $E E^{\prime}=I$ e $E^{\prime} \overrightarrow{1}=\overrightarrow{1}$, segue o resultado

O teorema diz que permutações nos estados ocultos não alteram a função de verossimilhança. Observe que no exemplo acima, $\theta^{*}$ é obtido a partir de $\theta$ com a permutação

$$
E=\left(\begin{array}{ll}
0 & 1 \\
1 & 0
\end{array}\right)
$$

O interval branch and bound é bastante sensivel a simetrias. Por realizar busca exaustiva no domínio, quanto mais máximos globais o problema tiver, menor será a eficiência do algoritmo. Para lidar com esse problema, faremos duas suposições.

1. O simbolo com maior número de emissões é o 1 , o segundo simbolo com maior número de emissões é o 2 e assim por diante.

2. O estado oculto 1 tem probabilidade maior de emitir 1 do que os demais estados.

A primeira suposição não é restritiva. Dado o conjunto de observações $O$, basta renomear os símbolos para atender essa exigência. Em caso de empate no número de emissões, o simbolo menor recebe o rótulo menor. Em nossa implementação, esse processo é realizado antes de começarmos o interval branch and bound. Depois de concluído, o algoritmo troca as colunas das soluções de acordo com a troca dos símbolos para obter a resposta correta. No exemplo acima, a seqüência $O$ nos dá

$$
O^{*}=(3,2,4,1,1,2,2,4,3,1) .
$$

Se

$$
\boldsymbol{A}=\left(\begin{array}{ll}
0.4935 & 0.5065 \\
0.0000 & 1.0000
\end{array}\right), \boldsymbol{B}=\left(\begin{array}{llll}
0.0000 & 0.1833 & 0.8167 & 0.0000 \\
0.3324 & 0.3126 & 0.1334 & 0.2216
\end{array}\right) \text { e } \boldsymbol{\pi}=\left(\begin{array}{c}
0.4 \\
0.6
\end{array}\right)
$$

maximiza $L_{O^{*}}(\theta)$ então

$$
\boldsymbol{A}=\left(\begin{array}{ll}
0.4935 & 0.5065 \\
0.0000 & 1.0000
\end{array}\right), \boldsymbol{B}=\left(\begin{array}{llll}
0.8167 & 0.0000 & 0.0000 & 0.1833 \\
0.1334 & 0.2216 & 0.3324 & 0.3126
\end{array}\right) \text { e } \boldsymbol{\pi}=\left(\begin{array}{c}
0.4 \\
0.6
\end{array}\right)
$$

maximiza $L_{O}(\theta)$.

A segunda hipótese implica que

$$
b_{11} \geq b_{i 1} \quad i=2, \ldots, N .
$$

Dessa forma, durante o processo de busca excluímos caixas que satisfazem

$$
\sup \left(\boldsymbol{b}_{\mathbf{1 1}}\right)<\inf \left(\boldsymbol{b}_{\boldsymbol{i 1}}\right) \quad i=2, \ldots, N .
$$


No exemplo acima, nosso algoritmo descartaria o ponto $\theta^{*}$. No entanto seria fácil recuperá-lo conhecendo $\theta$ e as permutações possíveis entre os estados ocultos.

\subsection{Aceleração da Convergência}

Nesta seção apresentamos métodos para redução e exclusão de caixas. Os dois recursos implementados em nosso interval branch and bound são o método de Newton intervalar e a propagação da função objetivo. Além disso, discutimos o teorema de Moore que nos dá condições suficientes para que uma caixa $\boldsymbol{x}$ tenha pelo menos uma raíz da função $f: \mathbb{R}^{n} \rightarrow \mathbb{R}^{n}$.

\subsubsection{Método de Newton}

Seja $f: \mathbb{R}^{n} \rightarrow \mathbb{R}^{n}$ diferenciável na caixa $\boldsymbol{x}_{\mathbf{0}}$ e denote por $J$ a matriz jacobiana da função. O método de Newton é usado para limitar o conjunto que contém todas as raízes de $f$ em $\boldsymbol{x}_{\mathbf{0}}$. Considere as extensões intervalares $\boldsymbol{f}$ e $\boldsymbol{J}$. Se $z, y \in \boldsymbol{x}_{\mathbf{0}}$ e $f(y)=0$ então, pelo teorema do valor médio intervalar (2.8) temos

$$
0=f(y) \in \boldsymbol{f}\left(\boldsymbol{z}^{I}\right)+\boldsymbol{J}\left(\boldsymbol{x}_{\mathbf{0}}\right)(y-z)
$$

onde $\boldsymbol{z}^{I}$ é o intervalo degenerado $[z, z]$. Segue daí que fixado $z \in \boldsymbol{x}_{\mathbf{0}}$, o conjunto

$$
S_{z}:=\left\{w \in \boldsymbol{x}_{\mathbf{0}} \mid \exists f^{*} \in \boldsymbol{f}\left(x^{I}\right), J^{*} \in \boldsymbol{J}\left(\boldsymbol{x}_{\mathbf{0}}\right) \text { e } f^{*}+J^{*}(w-x)=0\right\}
$$

contém todos os pontos $y \in \boldsymbol{x}_{\mathbf{0}}$ tais que $f(y)=0$. Esse resultado não é independente do $z$ escolhido.

O método de Newton intervalar gera uma seqüência de caixas tais que

$$
\boldsymbol{x}_{\mathbf{0}} \supseteq \boldsymbol{x}_{\mathbf{1}} \supseteq \ldots \supseteq S_{z} .
$$

Cada caixa é da forma

$$
\boldsymbol{x}_{n+1}=\boldsymbol{x}_{\boldsymbol{n}} \cap \boldsymbol{N}\left(\boldsymbol{x}_{\boldsymbol{n}}\right) .
$$

Pela construção, é claro que $\boldsymbol{x}_{\mathbf{0}} \supseteq \boldsymbol{x}_{\mathbf{1}} \supseteq \ldots$.. Vamos mostrar como construir $\boldsymbol{N}(\boldsymbol{x})$ garantindo que todas as caixas $\boldsymbol{x}_{\boldsymbol{n}}$ contém $S_{z}$. Essa construção não é única e estamos seguindo as idéias de Hansen e Sengupta (1981).

Pela relação 5.43, garantimos que $\boldsymbol{x}_{\boldsymbol{n}+\mathbf{1}} \supseteq S_{z}$, desde que $\boldsymbol{N}\left(\boldsymbol{x}_{\boldsymbol{n}}\right)$ seja solução de

$$
\boldsymbol{J}\left(\boldsymbol{x}_{\boldsymbol{n}}\right)(y-z)=-\boldsymbol{f}\left(\boldsymbol{z}^{\boldsymbol{I}}\right) .
$$

Esse sistema fica bem definido se determinarmos $z$. Como temos liberdade na escolha de $z$, vamos tomá-lo como sendo uma aproximação do ponto médio da caixa $\boldsymbol{x}_{\boldsymbol{n}}$.

Nosso objetivo é encontrar uma caixa limitante de (5.46) em $\boldsymbol{x}_{\mathbf{0}}$. Aplicando o procedimento de pré-condicionamento descrito na seção 2.3 temos

$$
\boldsymbol{B}\left(y-\operatorname{mid}\left(\boldsymbol{x}_{\mathbf{0}}\right)\right)=\boldsymbol{b}
$$

onde $\boldsymbol{B}=\operatorname{mid}\left(\boldsymbol{J}\left(\boldsymbol{x}_{\mathbf{0}}\right)\right)^{-1} \boldsymbol{J}\left(\boldsymbol{x}_{\mathbf{0}}\right), \boldsymbol{b}=-\operatorname{mid}\left(\boldsymbol{J}\left(\boldsymbol{x}_{\mathbf{0}}\right)\right)^{-1} \boldsymbol{f}\left(\boldsymbol{x}^{I}\right)$ e $y \in \boldsymbol{x}_{\mathbf{0}}$.

Para resolver (5.47) utilizamos a versão intervalar do método de Gauss-Seidel, descrita no capítulo 2. O Algoritmo Newton Intervalar, que apresentamos no apêndice A, recebe a extensão de uma função $\boldsymbol{f}$ e sua matriz jacobiana $\boldsymbol{J}$. Ele devolve um conjunto de caixas que contém todas as raízes da função $f: \mathbb{R}^{n} \rightarrow \mathbb{R}^{n}$ em $\boldsymbol{x}_{\mathbf{0}}$.

A estratégia padrão em otimização global para resolver (5.1)-(5.5) é construir o sistema KKT do problema, limitar os multiplicadores de Lagrange e buscar todas as raízes do sistema com o método de Newton intervalar. Para nós, a abordagem padrão tem duas desvantagens. A primeira é que ela dobra o número de variáveis de decisão. A segunda é que perdemos a estrutura linear das restrições e passamos a nos preocupar com um sistema não linear. 
Em nossa implementação, seguimos um caminho diferente. Considere o $M M O_{2,2}$ e a estimação de seus parâmetros, dada por

$$
\begin{aligned}
\max z & =L(\theta) \\
a_{11}+a_{12} & =1 \\
a_{21}+a_{22} & =1 \\
b_{11}+b_{12} & =1 \\
b_{21}+b_{22} & =1 \\
\pi_{1}+\pi_{2} & =1 \\
a_{i j}, b_{i j}, \pi_{i} & \geq 0 .
\end{aligned}
$$

Esse problema é equivalente a

$$
\begin{array}{r}
\max z=L(\bar{\theta}+Z \gamma) \\
0 \leq a_{11}, a_{21}, b_{11}, b_{21}, \pi_{1} \leq 1,
\end{array}
$$

onde

$$
\gamma=\left(\begin{array}{c}
a_{12} \\
a_{22} \\
b_{12} \\
b_{22} \\
\pi_{2}
\end{array}\right), \quad Z=\left(\begin{array}{ccccc}
-1 & 0 & 0 & 0 & 0 \\
1 & 0 & 0 & 0 & 0 \\
0 & -1 & 0 & 0 & 0 \\
0 & 1 & 0 & 0 & 0 \\
0 & 0 & -1 & 0 & 0 \\
0 & 0 & 1 & 0 & 0 \\
0 & 0 & 0 & -1 & 0 \\
0 & 0 & 0 & 1 & 0 \\
0 & 0 & 0 & 0 & -1 \\
0 & 0 & 0 & 0 & 1
\end{array}\right) \quad \bar{\theta}=\left(\begin{array}{l}
1 \\
0 \\
1 \\
0 \\
1 \\
0 \\
1 \\
0 \\
1 \\
0
\end{array}\right) .
$$

É claro que podemos estender essa formulação para problemas com mais parâmetros. Essa construção é interessante pois reduz o problema original a um com restrições de caixas. Além disso, diminuímos o número de variáveis de decisão, sem aumentar a região de busca. Novamente, a estratégia padrão para resolver esse problema é aplicar o algoritmo de Newton ao vetor $\nabla L$. Devemos tomar cuidado ao aplicar esta estratégia pois é possível que pontos na borda da caixa sejam máximos globais e não satisfaçam $Z^{\prime} \nabla L(\bar{\theta}+Z \gamma)=0$. Dessa forma descartaríamos pontos indevidos.

O que fazemos é aplicar o método de Newton intervalar às caixas que não contém 0 ou $1 \mathrm{em}$ nenhuma coordenada. Nesses casos, buscamos as raízes do sistema $Z^{\prime} \nabla L(\bar{\theta}+Z \gamma)$, na caixa $\gamma$, obtida como no exemplo acima. O algoritmo é aplicado enquanto houver decréscimo de pelo menos $1 \%$ no maior diâmetro da caixa resultante. Caso o método de Newton não melhore a caixa, voltamos ao interval branch and bound.

\subsubsection{Propagação da Função Objetivo}

Seja $\boldsymbol{\theta}$ a caixa que estamos processando e $\theta^{*}$ um modelo viável, não necessariamente em $\boldsymbol{\theta}$. Considere ainda que $L\left(\theta^{*}\right)=L^{*}$. A propagação da função objetivo consiste em adicionar a seguinte restrição ao problema:

$$
L(\theta) \geq L\left(\theta^{*}\right) .
$$

O teorema de Taylor garante que se $\theta=\theta^{*}+h$ então

$$
L(\theta)=L\left(\theta^{*}\right)+\nabla L(\xi)^{\prime}\left(\theta-\theta^{*}\right)
$$

para algum $\xi \in \boldsymbol{\theta}$. Segue daí que

$$
L\left(\theta^{*}\right)+\nabla L(\xi)^{\prime}\left(\theta-\theta^{*}\right) \geq L^{*} .
$$


Como $\xi \in \boldsymbol{\theta}$, temos

$$
\nabla \boldsymbol{L}(\boldsymbol{\theta})^{\prime}\left(\boldsymbol{\theta}-\theta^{*}\right) \geq L^{*} .
$$

Lembrando que o modelo é parametrizado por $\theta=(A, B, \pi)$, escrevemos

$$
\theta_{1}=a_{11}, \theta_{2}=a_{12}, \ldots, \theta_{N^{2}}=a_{N N}
$$

Além disso,

$$
\theta_{N^{2}+1}=b_{11}, \theta_{N^{2}+2}=b_{12}, \ldots, \theta_{N^{2}+N M}=b_{N M} .
$$

Finalmente,

$$
\theta_{N^{2}+N M+1}=\pi_{1}, \ldots, \theta_{N^{2}+N M+N}=\pi_{N} .
$$

Com essa notação, a propagação da função objetivo nos dá,

$$
\boldsymbol{\theta}_{\boldsymbol{i}}{ }^{\prime} \geq \boldsymbol{\theta}_{\boldsymbol{i}} \cap\left(\theta_{i}^{*}+\frac{L^{*}-\sum_{\substack{j=1 \\ j \neq i}}^{N^{2}+N M+N} \frac{\partial \boldsymbol{L}(\boldsymbol{\theta})}{\partial \theta_{j}}\left(\boldsymbol{\theta}_{\boldsymbol{j}}-\theta_{j}^{*}\right)}{\frac{\partial \boldsymbol{L}(\boldsymbol{\theta})}{\partial \theta_{i}}}\right) .
$$

Segue daí que se não há intersecção entre os dois conjuntos do lado direito da desigualdade, não há pontos em $\boldsymbol{\theta}$ que satisfaçam $L(\theta)>L(\theta *)$. Portanto podemos excluir essa caixa da busca. Além disso se,

$$
\sup \left(\theta_{i}^{*}+\frac{L^{*}-\sum_{\substack{j=1 \\ j \neq i}}^{N^{2}+N M+N} \frac{\partial \boldsymbol{L}(\boldsymbol{\theta})}{\partial \theta_{j}}\left(\boldsymbol{\theta}_{\boldsymbol{j}}-\theta_{j}^{*}\right)}{\frac{\partial \boldsymbol{L}(\boldsymbol{\theta})}{\partial \theta_{i}}}\right)<\sup \left(\boldsymbol{\theta}_{\boldsymbol{i}}\right)
$$

então reduzimos $\theta^{*}$ na direção $i$.

\subsection{Experimentos Numéricos}

Nesta seção comparamos nossa implementação do interval branch and bound com o resolvedor de otimização global BARON, descrito em Sahinidis (2014). Esse é um software proprietário que disponibiliza uma versão reduzida para uso acadêmico sem custos. Para usar o BARON podemos escrever nossos problemas na linguagem GAMS ou em MATLAB. Os testes abaixo acessam o resolvedor através da última opção. A versão acadêmica é capaz de resolver problemas com até 10 variáveis, 30 restrições e 50 operações não lineares. Essa configuração nos restringe a resolver instâncias do $M M O_{2,2}$ de tamanho $T$, com $T=5, \ldots, 11$.

Fixado o tamanho da amostra $T$, temos $2^{T-1}$ problemas possíveis. Isso porque sempre podemos associar o valor 0 à primeira observação. Veja a seção 5.5. No entanto, a instância que corresponde a todas as observações iguais a 0 tem infinitas soluções Seja $\theta=(A, B, \pi)$ onde $b_{11}=1, b_{22}=0$ e $(A, \pi)$ é uma cadeia de Markov qualquer. Esse modelo é tal que $L(\theta)=1$ e estamos em um máximo global. Em nossos experimentos descartamos essa instância para todo $T$. No total, o teste envolve 2025 problemas.

Os testes envolvem duas implementações do interval branch and bound. A primeira usa exclusivamente o método de Baum-Welch como algoritmo local. Na segunda, adotamos a heurística mista, descrita na seção 5.3. Essa estratégia consiste em usar o método dos gradientes projetados espectrais para calibrar o ponto inicial do algoritmo de Baum-Welch.

Os experimentos foram realizados em um laptop com processador core-i7 e 16Gb de memória RAM. Utilizamos o compilador $i c c++14.0 .2$ e opções de compilação -O3, -frounding-math e NDEBUG. As tolerâncias foram fixadas em $\epsilon_{X}=\epsilon_{F}=10^{-4}$. O tempo máximo para solução de um problema é de 500 segundos. Esses parâmetros foram escolhidos para manter compatibilidade com o BARON. Veja os resultados abaixo e nossas conclusões a seguir.

Para cada $T$, apresentamos dois gráficos. O primeiro nos diz quantas instâncias foram resolvidas em até 500 segundos. O segundo é um gráfico de comparação de desempenho, comum em otimização. Ele deve ser lido da seguinte forma. O valor inicial é a porcentagem de problemas para os quais um 

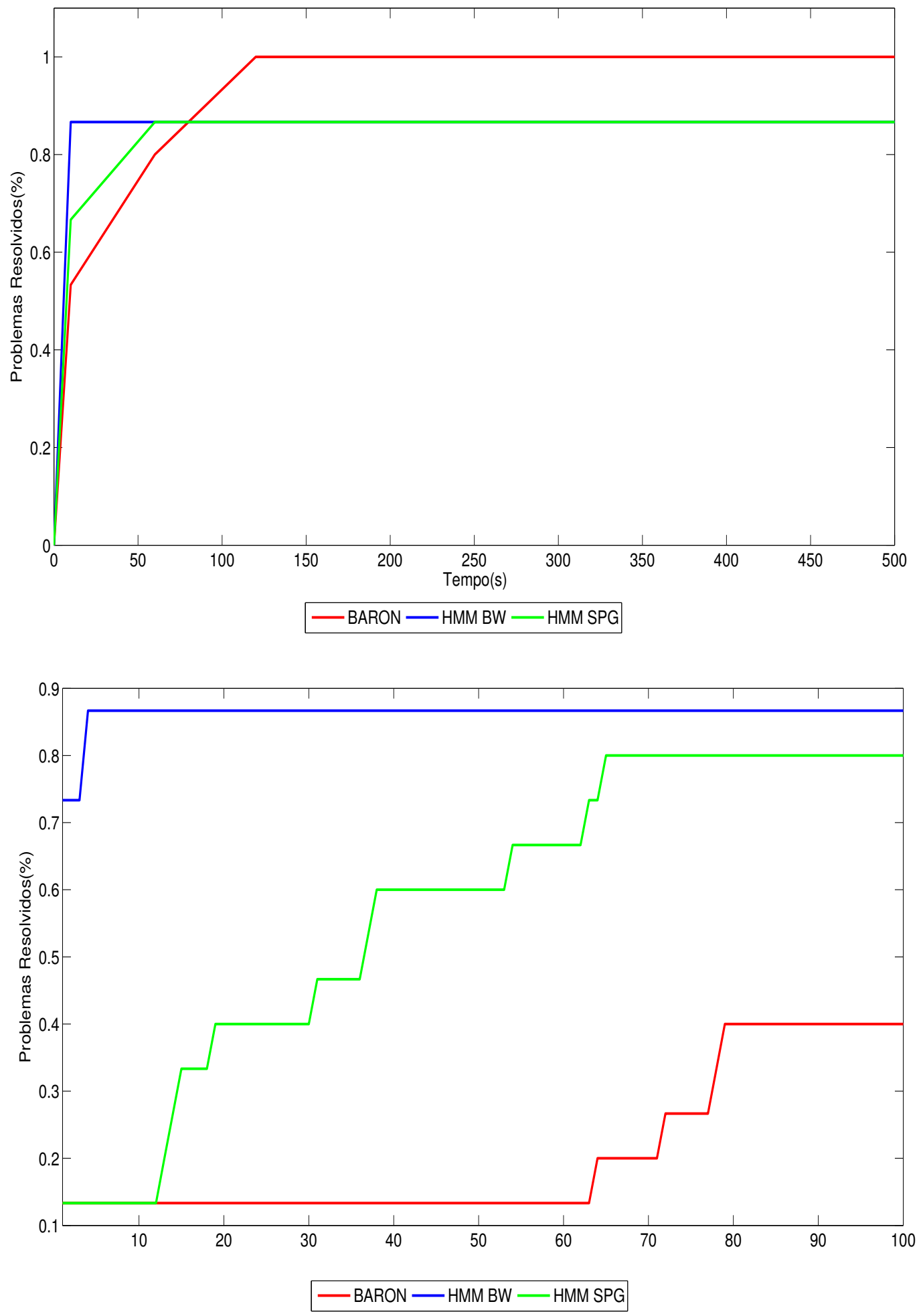

Figura 5.11: Comparação entre BARON, HMM SOLVER com Baum-Welch e HMM SOLVER com SPG. Teste realizado com o $M M O_{2,2}$. Todas as instâncias com 5 observações. 

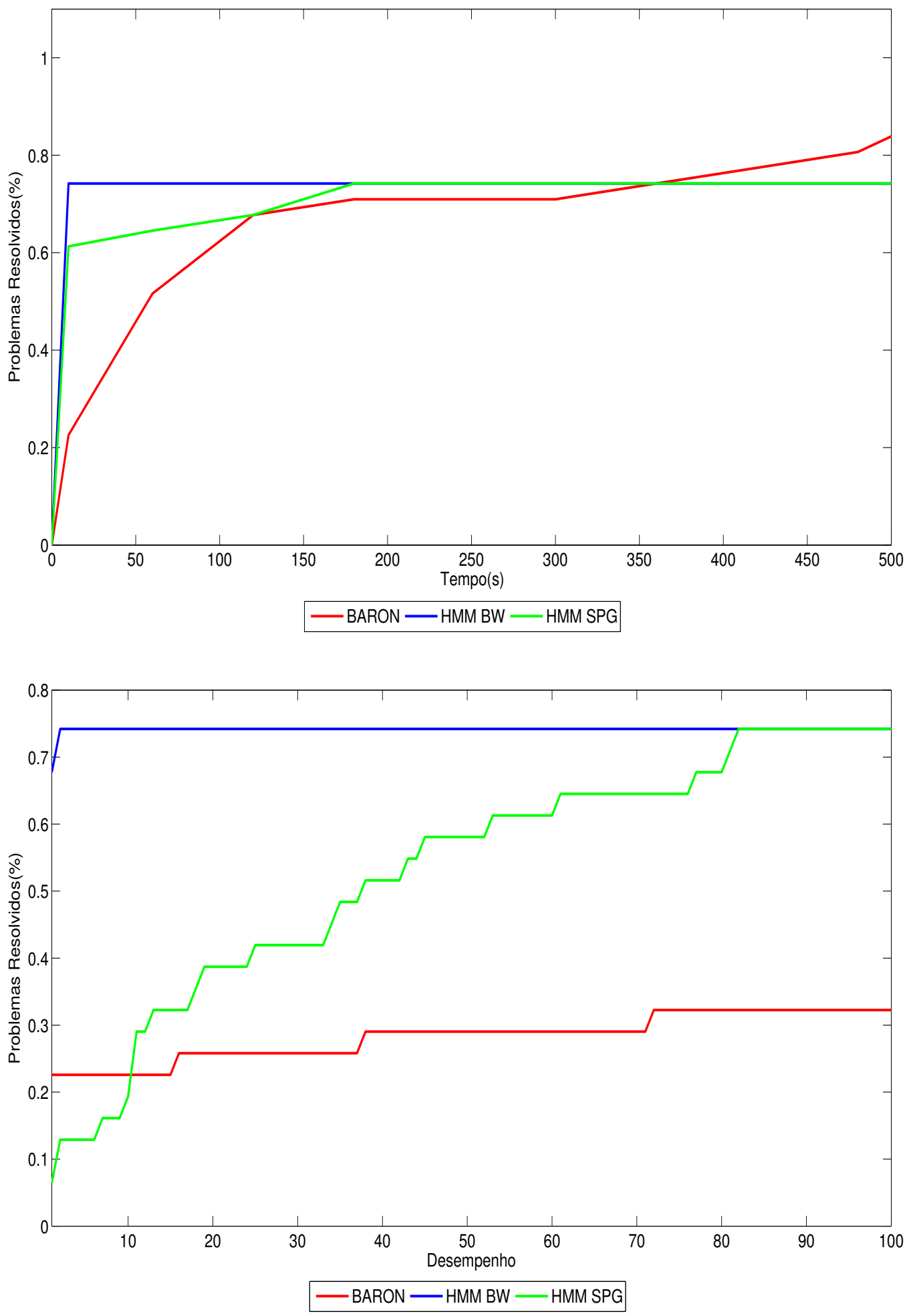

Figura 5.12: Comparação entre BARON, HMM SOLVER com Baum-Welch e HMM SOLVER com SPG. Teste realizado com o $M M O_{2,2}$. Todas as instâncias com 6 observações. 

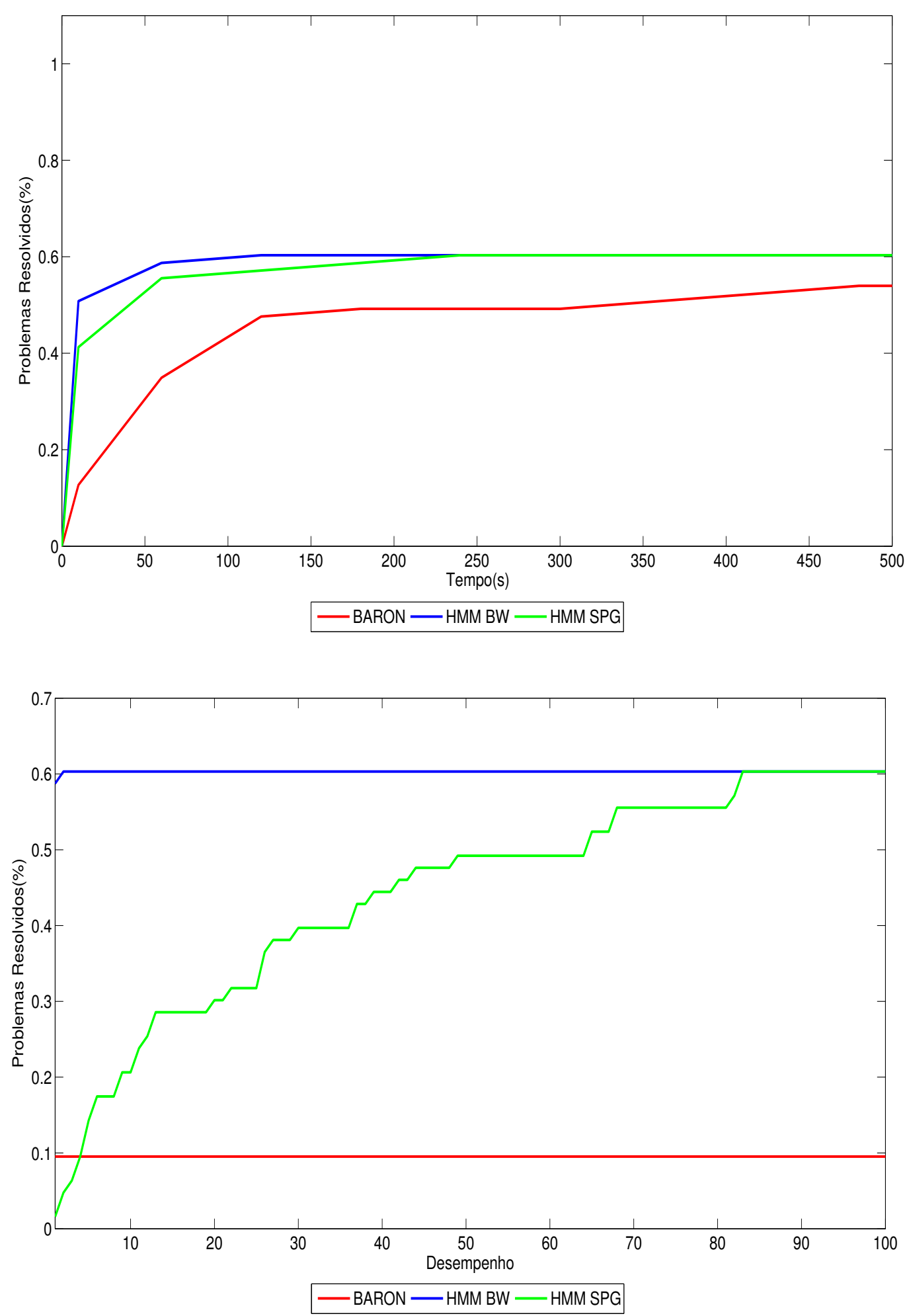

Figura 5.13: Comparação entre BARON, HMM SOLVER com Baum-Welch e HMM SOLVER com SPG. Teste realizado com o $\mathrm{MMO}_{2,2}$. Todas as instâncias com 7 observações. 

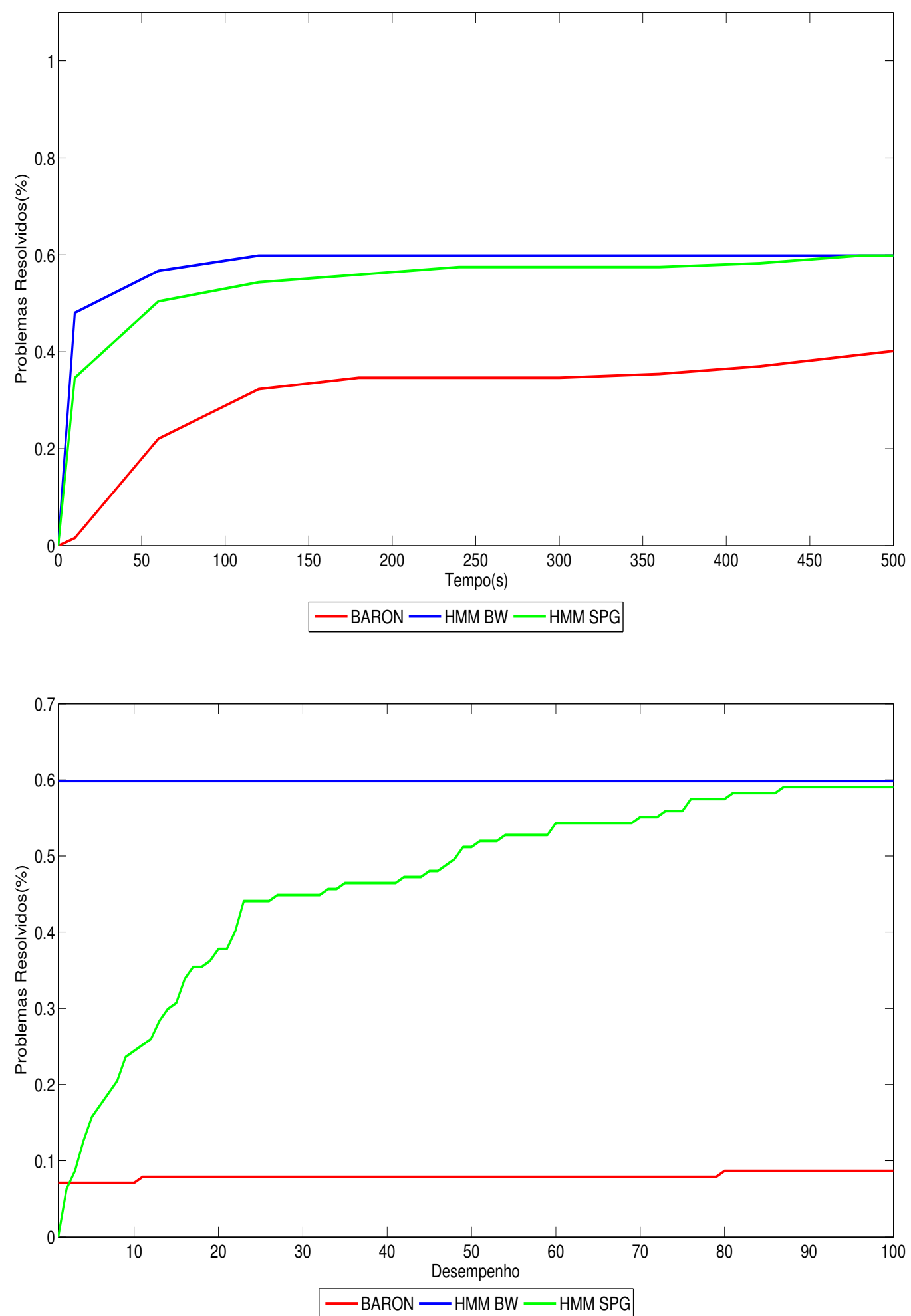

Figura 5.14: Comparação entre BARON, HMM SOLVER com Baum-Welch e HMM SOLVER com SPG. Teste realizado com o $M M O_{2,2}$. Todas as instâncias com 8 observações. 

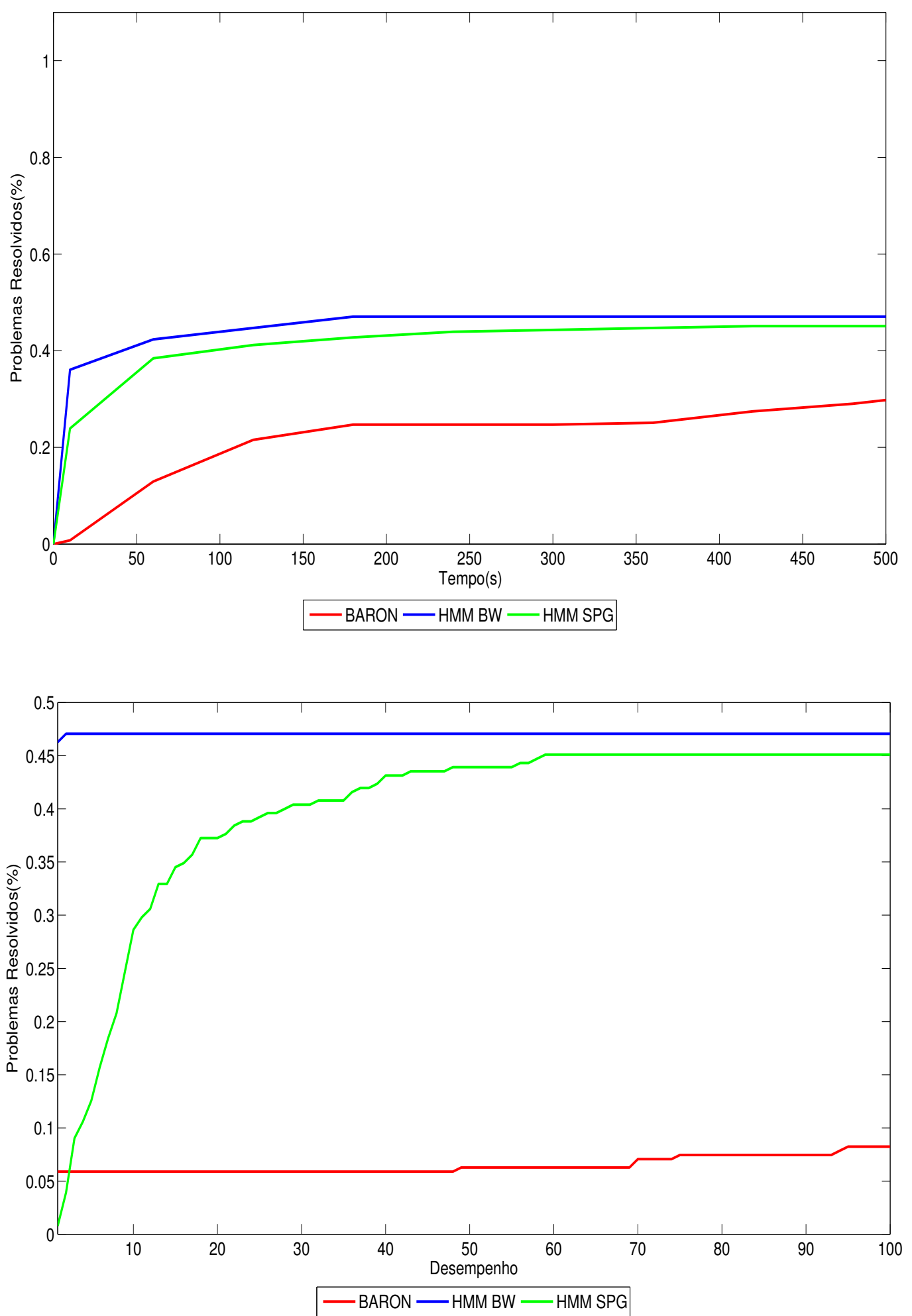

Figura 5.15: Comparação entre BARON, HMM SOLVER com Baum-Welch e HMM SOLVER com SPG. Teste realizado com o $M M_{2,2}$. Todas as instâncias com 9 observações. 

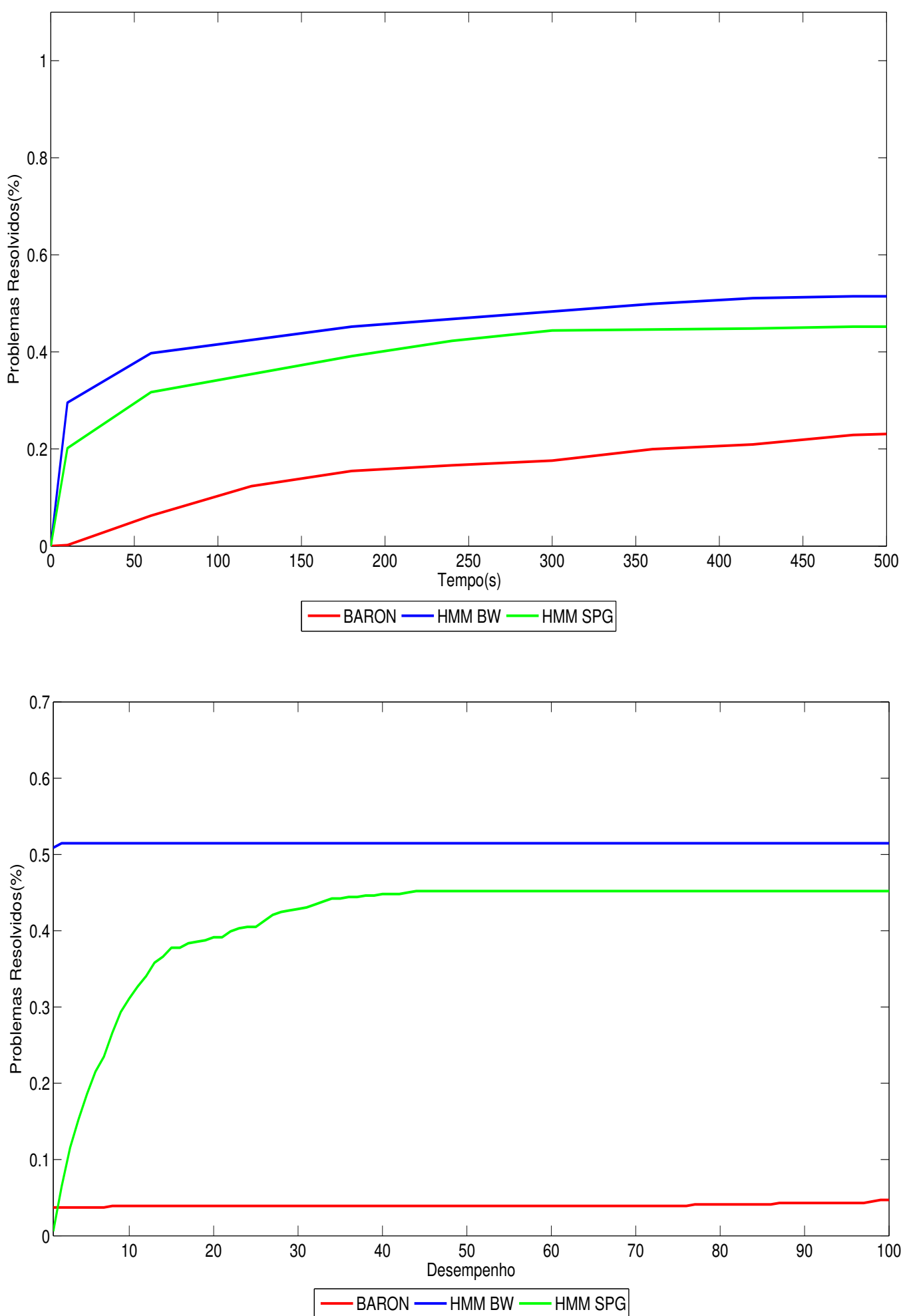

Figura 5.16: Comparação entre BARON, HMM SOLVER com Baum-Welch e HMM SOLVER com SPG. Teste realizado com o $M M_{2,2}$. Todas as instâncias com 10 observações. 

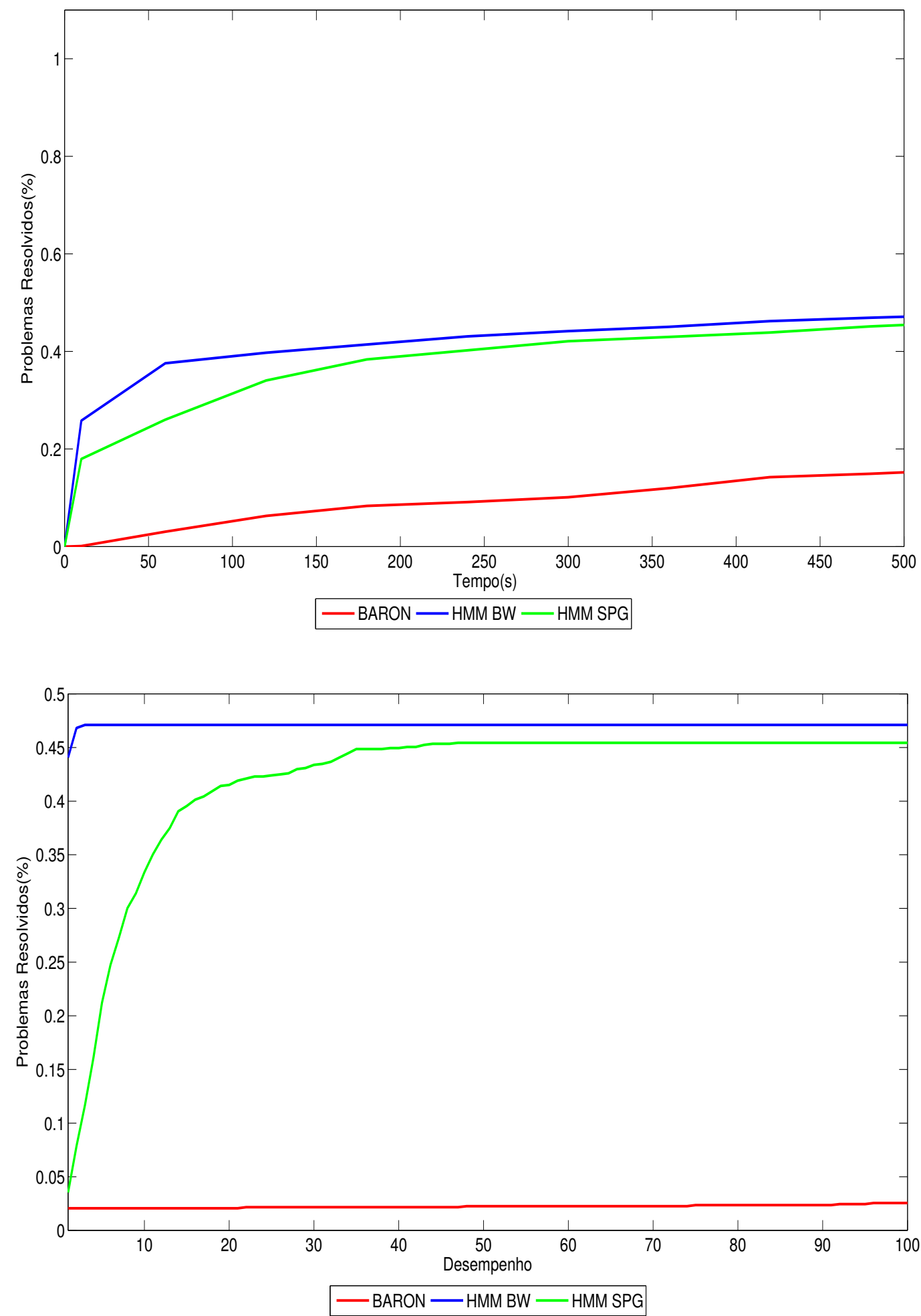

Figura 5.17: Comparação entre BARON, HMM SOLVER com Baum-Welch e HMM SOLVER com SPG. Teste realizado com o $\mathrm{MMO}_{2,2}$. Todas as instâncias com 11 observações. 

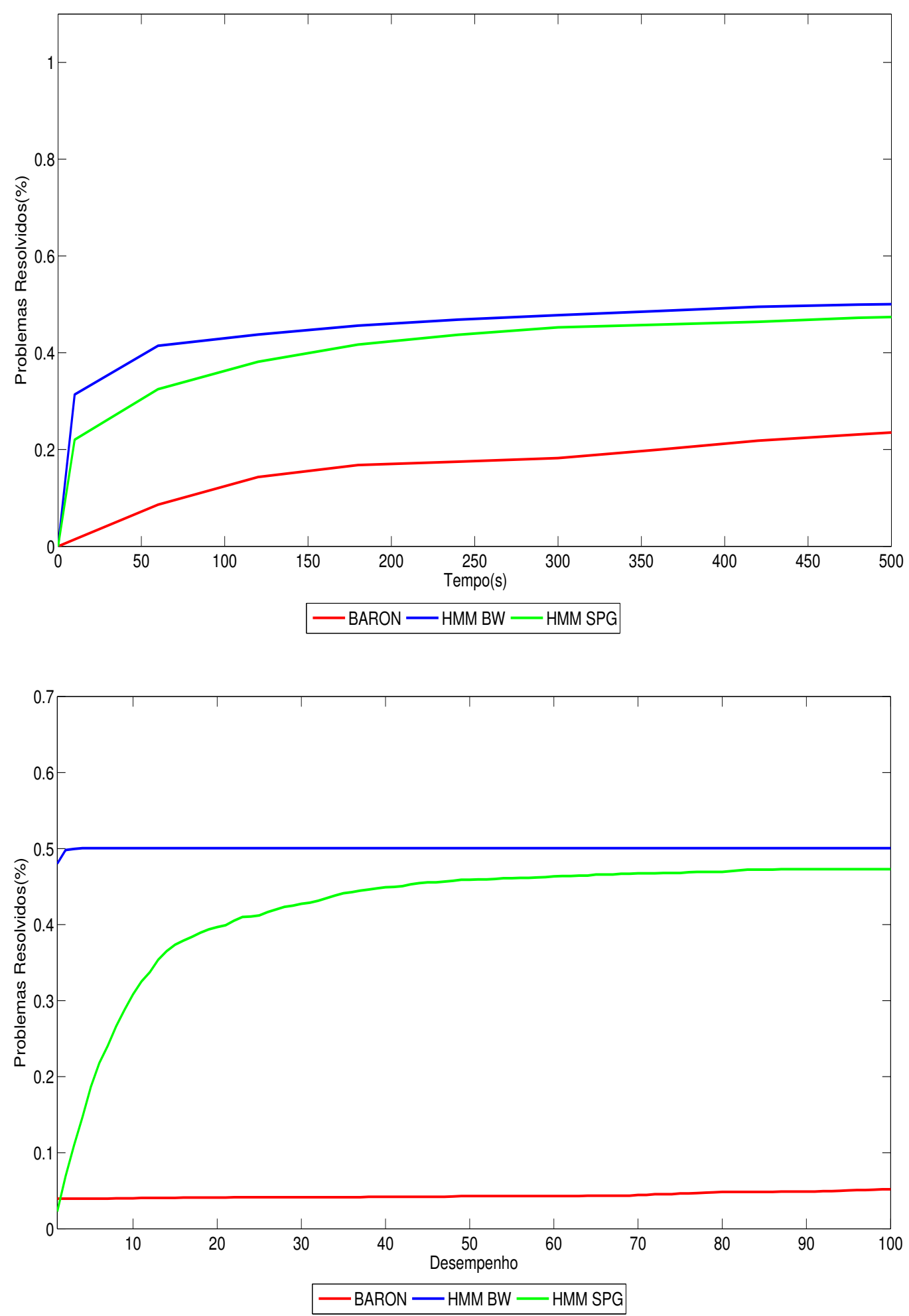

Figura 5.18: Comparação entre BARON, HMM SOLVER com Baum-Welch e HMM SOLVER com SPG. Teste realizado em modelo de Markov oculto com mistura Bernoulli. Todas as instâncias testadas. 
dado método é o mais eficiente. Em seguida, toda vez que a linha desse método dá um salto, temos o custo computacional necessário para resolver um problema a mais.

Por exemplo, suponha que o HMM SPG seja o melhor dos três na solução de $20 \%$ dos problemas. Então a linha desse método começa em 0.2. Suponha agora que o primeiro salto desse método é na abscissa 10. Isso quer dizer que, para resolver um problema a mais, o HMM SPG foi 10 vezes mais lento do que o melhor método naquela instância resolvida.

Dos 2025 problemas propostos, fomos capazes de resolver 1013 instâncias com o $H M M B W$ e 959 com o $H M M S P G$ contra as 476 instâncias resolvidas pelo $B A R O N$. Isto é, resolvemos por volta de $50 \%$ mais problemas do que o BARON nos primeiros 500 segundos de execução. Além disso, os gráficos de desempenho mostram que, para os problemas resolvidos, o $B A R O N$ precisa de mais do que 100 vezes o tempo da melhor implementação para resolver o mesmo problema. Mostramos ainda que a melhor heurística de busca para problemas pequenos é usar unicamente o Baum-Welch como algoritmo local.

A tabela abaixo apresenta o valor da verossimilhança na solução global obtida com cada método. Das 2025 instâncias testadas apresentamos aqui as 25 com maior norma da diferença entre os métodos. Fica claro pela tabela que os métodos são compatíveis com relação à precisão. Tanto

\begin{tabular}{|c|c|c|c|}
\hline$f^{*}$ BARON & $f^{*}$ HMM BW & $f^{*}$ HMM SPG & NORMA \\
\hline 0.005679 & 0.006066 & 0.000192 & 0.001158 \\
\hline 0.003517 & 0.003959 & 0.000121 & 0.001126 \\
\hline 0.005487 & 0.005847 & 0.000164 & 0.001048 \\
\hline 0.005323 & 0.00584 & 0.00586 & 0.001034 \\
\hline 0.003535 & 0.003869 & 0.000144 & 0.000956 \\
\hline 0.003474 & 0.003866 & 0.000083 & 0.00095 \\
\hline 0.005505 & 0.00596 & 0.000018 & 0.000946 \\
\hline 0.002507 & 0.002845 & 0.000128 & 0.000932 \\
\hline 0.00363 & 0.003858 & 0.000234 & 0.0009249 \\
\hline 0.003429 & 0.003852 & 0.000038 & 0.000922 \\
\hline 0.005964 & 0.006293 & 0.000127 & 0.000912 \\
\hline 0.003235 & 0.002986 & 0.000454 & 0.000908 \\
\hline 0.002525 & 0.002831 & 0.000146 & 0.000904 \\
\hline 0.005323 & 0.005775 & 0.005775 & 0.000904 \\
\hline 0.003449 & 0.00384 & 0.000058 & 0.000898 \\
\hline 0.002496 & 0.002744 & 0.0002 & 0.000896 \\
\hline 0.003469 & 0.003832 & 0.000079 & 0.000883 \\
\hline 0.002522 & 0.002732 & 0.000225 & 0.000871 \\
\hline 0.002442 & 0.002722 & 0.000145 & 0.000851 \\
\hline 0.004008 & 0.004302 & 0.000126 & 0.00084 \\
\hline 0.002422 & 0.002733 & 0.000107 & 0.000837 \\
\hline 0.002468 & 0.002715 & 0.000171 & 0.000837 \\
\hline 0.002528 & 0.00271 & 0.000231 & 0.000826 \\
\hline 0.002797 & 0.003 & 0.000202 & 0.00081 \\
\hline & & &
\end{tabular}

Tabela 5.1: Limitante superior obtido com os resolvedores BARON, HMM SOLVER com Baum-Welch e HMM SOLVER com spg. Lista das 25 instâncias com maior soma da norma das diferenças dentre todas as 2025 testadas.

o $H M M$ com Baum-Welch quanto com $S P G$ nos dão resultados próximos daquele obtidos com BARON. Sendo assim concluímos que nossa implementação é equivalente ao BARON em termos de precisão e mais eficiente do que o software geral. 


\subsection{Experimentos Numéricos}

Nesta seção realizamos experimentos com o $M M O_{2,2}$. Nossa principal contribuição aqui é estudar a distribuição dos maximizadores globais nos casos com poucas amostras.

$\mathrm{O} M M O_{2,2}$ foi escolhido porque é fácil visualizar resultados nele. Observe que esses modelos são da forma

$$
\pi^{T}=\left(\begin{array}{ll}
\pi_{1} & \pi_{2}
\end{array}\right) \quad A=\left(\begin{array}{ll}
a_{11} & a_{12} \\
a_{21} & a_{22}
\end{array}\right) \quad B=\left(\begin{array}{ll}
b_{11} & b_{12} \\
b_{21} & b_{22}
\end{array}\right) .
$$

Assim, escrevemos

$$
\pi^{T}=\left(\pi_{1} \pi_{2}\right) \quad A=\left(\begin{array}{cc}
a_{11} & 1-a_{11} \\
1-a_{22} & a_{22}
\end{array}\right) \quad B=\left(\begin{array}{cc}
b_{11} & 1-b_{11} \\
1-b_{22} & b_{22}
\end{array}\right) .
$$

Essa representação permite construir gráficos com eixos $a_{11} \mathrm{x} a_{22}$ e $b_{11} \times b_{22}$.

As figuras abaixo foram obtidas com nossa implementação do interval branch and bound. Para cada $T$ apresentamos todos os máximos globais encontrados durante a execução do algoritmo global. O experimento foi realizado em um Intel Xeon com 32Gb de memória RAM. As tolerâncias foram fixadas em $\epsilon_{X}=\epsilon_{F}=10^{-4}$. O tempo máximo de execução é de uma hora.

Os dois primeiros gráficos mostram a dispersão dos máximos globais para cada $T$. O último gráfico em cada página nos dá quantidade de problemas resolvidos em função do tempo. 

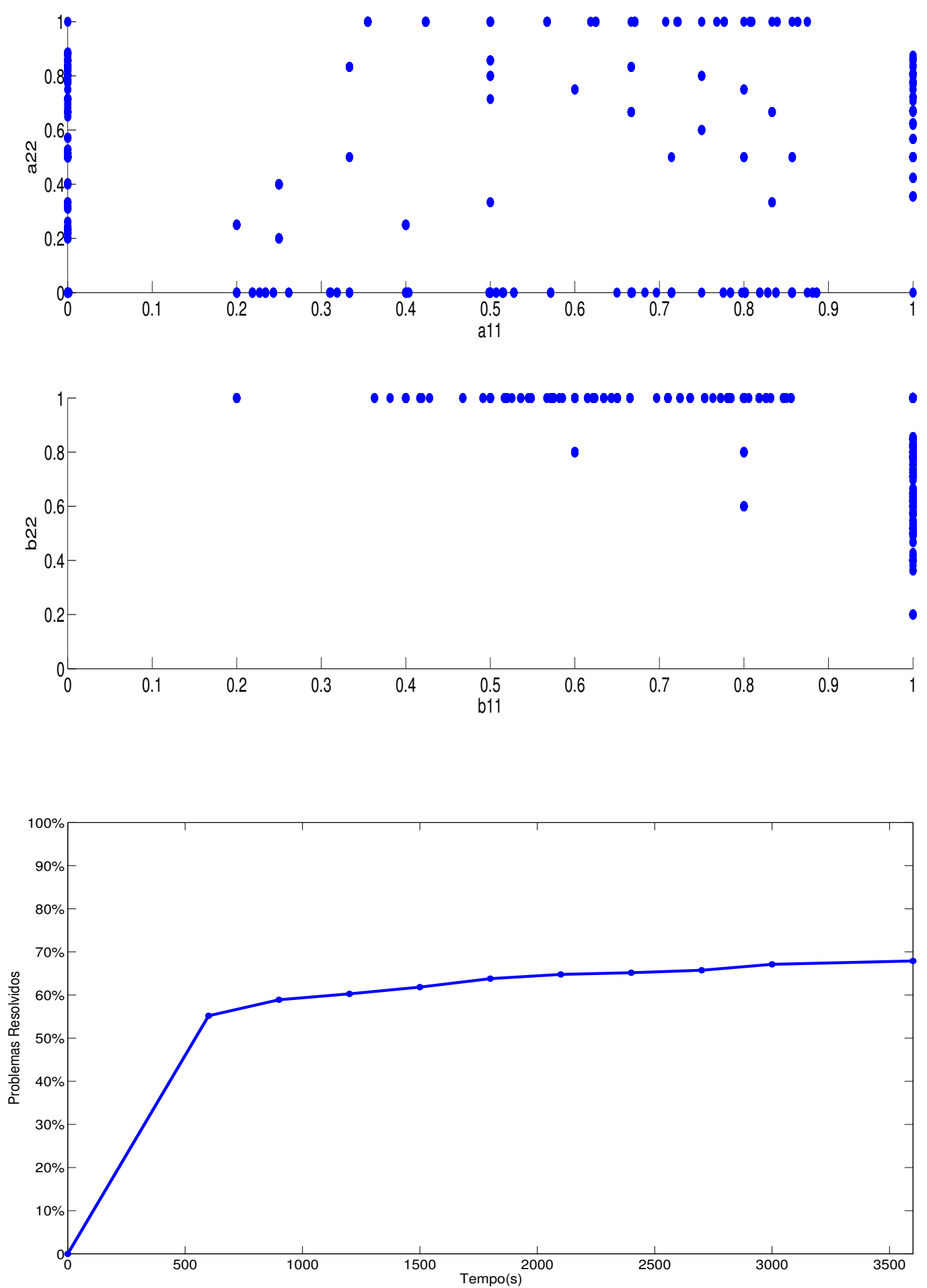

Figura 5.19: Dispersão dos máximos globais para o $M M_{2,2}$ com amostras de tamanho 10. 

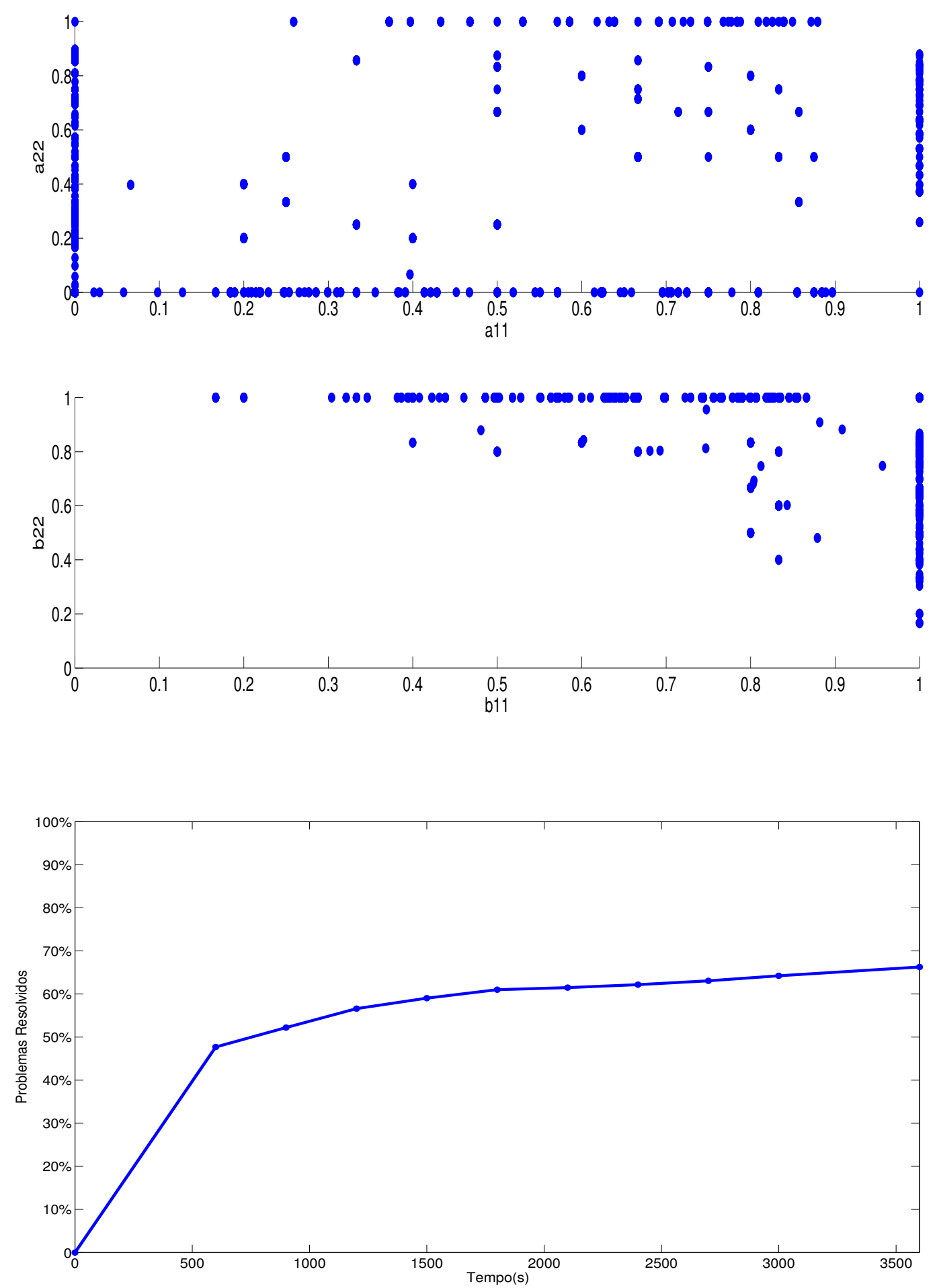

Figura 5.20: Dispersão dos máximos globais para o $M M_{2,2}$ com amostras de tamanho 11. 

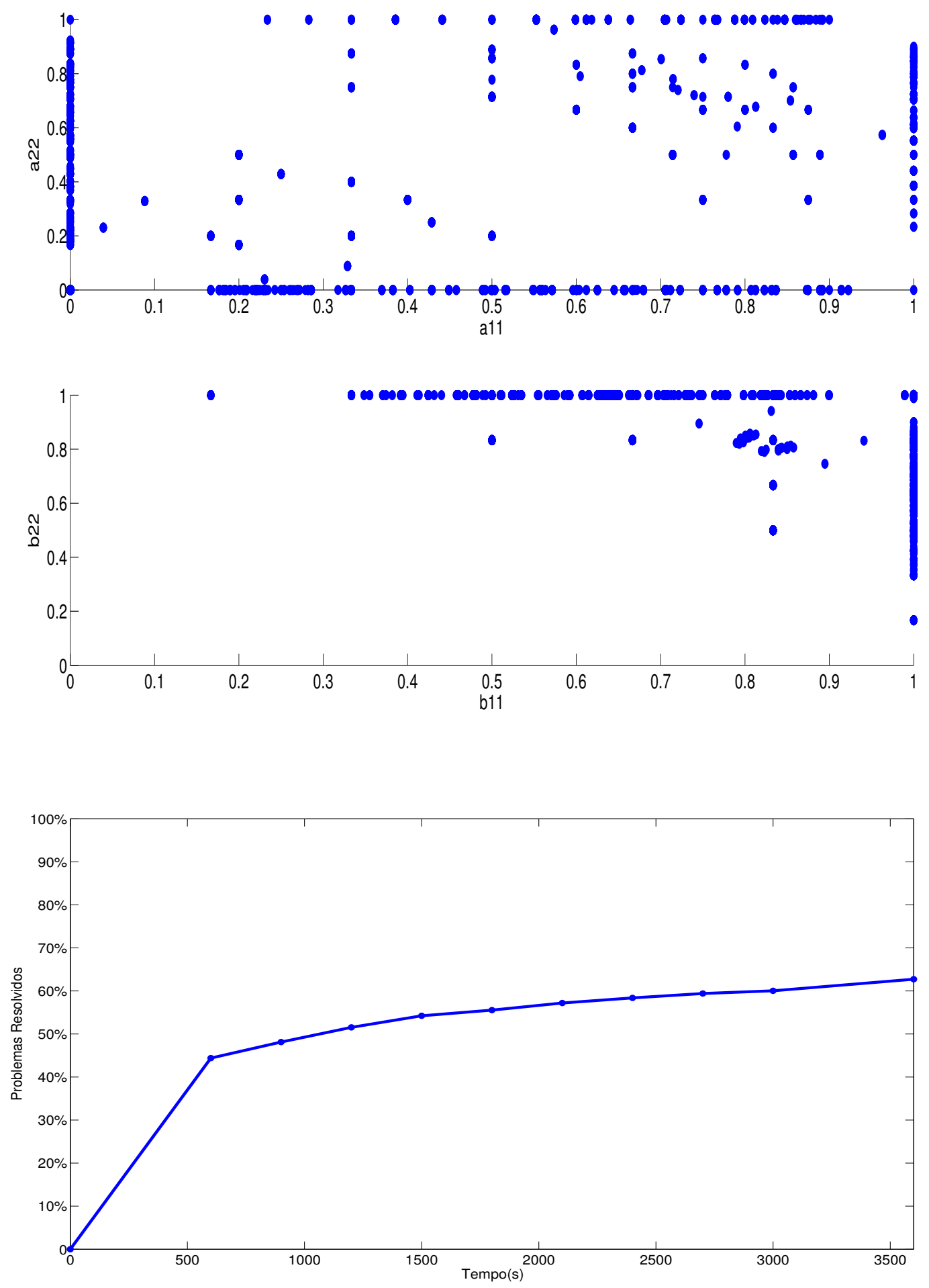

Figura 5.21: Dispersão dos máximos globais para o $M M_{2,2}$ com amostras de tamanho 12 . 

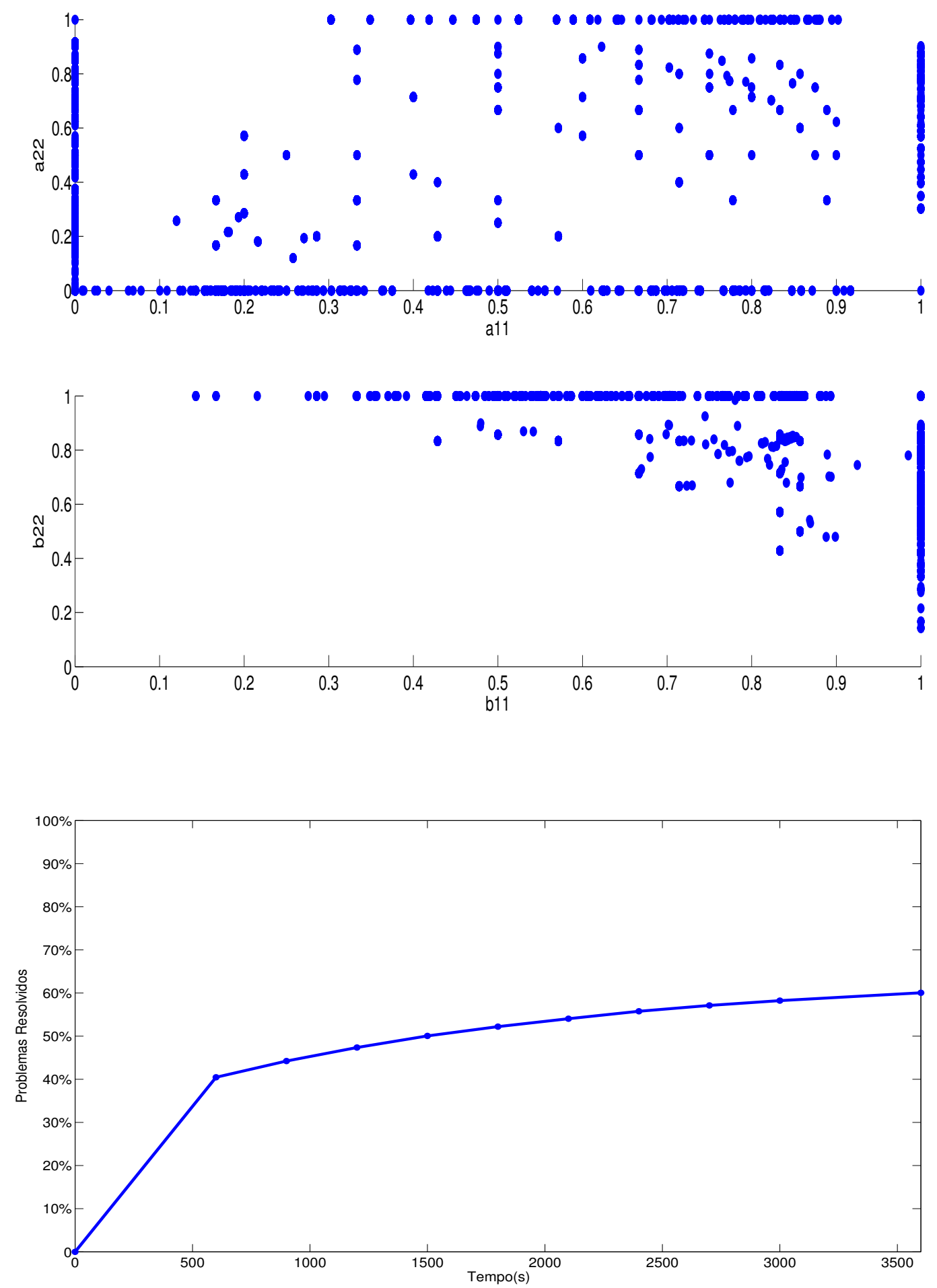

Figura 5.22: Dispersão dos máximos globais para o $M M_{2,2}$ com amostras de tamanho 13. 

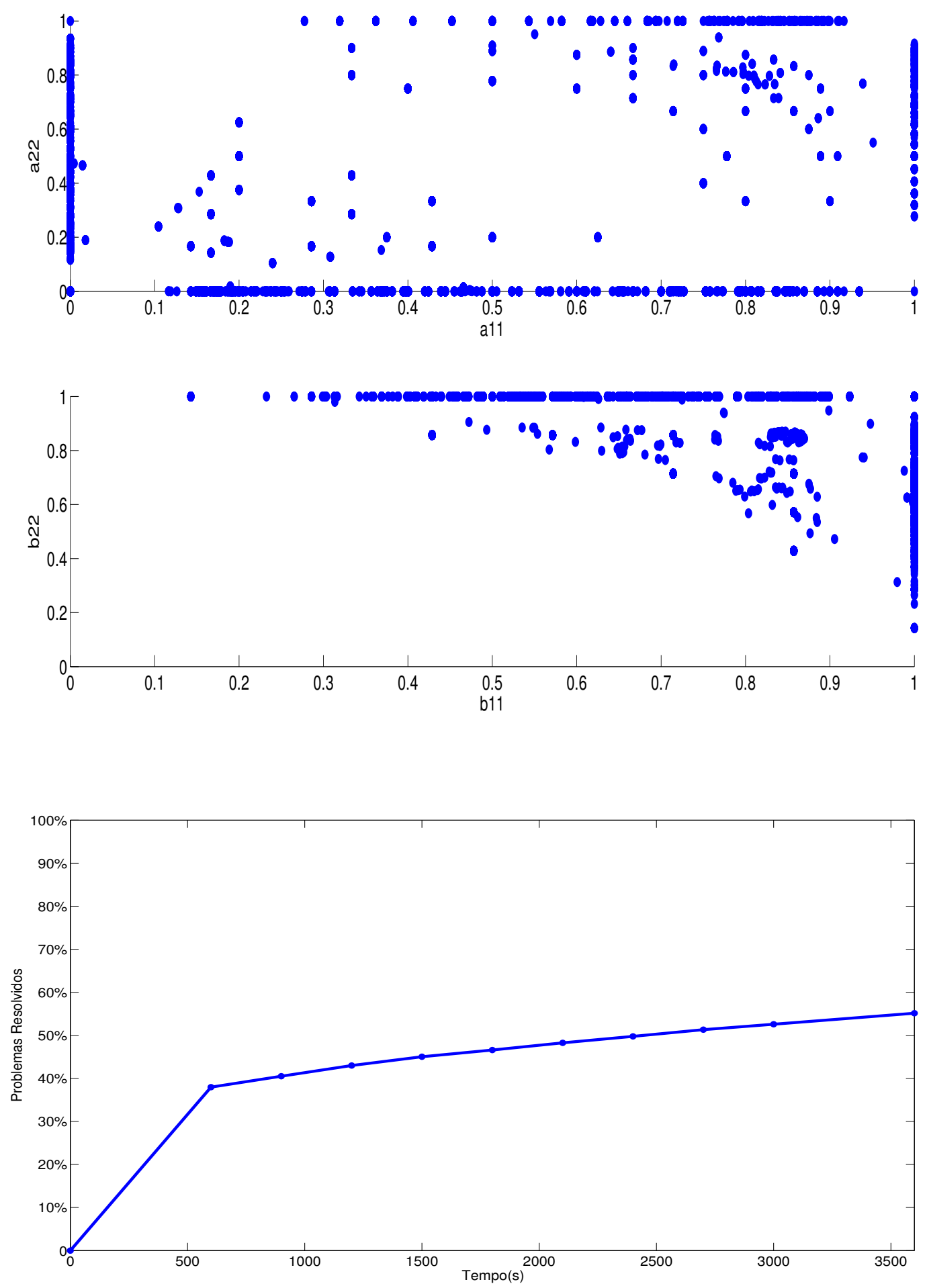

Figura 5.23: Dispersão dos máximos globais para o $M M_{2,2}$ com amostras de tamanho 14 . 

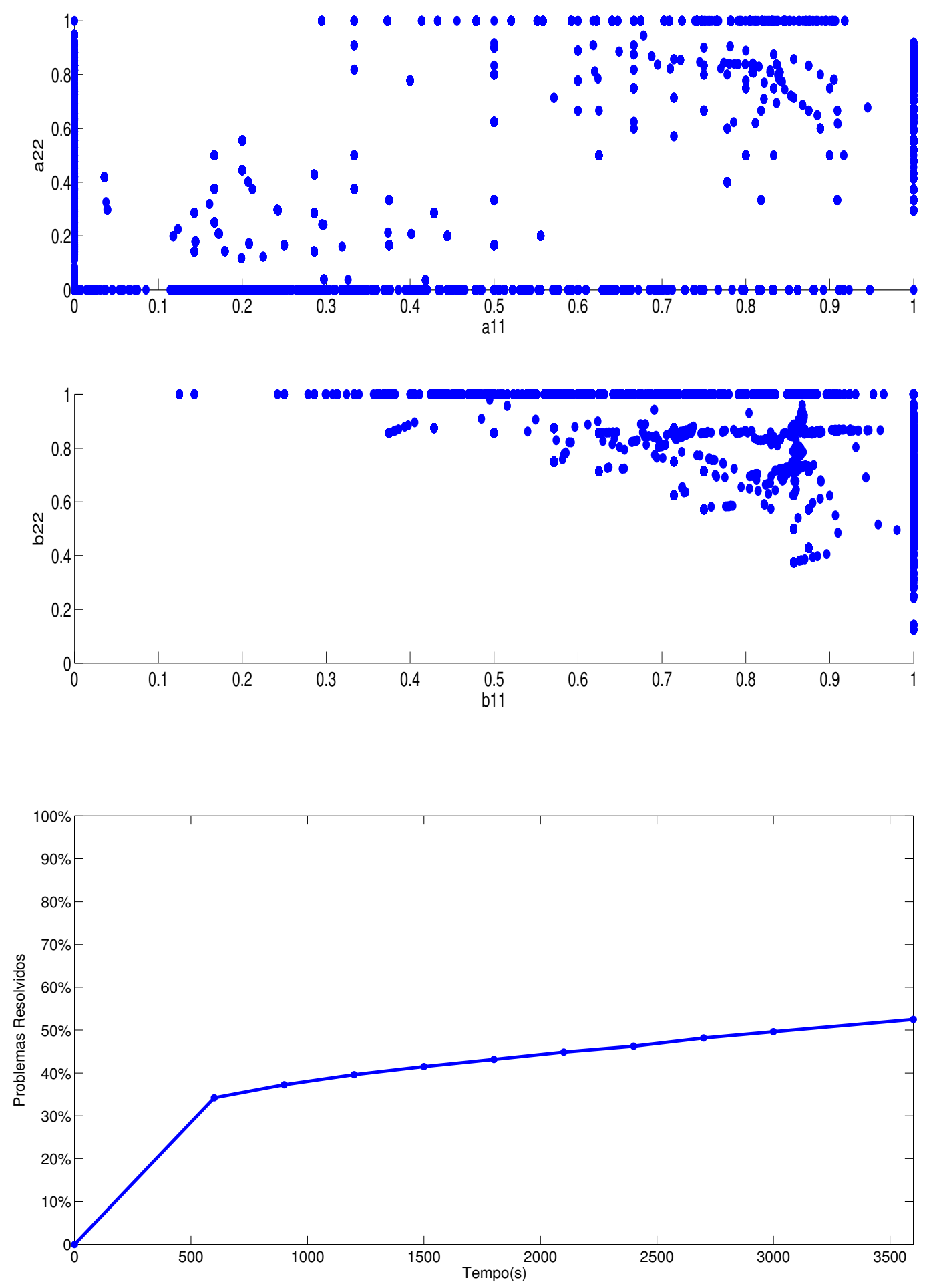

Figura 5.24: Dispersão dos máximos globais para o $M M_{2,2}$ com amostras de tamanho 15. 
Para $T>15$ menos da metade dos problemas foram resolvidos em até uma hora. Observamos um claro padrão em torno do eixo $y=-x$ para os gráficos da matriz de transição. Esse resultado precisa de mais experimentos com outra versão do nosso interval branch and bound para se confirmar. Notamos que os máximos globais estão bastante dispersos e modelos nas áreas brancas dos gráficos não seriam corretamente detectados. 


\section{Capítulo 6}

\section{Conclusões}

Utilizamos aritmética intervalar para construir um algoritmo de otimização global para estimar parâmetros em modelos de Markov ocultos. Nosso objetivo foi modificar o interval branch and bound para acelerar a convergência do método. Como resultado principal mostramos que nossa modificação é mais eficiente e tão precisa quanto o melhor resolvedor de otimização global disponível, o BARON.

Além disso, comparamos métodos locais de otimização. Nossa comparação levou em conta que esses algoritmos são parte integrante do procedimento global. Apresentamos o algoritmo de BaumWelch e os gradientes projetados espectrais. Esses métodos foram comparados ao de pontos interiores. Nossos experimentos indicam que o método de Baum-Welch é o mais indicado para MMOs com poucas observações. A escolha do algoritmo local não é imediata conforme aumentamos o tamanho da amostra.

Apresentamos uma nova extensão intervalar para o cálculo da verossimilhança. Nossa alternativa é mais eficiente e precisa do que a extensão natural. Ela utiliza apenas duas trocas no modo de arredondamento para calcular a função e suas derivadas. Os resultados mostram ganhos de 16 a 250 vezes no tempo e limitantes até 100 vezes mais estreitos.

Modificamos a normalização no cálculo da verossimilhança, substituindo o log por uma nova classe de números normalizados com funções ldexp e frexp. Nossa normalização dá os mesmos resultados daquela com $\log$ mas é cerca de 100 vezes mais eficiente.

Mostramos também como calcular a distribuição estacionária intervalar com base no algoritmo de Gauss-Seidel. Nossa alternativa é ligeiramente superior à função verifylss descrita em Rump (2001).

Mostramos ainda a distribuição dos máximos globais em $M M O_{2,2}$ com poucas observações. Tabelamos todos esses resultados e gostaríamos de utiliza-los na obtenção de novos limitantes superiores de $L(\theta)$.

De forma geral, acredito que atingimos alguns objetivos com a tese e nossa primeira versão do interval branch and bound modificado. No entanto, ainda há bastante trabalho para realizar nesses tópicos. Como próximos passos, destacamos,

1. Implementação de novas estratégias de aceleração da convergência, como a $\epsilon$-inflação, descrita em Kearfott (1996).

2. Paralelização do código.

3. Uso dos máximos globais já obtidos para poucas observações na obtenção de novos limitantes superiores de $L(\theta)$.

4. Resolver $100 \%$ das instâncias dos $M M O_{2,2}$ com poucas observações.

5. Implementar uma interface do nosso resolvedor com o $R$ e o $M A T L A B$. 
CONCLUSÕES 


\section{Apêndice A}

\section{Pseudo-Código dos Métodos Intervalares}

Apresentamos agora o pseudo-código dos métodos intervalares descritos ao longo da tese.

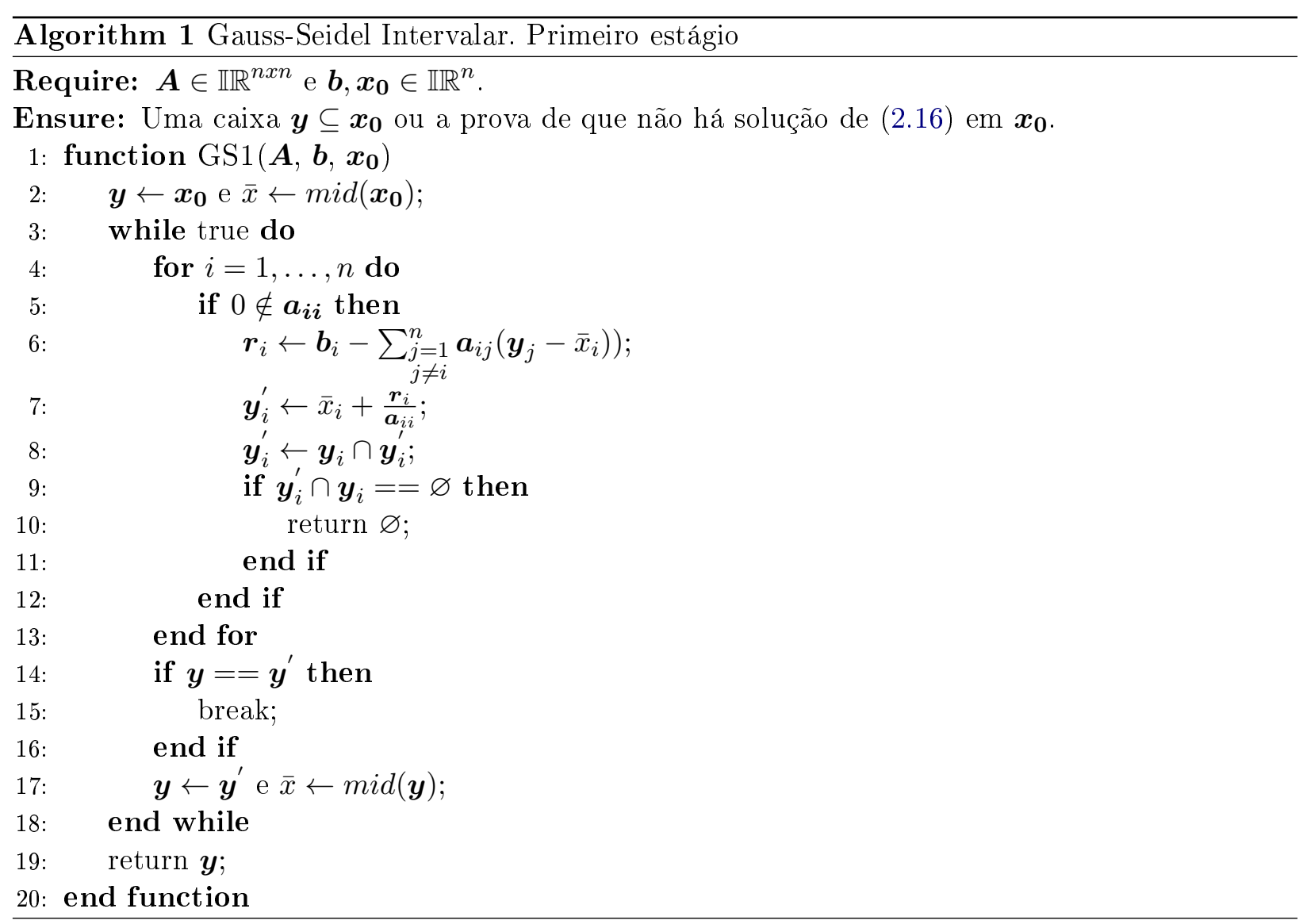




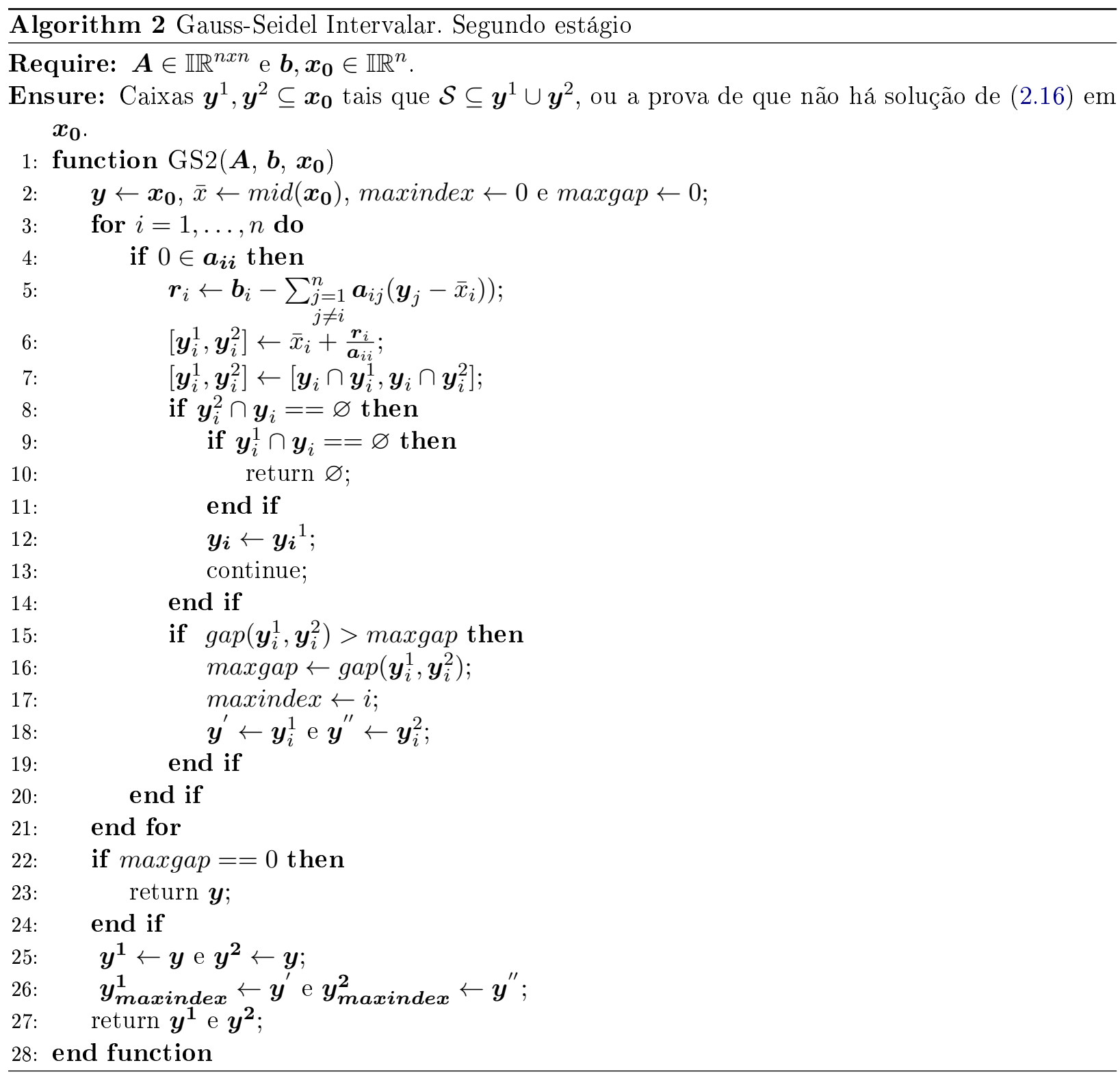




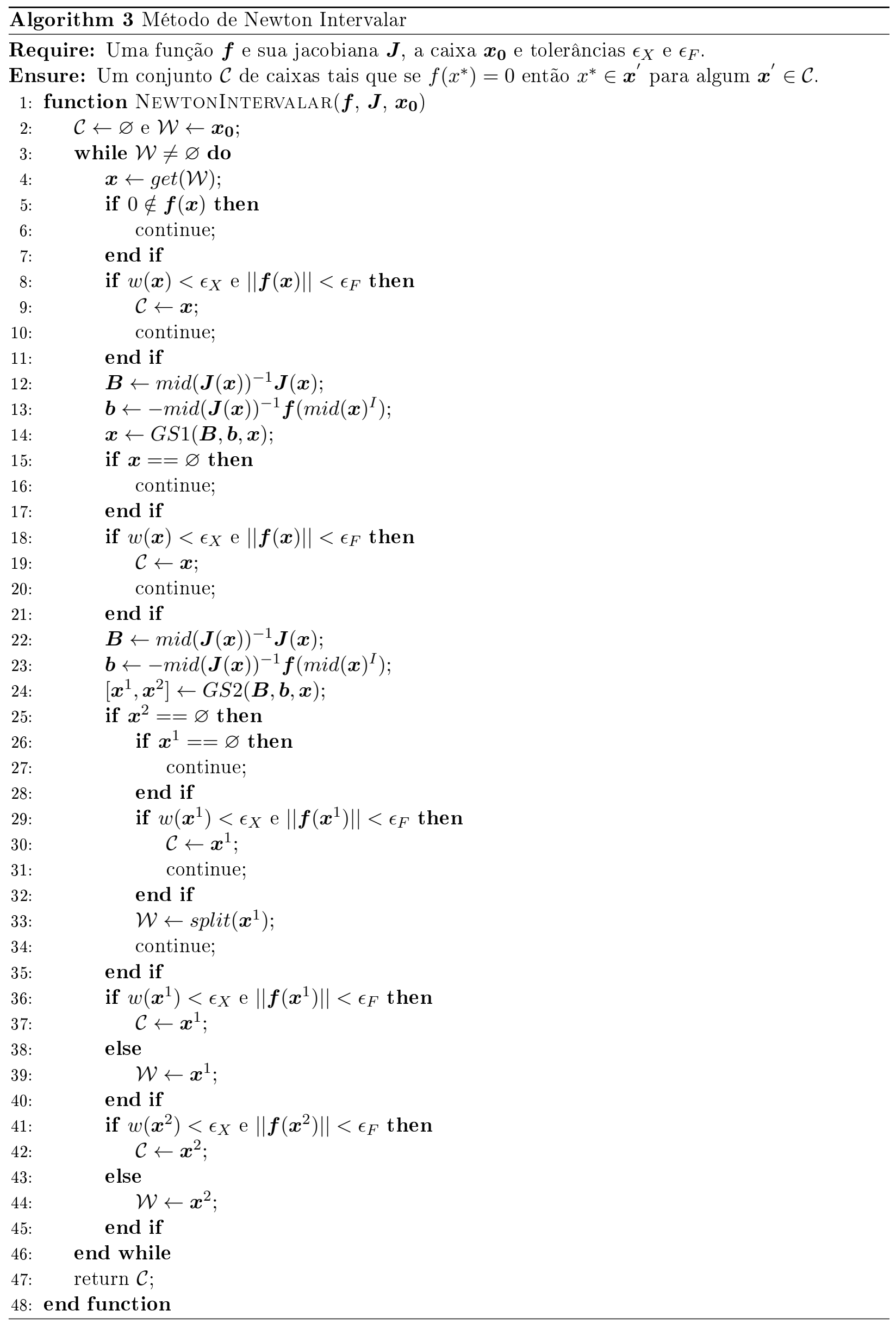




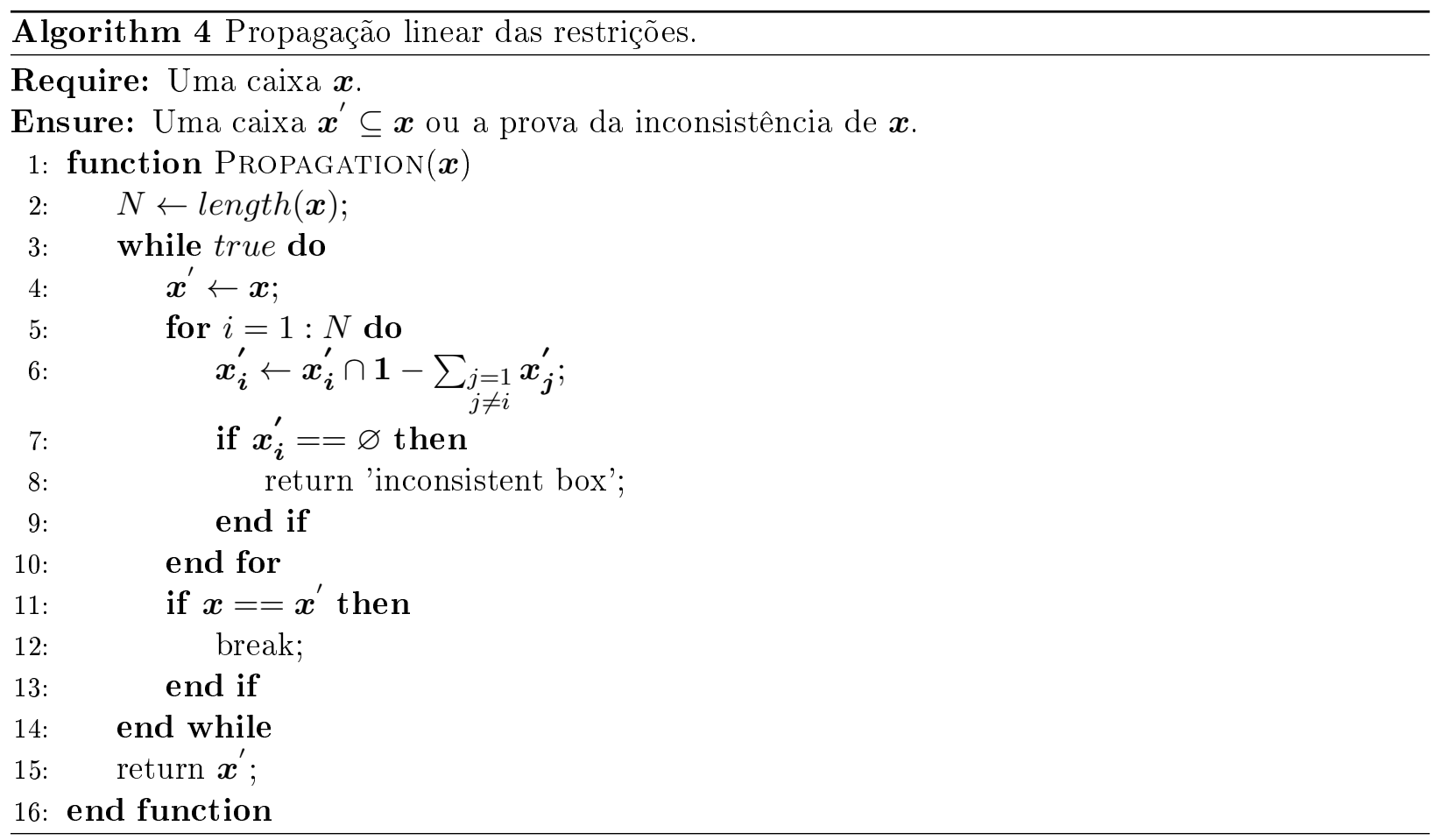




\section{Referências Bibliográficas}

754 (1985) IEEE 754. ANSI/IEEE 754-1985, Standard for Binary Floating-Point Arithmetic. IEEE, New York, 1985. Citado na pág. 6

Ahn et al. (2012) Hyo-Sung Ahn, Young-Soo Kim e Yangquan Chen. An interval Kalman filtering with minimal conservatism. Appl. Math. Comput., 218(18):9563-9570. ISSN 0096-3003. doi: 10.1016/j.amc.2012.02.050. Citado na pág. 4

Algahtani (2011) Obaid Jefain Algahtani. An Interval Kalman Filter, Interval Em Algorithm with Application to Weather Prediction. Tese de Doutorado. AAI3461791. Citado na pág. 4

Andreani et al. (2007) R. Andreani, E.G. Birgin, J.M. Martínez e M.L. Schuverdt. On augmented Lagrangian methods with general lower-level constraints. SIAM J. Optim., 18(4):1286-1309. ISSN 1052-6234; 1095-7189/e. doi: 10.1137/060654797. Citado na pág. 17

Andreani et al. (2008) R. Andreani, E.G. Birgin, J.M. Martínez e M.L. Schuverdt. Augmented Lagrangian methods under the constant positive linear dependence constraint qualification. Math. Program., 111(1-2 (B)):5-32. ISSN 0025-5610; 1436-4646/e. doi: 10.1007/s10107-006-0077-1. Citado na pág. 17

Aupetit et al. (2007) Sébastien Aupetit, Nicolas Monmarché e Mohamed Slimane. Hidden Markov models training by a particle swarm optimization algorithm. J. Math. Model. Algorithms, 6(2): 175-193. ISSN 1570-1166. doi: 10.1007/s10852-005-9037-7. URL http://dx.doi.org/10.1007/ s10852-005-9037-7. Citado na pág. 2

Barbu et al. (2012) Vlad Stefan Barbu, Jan Bulla e Antonello Maruotti. Estimation of the stationary distribution of a semi-Markov chain. J. Reliab. Stat. Stud., 5:15-26. ISSN 0974-8024; 2229-5666/e. Citado na pág. 2, 15

Baum e Eagon (1967) Leonard E. Baum e J. A. Eagon. An inequality with applications to statistical estimation for probabilistic functions of Markov processes and to a model for ecology. Bull. Amer. Math. Soc., 73:360-363. ISSN 0002-9904. Citado na pág. 2, 15

Baum e Petrie (1966) Leonard E. Baum e Ted Petrie. Statistical inference for probabilistic functions of finite state Markov chains. Ann. Math. Statist., 37:1554-1563. ISSN 0003-4851. Citado na pág. $2,15,24$

Baum et al. (1970) Leonard E. Baum, Ted Petrie, George Soules e Norman Weiss. A maximization technique occurring in the statistical analysis of probabilistic functions of Markov chains. Ann. Math. Statist., 41:164-171. ISSN 0003-4851. Citado na pág. 47

Bhar e Hamori (2004) Ram Bhar e Shigeyuki Hamori. Hidden Markov models : applications to financial economics. Kluwer Academic Publishers Boston, Mass. ISBN 1402078994. Citado na pág. 2

Birgin et al. (2000) Ernesto G. Birgin, Jos É Mario Martínez e Marcos Raydan. Nonmonotone spectral projected gradient methods on convex sets. SIAM Journal on Optimization, páginas 1196-1211. Citado na pág. 47, 48, 50 
Bulla et al. (2010) Jan Bulla, Ingo Bulla e Oleg Nenadi? hsmm : An r package for analyzing hidden semi-markov models. Computational Statistics and Data Analysis, 54(3):611 - 619. ISSN 0167-9473. Second Special Issue on Statistical Algorithms and Software. Citado na pág. 2, 15, 25

Burge e Karlin (1997) Chris Burge e Samuel Karlin. Prediction of complete gene structures in human genomic \{DNA\}. Journal of Molecular Biology, 268(1):78 - 94. ISSN 0022-2836. Citado na pág. 2

Calamai e Moré (1987) Paul H. Calamai e Jorge J. Moré. Projected gradient methods for linearly constrained problems. Math. Program., 39:93-116. ISSN 0025-5610; 1436-4646/e. doi: 10.1007/BF02592073. Citado na pág. 48

Cappe e Moulines (2005) O. Cappe e E. Moulines. Recursive computation of the score and observed information matrix in hidden markov models. Em Statistical Signal Processing, 2005 IEEE/SP 13th Workshop on, páginas 703-708. Citado na pág. 2

Cappé et al. (2005) Olivier Cappé, Eric Moulines e Tobias Ryden. Inference in Hidden Markov Models (Springer Series in Statistics). Springer-Verlag New York, Inc., Secaucus, NJ, USA. ISBN 0387402640. Citado na pág. 2, 15, 25

Csendes (2004) Tibor Csendes. Generalized subinterval selection criteria for interval global optimization. Numer. Algorithms, 37(1-4):93-100. ISSN 1017-1398. doi: 10.1023/B:NUMA. 0000049489.44154.02. URL http://dx.doi.org/10.1023/B:NUMA.0000049489.44154.02. Citado na pág. 5

Dempster et al. (1977) A. P. Dempster, N. M. Laird e D. B. Rubin. Maximum likelihood from incomplete data via the em algorithm. JOURNAL OF THE ROYAL STATISTICAL SOCIETY, SERIES B, 39(1):1-38. Citado na pág. 17, 47

Feller (1968) William Feller. An Introduction to Probability Theory and Its Applications, volume 1. Wiley. Citado na pág. 15,18

Floudas (2000) C. A. Floudas. Deterministic Global Optimization. Kluwer Academic, Dordrecht, first edition ed. Citado na pág. 17

Fousse et al. (2007) Laurent Fousse, Guillaume Hanrot, Vincent Lefèvre, Patrick Pélissier e Paul Zimmermann. MPFR: A multiple-precision binary floating-point library with correct rounding. ACM Transactions on Mathematical Software, 33(2):13:1-13:15. URL http://doi.acm.org/10. 1145/1236463.1236468. Citado na pág. 10, 26

Hales et al. (2009) Thomas C. Hales, John Harrison, Sean McLaughlin, Tobias Nipkow, Steven Obua e Roland Zumkeller. A revision of the proof of the Kepler conjecture. Discrete and Computational Geometry. Citado na pág. 5

Hansen e Walster (2004) E. R. Hansen e W. G. Walster. Global Optimization Using Interval Analysis. Marcel Dekker and Sun Microsystems, New York, second ed. Citado na pág. 3, 5, 12, 13, $43,60,61$

Hansen e Sengupta (1981) E.R. Hansen e S. Sengupta. Bounding solutions of systems of equations using interval analysis. BIT, 21:203-211. Citado na pág. 5, 65

Issacson e Keller (1994) E. Issacson e H. B. Keller. Analysis of Numerical Methods. Dover, first edition ed. Citado na pág. 64

Kearfott (1996) R. B. Kearfott. Rigorous Global Search: Continuous Problems. Kluwer Academic, Dordrecht, first edition ed. Citado na pág. 3, 5, 10, 11, 12, 13, 43, 46, 60, 61, 85 
Kearfott et al. (2010) R. B. Kearfott, M. T. Nakao, A. Neumaier, S. M. Rump e Hentenryck P. Standardized notation in interval analysis. Computational Technologies, 15(1):7-13. Citado na pág. 5

Kulp et al. (1996) D. Kulp, D. Haussler, M. G. Reese e F. H. Eeckman. A generalized hidden Markov model for the recognition of human genes in DNA. ISMB-96, páginas 134-141. Citado na pág. 2

Levinson et al. (1983) S.E. Levinson, L.R. Rabiner e M.M. Sondhi. An introduction to the application of the theory of probabilistic functions of a Markov process to automatic speech recognition. Bell Syst. Tech. J., 62:1035-1074. ISSN 0005-8580. Citado na pág. 2, 25

Martinez et al. (2004) J.A. Martinez, L.G. Casado, I. García, Ya. D. Sergeyev e B. Toth. On an efficient use of gradient information for accelerating interval global optimization algorithms. Numerical Algorithms, 37:61-69. Citado na pág. 5

Mascarenhas (2014) Walter F. Mascarenhas. The divergence of the bfgs and gauss newton methods. Math. Program., páginas 253-276. Citado na pág. 5

Mascarenhas (1997) WF Mascarenhas. The affine scaling algorithm fails for stepsize 0.999. URL http://unicamp.sibi.usp.br/handle/SBURI/78299. Citado na pág. 5

Moore (1979) R. E. Moore. Methods and Aplications of Interval Analysis. SIAM, Philadelphia, first edition ed. Citado na pág. 2, 3

Moore (1966) R. E. Moore. Interval Arithmetic. Prentice-Hall, Englewood Cliffs, first edition ed. Citado na pág. 5

Moore (1977) R. E. Moore. A test for existence of solutions to nonlinear systems. SIAM Journal of Numerical Analysis, 14(4):611-615. Citado na pág. 5

Moore (1962) R. E. Moore. Interval Arithmetic and Automatic Error Analysis in Digital Computing. Ph.D. dissertation, Department of Mathematics, Stanford University, Stanford, CA, USA. URL http://interval.louisiana.edu/Moores_early_papers/disert.pdf. Also published as Applied Mathematics and Statistics Laboratories Technical Report No. 25. Citado na pág. 5

Moore et al. (2009) Ramon E. Moore, R. Baker Kearfott e Michael J. Cloud. Introduction to Interval Analysis. Society for Industrial and Applied Mathematics, Philadelphia, PA, USA. ISBN 0898716691, 9780898716696. Citado na pág. 8, 61

Neumaier (1990) A. Neumaier. Interval Methods for Systems of Equations. Cambridge Press, Cambridge, first edition ed. Citado na pág. 3

Neumaier (2004) Arnold Neumaier. Complete search in continuous global optimization and constraint satisfaction. Acta Numer., 13:271-369. ISSN 0962-4929. doi: 10.1017/CBO9780511569975. 004. URL http://dx.doi.org/10.1017/CBO9780511569975.004. Citado na pág. 20

Nocedal e Wright (2006) J. Nocedal e J. Wright. Numerical Optimization. Springer, New York, second ed. Citado na pág. $17,44,48,50$

Norris (1998) James R. Norris. Markov chains. Cambridge series in statistical and probabilistic mathematics. Cambridge University Press. ISBN 978-0-521-48181-6. Citado na pág. 1, 17

Nunes et al. (2012) Baltazar Nunes, Isabel Natário e M. Lucília Carvalho. Nowcasting influenza epidemics using non-homogeneous hidden Markov models. Statist. Med., página n/a. doi: 10. 1002/sim.5670. Citado na pág. 2

Pachter e Sturmfels (2007) Lior Pachter e Bernd Sturmfels. The mathematics of phylogenomics. SIAM Rev., 49(1):3-31. ISSN 0036-1445; 1095-7200/e. doi: 10.1137/050632634. Citado na pág. 2 
Pedamallu et al. (2008) C. S. Pedamallu, L. Ozdamar, T. Csendes e T. Vinkó. Efficient interval partitioning for constrained global optimization. Journal of Global Optimization, 42:369-384. Citado na pág. 5,61

Rabiner (1990) Lawrence R. Rabiner. Readings in speech recognition. páginas 267-296. URL http://dl.acm.org/citation.cfm?id=108235.108253. Citado na pág. 2, 17, 18, 23, 25, 47

Rall (1980) L. B. Rall. A comparison of the existence theorems of kantorovich and moore. SIAM Journal of Numerical Analysis, 17(1):148-161. Citado na pág. 5

Rani e Devi (2010) T.Selva Rani e K.Usha Kingsly Devi. Article:isolation of brain tumor segment using hmgmm. International Journal of Computer Applications, 10(9):4-8. Published By Foundation of Computer Science. Citado na pág. 2

Ratz (1996) D. Ratz. Inclusion isotone extended interval arithmetic. Relatório técnico. Citado na pág. 10

Rump (1999) S. M. Rump. INTLAB - INTerval LABoratory, 1999. http://www.ti3.tuharburg.de/rump/. Citado na pág. 15

Rump (2001) S. M. Rump. Rigorous and portable standard functions. BIT, 41(3):540-562. Citado na pág. $10,20,85$

Sahinidis (2014) N.V. Sahinidis. BARON 14.3.1: Global Optimization of Mixed-Integer Nonlinear Programs, 2014. Citado na pág. 4, 17, 67

Sanderson (2010) Conrad Sanderson. Armadillo: An Open Source C ++ Linear Algebra Library for Fast Prototyping and Computationally Intensive Experiments. Relatório técnico, NICTA. Citado na pág. 13

Sunaga (1958) Teruo Sunaga. Theory of an interval algebra and its application to numerical analysis [reprint of res. assoc. appl. geom. mem. 2 (1958), 29?46]. Japan J. Indust. Appl. Math., 26(2-3):125-143. URL http://projecteuclid.org/euclid.jjiam/1317758986. Citado na pág. 5

Tucker (2002) Warwick Tucker. A rigorous ode solver and smale's 14th problem. Foundations of Computational Mathematics, 2(1):53-117. Citado na pág. 5

Votsi et al. (2013) . Votsi, N. Limnios, G. Tsaklidis e E. Papadimitriou. Hidden markov models revealing the stress field underlying the earthquake generation. Physica A: Statistical Mechanics and its Applications, 392(13):2868-2885. Citado na pág. 2

Wright e Kennedy (2000) Kevin Wright e William J. Kennedy. An interval analysis approach to the em algorithm. Journal of Computational and Graphical Statistics, 9(2):pp. 303-318. ISSN 10618600. URL http://www.jstor.org/stable/1390656. Citado na pág. 4

Wächter e Biegler (2006) Andreas Wächter e Lorenz T. Biegler. On the implementation of an interior-point filter line-search algorithm for large-scale nonlinear programming. Mathematical Programming, 106(1):25-57. Citado na pág. 17, 50

Žilinskas e Žilinskas (2010) Antanas Žilinskas e Julius Žilinskas. Interval arithmetic based optimization in nonlinear regression. Informatica (Vilnius), 21(1):149-158. ISSN 0868-4952. Citado na pág. 4

Zucchini e MacDonald (2009) Walter Zucchini e Iain L. MacDonald. Hidden Markov models for time series : an introduction using $R$. Monographs on statistics and applied probability. CRC Press, Boca Raton. ISBN 978-1-584-88573-3. Citado na pág. 1, 2, 15, 18, 25 


\section{Índice Remissivo}

BARON, 4, 17, 44, 67, 84, 93

$\mathrm{C}++, 4,10,27,50$

Cadeia de Markov, 1, 15, 17-19, 23, 43, 67

Forward-Backward, 24

frexp, 27, 93

Interval Branch and Bound, 3-5, 13, 19, 43, 44, $46,52,60-67,85,92,93$

INTLAB, 11

ldexp, 27, 93

Método

De Pontos Interiores, 50-52, 56, 59, 93

De Gauss-Seidel, 5, 10, 12, 13, 15, 20, 21, 65, 93

De Newton Intervalar, 12, 65, 66

Modelo de Mistura, 1

Modelos Ocultos de Makorv, 1

Propagação

Da Função Objetivo, 65-67

De Restrições, 20, 62

Recursão Backward, 23-26, 29-32, 37, 46, 63

Recursão forward, 24

Teorema Fundamental da Aritmética Intervalar, 8,12

verifylss, 15

ZBMath, 2 